\title{
Rotating higher spin partition functions and extended BMS symmetries
}

\author{
A. Campoleoni, ${ }^{a, 1}$ H.A. Gonzalez, ${ }^{a}$ B. Oblak ${ }^{a, b, 2}$ and M. Riegler ${ }^{c}$ \\ ${ }^{a}$ Université Libre de Bruxelles and International Solvay Institutes, \\ ULB-Campus Plaine CP231, 1050 Brussels, Belgium \\ ${ }^{b}$ DAMTP, Centre for Mathematical Sciences, University of Cambridge, \\ Wilberforce Road, Cambridge CB3 OWA, U.K. \\ ${ }^{c}$ Institute for Theoretical Physics, Vienna University of Technology, \\ Wiedner Hauptstrasse 8-10, A-1040 Vienna, Austria \\ E-mail: andrea.campoleoni@ulb.ac.be, hgonzale@ulb.ac.be, \\ boblak@ulb.ac.be, rieglerm@hep.itp.tuwien.ac.at
}

ABSTRACT: We evaluate one-loop partition functions of higher-spin fields in thermal flat space with angular potentials; this computation is performed in arbitrary space-time dimension, and the result is a simple combination of Poincare characters. We then focus on dimension three, showing that suitable products of one-loop partition functions coincide with vacuum characters of higher-spin asymptotic symmetry algebras at null infinity. These are extensions of the $\mathfrak{b m s}_{3}$ algebra that emerges in pure gravity, and we propose a way to build their unitary representations and to compute the associated characters. We also extend our investigations to supergravity and to a class of gauge theories involving higher-spin fermionic fields.

KeYwords: Field Theories in Higher Dimensions, Field Theories in Lower Dimensions, Higher Spin Gravity, Higher Spin Symmetry

ARXIV EPRINT: 1512.03353

\footnotetext{
${ }^{1}$ Postdoctoral Researcher of the Fund for Scientific Research-FNRS Belgium.

${ }^{2}$ Research fellow of the Fund for Scientific Research-FNRS Belgium.
} 


\section{Contents}

1 Introduction 1

2 Partition functions in flat space 4

2.1 Heat kernels and the method of images 4

$\begin{array}{lr}2.2 & \text { Bosonic higher spins }\end{array}$

$\begin{array}{lll}2.3 & \text { Fermionic higher spins } & 11\end{array}$

$\begin{array}{lll}2.4 & \text { Relation to Poincaré characters } & 14\end{array}$

3 Three-dimensional applications $\quad 18$

$\begin{array}{lll}3.1 & \mathrm{BMS}_{3} \text { particles and induced } \mathfrak{b m s}_{3} \text { modules } & 18\end{array}$

$\begin{array}{lll}3.2 & \text { Characters of flat } \mathcal{W}_{N} \text { algebras } & 22\end{array}$

$\begin{array}{lll}3.3 & \text { Supersymmetry and super } \mathrm{BMS}_{3} \text { characters } & 30\end{array}$

4 Further directions $\quad 36$

A From mixed traces to bosonic characters $\quad 38$

$\begin{array}{lll}\text { A.1 Mixed traces and symmetric polynomials } & 38\end{array}$

A.2 Symmetric polynomials and $\mathrm{SO}(D)$ characters 41

A.3 Differences of $\mathrm{SO}(D)$ characters 42

A.4 From $\mathrm{SO}(D)$ to $\mathrm{SO}(D-1)$

B From mixed traces to fermionic characters $\quad 45$

B.1 Mixed traces and symmetric polynomials $\quad 45$

B.2 Symmetric polynomials and $\mathrm{SO}(D)$ characters 46

\section{Introduction}

The structure of the known interacting field theories involving particles of spin greater than two depends significantly on the presence or absence of a cosmological constant. On (anti) de Sitter backgrounds of any dimension, Vasiliev's equations describe higher-spin gauge theories with an infinite tower of massless fields of increasing spin [1]. These models display several unconventional features, mainly because interactions involve more than two derivatives. In flat space the situation is even subtler, since one loses the option to balance higher derivatives by inverse powers of the cosmological constant. As a result, interactions of massless fields in flat space are expected to be fraught with more severe non-localities than their (A)dS peers (see e.g. [2] for a review), and it is not yet clear if consistent interacting theories can be defined at all. These difficulties, however, are absent for massive fields, which bring in a dimensionful parameter that can play a role analogous 
to that of the cosmological constant. String field theories indeed involve infinitely many massive higher-spin fields and they can be defined on flat backgrounds.

Therefore, even if (A)dS backgrounds favour interactions of massless higher-spin particles, flat space is not completely ruled out by higher spins. Several indications also suggest that string models could actually be broken phases of a higher-spin gauge theory (see e.g. [3] for a recent review). In order to clarify this issue one should understand if the striking differences in higher-spin theories with or without cosmological constant are really fundamental, or if they are induced by technical assumptions on the "allowed" field theories. At present we indeed control higher-spin gauge theories only in a context in which we do not fully control String Theory. To make progress in this quest, it is important to develop tools to analyse the elusive higher-spin theories in flat space and to study the pathologies of the flat limit of the known interacting theories in (A)dS.

In this paper we consolidate one of these tools for flat space; we compute one-loop partition functions for higher-spin fields in $D$-dimensional Minkowski space at finite temperature and with non-vanishing angular potentials. Although they are determined by the free theory, one-loop partition functions often provide useful information on the consistency of a given spectrum for a possible interacting quantum field theory. This powerful feature has been extensively exploited for higher-spin gauge theories on AdS backgrounds: in $D=3$ the comparison between bulk and boundary partition functions [4-6] has been an important ingredient in defining the holographic correspondence between higher-spin gauge theories and minimal model CFTs [7]. In $D>3$ the analysis of one-loop partition functions of infinite sets of higher-spin fields provided the first quantum checks [8-12] of analogous AdS/CFT dualities [13]. More recently, holographic considerations driven by the structure of one-loop partition functions have also been used to conjecture the existence of consistent quantum higher-spin gauge theories in AdS with spectra that differ from those of Vasiliev's theories [14-16].

In flat space this tool has been poorly employed in the higher-spin context; aside from computing one-loop partition functions for any $D$, here we also provide a first application of our results when $D=3$. This is a promising setup to explore relations between higher-spin theories in flat and (A)dS spaces because, in contrast with what happens when $D>3$, the limit of vanishing cosmological constant does not entail any subtlety. The main reason is that, in the absence of matter couplings, higher-spin gauge theories in $\mathrm{AdS}_{3}$ are described by Chern-Simons actions [17] that can be cast in the form

$$
S=\frac{k_{\mathfrak{g}}}{16 \pi G} \int \operatorname{tr}\left(e \wedge R+\frac{1}{3 \ell^{2}} e \wedge e \wedge e\right), \quad \text { with } R=d \omega+\omega \wedge \omega,
$$

where $e$ and $\omega$ are one-forms that generalise the gravity vielbein and spin connection and that take values in a suitable gauge algebra (typically $\mathfrak{s l}(N, \mathbb{R})$ for theories involving fields of spin $2,3, \ldots, N$ - see e.g. [18] for a review). In (1.1) $G$ denotes Newton's constant and $k_{\mathfrak{g}}$ is a factor that depends on the normalisation of the trace, while $\ell$ denotes the AdS radius. One can clearly consider the limit $\ell \rightarrow \infty$ in the action. ${ }^{1}$ This simplification is related to the absence of local degrees of freedom in theories involving fields of spin $s \geq 2$. Another

\footnotetext{
${ }^{1}$ In the metric-like formulation of the dynamics [19, 20], on which we rely to compute partition functions,
} 
key feature of gravitational theories in three dimensions is the richness of their asymptotic symmetries in both AdS [22-26] and flat space [27-32]. The combination of simplicity and powerful infinite-dimensional asymptotic symmetries makes these models important testing grounds for the holographic principle, in both its AdS/CFT realisation and its possible flat space counterpart. However, in spite of the straightforward way one can obtain interacting actions in flat space from the $\ell \rightarrow \infty$ limit of (1.1), higher-spin gauge theories in flat space are arguably less understood than those in $\mathrm{AdS}_{3}$. An important reason is that their asymptotic symmetry algebras at null infinity are less familiar than those that emerge in $\mathrm{AdS}_{3}$ at spatial infinity. The latter are typically $\mathcal{W}_{N}$ algebras, which are well studied global symmetries in two-dimensional CFT [33]. In the following we propose a characterisation of the unitary representations of their flat space counterparts - that we call "flat $\mathcal{W}_{N}$ algebras" - and we test our proposal by matching their vacuum characters with suitable products of partition functions of higher-spin fields. In this process we thus achieve two goals: on the one hand we improve the current understanding of the representation theory of flat $\mathcal{W}_{N}$ algebras. On the other hand, in analogy with similar results in $\operatorname{AdS}_{3}[4,6,34]$, we confirm that the asymptotic symmetries identified by the classical analysis of [30-32] are a robust feature of these models, that should persist also at the quantum level.

The paper is organised as follows: in section 2 we compute one-loop partition functions of higher-spin fields on Minkowski space of arbitrary dimensions $D$ with finite temperature $1 / \beta$ and a maximal number of angular potentials $\vec{\theta}$. We employ the heat kernel method of [34]. As already shown for gravity in $D=3$ [35], these techniques are more tractable in flat space than in (A)dS, so that we do not have to resort to their successive refinements [36, 37]. In section 2.2 we study massive and massless bosonic fields of any (discrete) spin, whose partition functions are given in $(2.24)$ and $(2.28,2.30)$. In section 2.3 we then move to massive and massless fermionic fields of any spin, whose partition functions are given in (2.49). In section 2.4 we rewrite partition functions in terms of characters of the Poincaré group. For massive fields we obtain

$$
Z_{M, s}[\beta, \vec{\theta}]=\exp \left[\sum_{n=1}^{\infty} \frac{1}{n} \chi_{M, s}[n \vec{\theta}, i n \beta]\right],
$$

where $\chi_{M, s}$ is a character of a representation of the Poincaré group of mass $M$ and spin $s$. For massless fields this natural rewriting has to be amended when $D$ is odd, as one also has to introduce suitable angle-dependent coefficients.

In section 3 we focus on $D=3$. In section 3.1 we begin by reviewing several aspects of the representation theory of the $\mathrm{BMS}_{3}$ group - i.e. of the group of asymptotic symmetries at null infinity of pure gravity in $D=3$ - that are relevant in the following. We emphasise that its representations are induced representations classified by orbits of supermomenta, and we show how one can describe the representations of the corresponding $\mathfrak{b m s}_{3}$ algebra in terms of induced modules. In section 3.2 we move to higher spins, proposing to build unitary representations of flat $\mathcal{W}_{N}$ algebras as Hilbert spaces of wavefunctions defined on

the limit is well defined because interactions involve at most two derivatives in $D=3$, so that no inverse powers of the cosmological constant enter the action [21]. 
coadjoint $\mathcal{W}_{N}$ orbits of (higher-spin) supermomenta. We also compute vacuum characters and the characters of other illustrative representations. We then test our proposal by checking that vacuum characters of flat $\mathcal{W}_{N}$ algebras - which take the form

$$
\chi_{\mathrm{vac}}[\theta, \beta]=e^{\frac{\beta}{8 G}} \prod_{s=2}^{N}\left(\prod_{n=s}^{\infty} \frac{1}{\mid 1-e^{\left.i n(\theta+i \epsilon)\right|^{2}}}\right),
$$

where $\epsilon$ is a regulator that ensures the convergence of the infinite product - match the product of the partition functions of fields of spin $2,3, \ldots, N$ computed in section 2.2. We also take advantage of the description of these representations in terms of induced modules to make contact with previous proposals on the structure of representations of flat $\mathcal{W}_{N}$ algebras. This allows us to explain how our representations evade some no-go arguments against the existence of unitary higher-spin gauge theories in three-dimensional flat space that appeared in the literature [38]. In section 3.3 we include fermions: we discuss in detail the representation theory of the $\mathcal{N}=1$ extension of the $\mathrm{BMS}_{3}$ group relevant for supergravity. We then extend our results to hypergravity theories describing the gravitational coupling of a massless field of spin $s+1 / 2$. In both cases we also exhibit the matching between vacuum characters and the product of partition functions of fields of spin 2 and $s+1 / 2$.

We close the paper with a discussion of possible extensions and applications of our work even beyond three dimensions (section 4), while two technical appendices fill the gap between the results of heat kernel computations and the rewriting of partitions functions in terms of Poincaré characters.

\section{Partition functions in flat space}

We wish to study one-loop partition functions of higher-spin fields living in $D$-dimensional Minkowski space at finite temperature $1 / \beta$, and with non-zero angular potentials. We will denote these potentials as $\vec{\theta}=\left(\theta_{1}, \ldots, \theta_{r}\right)$, where $r=\lfloor(D-1) / 2\rfloor$ is the rank of $\operatorname{SO}(D-1)$, that is, the maximal number of independent rotations in $(D-1)$ space dimensions. The computation involves a functional integral over fields living on a quotient of $\mathbb{R}^{D}$, where the easiest way to incorporate one-loop effects is the heat kernel method. Accordingly, we will now briefly review this approach, before analysing separately bosons (section 2.2) and fermions (section 2.3). In section 2.4 we then rewrite partition functions in terms of characters of the Poincaré group.

\subsection{Heat kernels and the method of images}

Our goal is to compute partition functions of the form

$$
Z[\beta, \vec{\theta}]=\int \mathcal{D} \phi e^{-S[\phi]}
$$

where $\phi$ is some collection of fields (bosonic or fermionic) defined on a thermal quotient $\mathbb{R}^{D} / \mathbb{Z}$ of flat Euclidean space, satisfying suitable (anti)periodicity conditions. (The explicit action of $\mathbb{Z}$ on $\mathbb{R}^{D}$, with its dependence on $\beta$ and $\vec{\theta}$, will be displayed below — see eq. (2.7).) 
The functional $S[\phi]$ is a Euclidean action for these fields. Expression (2.1) can be evaluated perturbatively around a saddle point $\phi_{c}$ of $S$, leading to the semi-classical (one-loop) result

$$
Z[\beta, \vec{\theta}] \sim e^{-S\left[\phi_{c}\right]}\left[\left.\operatorname{det}\left(\frac{\delta^{2} S}{\delta \phi \delta \phi}\right)\right|_{\phi_{c}}\right]^{\#}
$$

where the exponent \# depends on the nature of the fields that were integrated out. The quantity $\delta^{2} S / \delta \phi(x) \delta \phi(y)$ appearing in this expression is a differential operator acting on sections of a suitable vector bundle over $\mathbb{R}^{D} / \mathbb{Z}$. The evaluation of the one-loop contribution to the partition function thus boils down to that of a functional determinant.

The heat kernel method is a neat way to compute such determinants; after gaugefixing, they reduce to determinants of operators of the form $\left(-\Delta+M^{2}\right)$. In short (see e.g. [34, 39] for details), it allows one to express $\operatorname{det}\left(-\Delta+M^{2}\right)$ as an integral

$$
-\log \operatorname{det}\left(-\Delta+M^{2}\right)=\int_{0}^{\infty} \frac{d t}{t} \int d^{D} x \operatorname{Tr}[K(t, x, x)]
$$

up to an ultraviolet divergence that can be regulated with standard methods. Here $K\left(t, x, x^{\prime}\right)$ is a matrix-valued bitensor known as the heat kernel associated with $\left(-\Delta+M^{2}\right)$. It satisfies the heat equation

$$
\frac{\partial}{\partial t} K\left(t, x, x^{\prime}\right)-\left(\Delta_{x}-M^{2}\right) K\left(t, x, x^{\prime}\right)=0,
$$

along with the initial condition

$$
K\left(t=0, x, x^{\prime}\right)=\delta^{(D)}\left(x-x^{\prime}\right) \mathbb{I},
$$

with $\mathbb{I}$ the identity matrix having the same tensor structure as $K$ (here omitted for brevity).

Heat kernels are well suited for the computation of functional determinants on quotient spaces. Indeed, suppose $\Gamma$ is a discrete subgroup of the isometry group of $\mathbb{R}^{D}$, acting freely on $\mathbb{R}^{D}$. Introducing the equivalence relation $x \sim y$ if there exists a $\gamma \in \Gamma$ such that $\gamma(x)=y$, we define the quotient manifold $\mathbb{R}^{D} / \Gamma$ as the set of corresponding equivalence classes. Given a differential operator $\Delta$ on $\mathbb{R}^{D}$, it naturally induces a differential operator on $\mathbb{R}^{D} / \Gamma$, acting on fields that satisfy suitable (anti)periodicity conditions. Because the heat equation (2.4) is linear, the heat kernel on the quotient space can be obtained from the heat kernel on $\mathbb{R}^{D}$ by the method of images:

$$
K^{\mathbb{R}^{D} / \Gamma}\left(t, x, x^{\prime}\right)=\sum_{\gamma \in \Gamma} K\left(t, x, \gamma\left(x^{\prime}\right)\right) .
$$

Here, abusing notation slightly, $x$ and $x^{\prime}$ denote points both in $\mathbb{R}^{D}$ and in its quotient. In writing (2.6) we are assuming, for simplicity, that the tensor structure of $K$ is trivial, but as soon as $K$ carries tensor or spinor indices (i.e. whenever the fields under consideration have non-zero spin), the right-hand side involves Jacobians accounting for the non-trivial transformation law of $K$.

We will be concerned with thermal quantum field theories on rotating Minkowski space. This means we will define our fields on a quotient $\mathbb{R}^{D} / \mathbb{Z}$ of Euclidean space, with 
the action of $\mathbb{Z}$ defined as follows. For odd $D$, we endow $\mathbb{R}^{D}$ with Cartesian coordinates $\left(x_{i}, y_{i}\right)$ (where $\left.i=1, \ldots, r\right)$ and a Euclidean time coordinate $\tau$, so that an integer $n \in \mathbb{Z}$ acts on $\mathbb{R}^{D}$ according to

$$
\gamma^{n}\left(\begin{array}{l}
x_{i} \\
y_{i}
\end{array}\right)=\left(\begin{array}{cc}
\cos \left(n \theta_{i}\right) & -\sin \left(n \theta_{i}\right) \\
\sin \left(n \theta_{i}\right) & \cos \left(n \theta_{i}\right)
\end{array}\right) \cdot\left(\begin{array}{l}
x_{i} \\
y_{i}
\end{array}\right) \equiv R\left(n \theta_{i}\right) \cdot\left(\begin{array}{l}
x_{i} \\
y_{i}
\end{array}\right), \quad \gamma^{n}(\tau)=\tau+n \beta .
$$

For even $D$ we simply add one more spatial coordinate $z$, invariant under $\mathbb{Z}$. In terms of the coordinates $\left\{x_{1}, y_{1}, \ldots, x_{r}, y_{r}, \tau\right\}$ (and possibly $z$ at the end of this list for even $D$ ), the Lorentz matrix implementing the rotation $(2.7)$ is the $n^{\text {th }}$ power of

$$
J=\left(\begin{array}{cccc}
R\left(\theta_{1}\right) & 0 & \cdots & 0 \\
0 & \ddots & 0 & \vdots \\
\vdots & 0 & R\left(\theta_{r}\right) & 0 \\
0 & \cdots & 0 & 1
\end{array}\right) \quad \text { or } \quad\left(\begin{array}{ccccc}
R\left(\theta_{1}\right) & 0 & \cdots & 0 & 0 \\
0 & \ddots & 0 & \vdots & 0 \\
\vdots & 0 & R\left(\theta_{r}\right) & 0 & 0 \\
0 & \cdots & 0 & 1 & 0 \\
0 & \cdots & 0 & 0 & 1
\end{array}\right)
$$

for $D$ odd or $D$ even, respectively. Being isometries of flat space, these transformations are linear maps in Cartesian coordinates, and their $n^{\text {th }}$ power therefore coincides with the Jacobian matrix $\partial \gamma^{n}(x)^{\mu} / \partial x^{\nu}$ that will be needed later for the method of images. Throughout this paper we take all angles $\theta_{1}, \ldots, \theta_{r}$ to be non-vanishing. We now display the computation of one-loop partition functions on $\mathbb{R}^{D} / \mathbb{Z}$, first for bosonic, then for fermionic higher-spin fields.

\subsection{Bosonic higher spins}

In this subsection we study the rotating one-loop partition function of a single bosonic field with spin $s$ and mass $M$ (including the massless case). For $M>0$ its Euclidean free action can be presented either (i) using a symmetric traceless field $\phi_{\mu_{1} \ldots \mu_{s}}$ of rank $s$ and a tower of auxiliary fields of ranks $s-2, s-3, \ldots, 0$ that do not display any gauge symmetry [40], or (ii) using a set of doubly-traceless fields of ranks $s, s-1, \ldots, 0$ subject to a gauge symmetry generated by traceless gauge parameters of ranks $s-1, s-2, \ldots, 0$ [41]. In the latter case, the quadratic action is given by the sum of Fronsdal actions [19] for each of the involved fields, plus a set of cross-coupling terms with one derivative proportional to $M$ and a set of terms without derivatives proportional to $M^{2}$. In the massless limit, all couplings vanish and one can consider independently the Fronsdal action for the field of highest rank:

$$
S\left[\phi_{\mu_{1} \ldots \mu_{s}}\right]=\frac{1}{2} \int d^{D} x \phi^{\mu_{1} \ldots \mu_{s}}\left(\mathcal{F}_{\mu_{1} \ldots \mu_{s}}-\frac{1}{2} \delta_{\left(\mu_{1} \mu_{2}\right.} \mathcal{F}_{\left.\mu_{3} \ldots \mu_{s}\right) \lambda}{ }^{\lambda}\right),
$$

where

$$
\mathcal{F}_{\mu_{1} \ldots \mu_{s}}=\square \phi_{\mu_{1} \ldots \mu_{s}}-\partial_{\left(\mu_{1} \mid\right.} \partial^{\lambda} \phi_{\left.\mid \mu_{2} \ldots \mu_{s}\right) \lambda}+\partial_{\left(\mu_{1}\right.} \partial_{\mu_{2}} \phi_{\left.\mu_{3} \ldots \mu_{s}\right) \lambda}{ }^{\lambda}
$$

and parentheses denote the symmetrisation of the indices they enclose, with the minimum number of terms needed and without any overall factor. In the alternative formulation of the dynamics where no gauge symmetry is present [40], in the massless limit all auxiliary 
fields except the one of rank $s-2$ decouple. The remaining fields can be combined into a doubly traceless field whose action is given again by (2.9) [19]. For further details we refer e.g. to [42].

Note that in all space-time dimensions other than three, the vacuum saddle point of the action (2.9) (or of its massive counterpart) is the trivial field configuration $\phi_{\mu_{1} \ldots \mu_{s}}=0$. Accordingly, the whole partition function (2.2) is captured by its one-loop piece. In $D=3$, the presence of a mass gap makes this situation slightly different; we will return to this issue at the end of this subsection.

Massive case. Applying e.g. the techniques of [4] to the presentation of the Euclidean action of a massive field of spin $s$ of [41], one finds that the partition function is given by

$$
\log Z=-\frac{1}{2} \log \operatorname{det}\left(-\Delta^{(s)}+M^{2}\right)+\frac{1}{2} \log \operatorname{det}\left(-\Delta^{(s-1)}+M^{2}\right),
$$

where $\Delta^{(s)}$ is the Laplacian $\partial_{\mu} \partial^{\mu}$ acting on periodic, ${ }^{2}$ symmetric, traceless tensor fields with $s$ indices on $\mathbb{R}^{D} / \mathbb{Z}$. We will denote the heat kernel associated with $\left(-\Delta^{(s)}+M^{2}\right)$ on $\mathbb{R}^{D}$ as $K_{\mu_{s}, \nu_{s}}\left(t, x, x^{\prime}\right)$, where $\mu_{s}$ and $\nu_{s}$ are shorthands that denote sets of $s$ symmetrised indices. The heat equation (2.4) and initial condition (2.5) for $K_{\mu_{s}, \nu_{s}}\left(t, x, x^{\prime}\right)$ then read

$$
\left(\Delta^{(s)}-M^{2}-\partial_{t}\right) K_{\mu_{s}, \nu_{s}}=0, \quad K_{\mu_{s}, \nu_{s}}\left(t=0, x, x^{\prime}\right)=\mathbb{I}_{\mu_{s}, \nu_{s}} \delta^{(D)}\left(x-x^{\prime}\right),
$$

where $\mathbb{I}_{\mu_{s}, \nu_{s}}$ is an identity matrix with the same tensor structure as $K_{\mu_{s}, \nu_{s}}$. A set of repeated covariant or contravariant indices also denotes a set of indices that are symmetrised with the minimum number of terms required and without multiplicative factors, while contractions involve as usual a covariant and a contravariant index. The tracelessness condition on the heat kernel amounts e.g. to

$$
\delta^{\mu \mu} K_{\mu_{s}, \nu_{s}}=\delta^{\nu \nu} K_{\mu_{s}, \nu_{s}}=0 .
$$

The solution of (2.12) fulfilling this condition is

$$
K_{\mu_{s}, \nu_{s}}\left(t, x, x^{\prime}\right)=\frac{1}{(4 \pi t)^{D / 2}} e^{-M^{2} t-\frac{1}{4 t}\left|x-x^{\prime}\right|^{2}} \mathbb{I}_{\mu_{s}, \nu_{s}},
$$

with

$$
\mathbb{I}_{\mu_{s}, \nu_{s}}=\sum_{n=0}^{\left\lfloor\frac{s}{2}\right\rfloor} \frac{(-1)^{n} 2^{n} n ![D+2(s-n-2)] ! !}{s ![D+2(s-2)] ! !} \delta_{\mu \mu}^{n} \delta_{\mu \nu}^{s-2 n} \delta_{\nu \nu}^{n} .
$$

Note that the dependence of this heat kernel on the space-time points $x, x^{\prime}$ and on Schwinger proper time $t$ is that of a scalar heat kernel, and completely factorises from its spin/index structure which is wholly accounted for by the matrix $\mathbb{I}$. This simplification is the main reason why explicit heat kernel computations are more tractable in flat space than in AdS or dS.

To determine the heat kernel associated with the operator $\left(-\Delta^{(s)}+M^{2}\right)$ on $\mathbb{R}^{D} / \mathbb{Z}$, we use the method of images (2.6), taking care of the non-trivial index structure. Denoting

\footnotetext{
${ }^{2}$ More precisely, the field at Euclidean time $\tau+\beta$ is rotated by $\vec{\theta}$ with respect to the field at time $\tau$.
} 
the matrix (2.8) by $J_{\alpha}{ }^{\beta}$ (it is the Jacobian of the transformation $x \mapsto \gamma(x)$ ), the spin- $s$ heat kernel on $\mathbb{R}^{D} / \mathbb{Z}$ is

$$
K_{\mu_{s}, \nu_{s}}^{\mathbb{R}^{D} / \mathbb{Z}}\left(t, x, x^{\prime}\right)=\sum_{n \in \mathbb{Z}}\left(J^{n}\right)_{\alpha}^{\beta} \ldots\left(J^{n}\right)_{\alpha}^{\beta} K_{\mu_{s}, \beta_{s}}\left(t, x, \gamma^{n}\left(x^{\prime}\right)\right)
$$

where we recall again that repeated covariant or contravariant indices are meant to be symmetrised with the minimum number of terms required and without multiplicative factors, while repeating a covariant index in a contravariant position denotes a contraction. Accordingly, formula (2.3) gives the determinant of $\left(-\Delta^{(s)}+M^{2}\right)$ on $\mathbb{R}^{D} / \mathbb{Z}$ as

$$
\begin{aligned}
& -\log \operatorname{det}\left(-\Delta^{(s)}+M^{2}\right)=\int_{0}^{+\infty} \frac{d t}{t} \int_{\mathbb{R}^{D} / \mathbb{Z}} d^{D} x\left(\delta^{\mu \alpha}\right)^{s} K_{\mu_{s}, \alpha_{s}}^{\mathbb{R}^{D} / \mathbb{Z}}(t, x, x) \\
& =\sum_{n \in \mathbb{Z}}\left(J^{n}\right)^{\mu \beta} \cdots\left(J^{n}\right)^{\mu \beta} \mathbb{I}_{\mu_{s}, \beta_{s}} \int_{0}^{+\infty} \frac{d t}{t} \int_{\mathbb{R}^{D} / \mathbb{Z}} d^{D} x \frac{1}{(4 \pi t)^{D / 2}} e^{-M^{2} t-\frac{1}{4 t}\left|x-\gamma^{n}(x)\right|^{2}} .
\end{aligned}
$$

In this series the term $n=0$ contains both an ultraviolet divergence (due to the singular behaviour of the integrand as $t \rightarrow 0$ ) and an infrared one (due to the integral of a constant over $\mathbb{R}^{D} / \mathbb{Z}$ ), proportional to the product $\beta V$ with $V$ the spatial volume of the system. This divergence is a quantum contribution to the vacuum energy, which we ignore from now on. The only non-trivial one-loop contribution we must take into account then comes from the terms $n \neq 0$ in (2.17). Using

$$
\left|x-\gamma^{n}(x)\right|^{2}=n^{2} \beta^{2}+\sum_{i=1}^{r} 4 \sin ^{2}\left(n \theta_{i} / 2\right)\left(x_{i}^{2}+y_{i}^{2}\right)
$$

in terms of the coordinates introduced around (2.7), the integrals over $t$ and $x$ give

$$
-\log \operatorname{det}\left(-\Delta^{(s)}+M^{2}\right)=\sum_{n \in \mathbb{Z}^{*}} \frac{1}{|n|} \frac{\chi_{s}[n \vec{\theta}, \vec{\epsilon}]}{\prod_{j=1}^{r} \mid 1-e^{\left.i n\left(\theta_{j}+i \epsilon_{j}\right)\right|^{2}}} \times \begin{cases}e^{-|n| \beta M} & \text { if } D \text { odd } \\ \frac{M \Delta z}{\pi} K_{1}(|n| \beta M) & \text { if } D \text { even }\end{cases}
$$

where $K_{1}$ is the first modified Bessel function of the second kind, and $\Delta z \equiv \int_{-\infty}^{+\infty} d z$ is an infrared divergence that arises in even dimensions because the $z$ axis is left fixed by the rotation (2.8). Following [35] we have added small imaginary parts to the angles $\theta_{j}$ to make the series convergent. Similarly

$$
\chi_{s}[n \vec{\theta}, \vec{\epsilon}] \equiv\left(J^{n}\right)^{\mu \beta} \ldots\left(J^{n}\right)^{\mu \beta} \mathbb{I}_{\mu_{s}, \beta_{s}} \equiv\left[\left(J^{n}\right)^{\mu \beta}\right]^{s} \mathbb{I}_{\mu_{s}, \beta_{s}}
$$

is the full mixed trace of $\mathbb{I}_{\mu_{s}, \nu_{s}}$, with the understanding that $\theta_{j}$ is replaced by $\theta_{j} \pm i \epsilon_{j}$ in all positive powers of $e^{ \pm i \theta_{j}}$. For odd $D$, the result of this regularisation agrees with the flat limit of the AdS one-loop determinant, in which case the parameters $\epsilon_{j}=\beta / \ell$ are remnants of the inverse temperature (with $\ell$ the AdS radius). ${ }^{3}$ For even $D$, the flat limit of the AdS result contains an infrared divergence; it is not obvious to us how this divergence

\footnotetext{
${ }^{3}$ According to [43], a different regularisation may produce a completely different, albeit finite, result.
} 
can be regularised so as to reproduce the combination $\Delta z \cdot K_{1}$ of $(2.19)$, but apart from this subtlety, the other terms of the expression indeed coincide with the flat limit of their AdS counterparts. From now on we will often omit displaying the $\epsilon$-regularisation explicitly, keeping it only in the final results.

Expression (2.19) is a higher-dimensional, higher-spin extension of the result derived previously for spin two in three dimensions in [35]. In particular, the divergence as $\epsilon_{j} \rightarrow 0$ is the same as in three dimensions. The new ingredient is the angle-dependent trace (2.20); in appendices A.1 and A.2 we show that it is the character of an irreducible, unitary representation of $\mathrm{SO}(D)$ with highest weight $\lambda_{s} \equiv(s, 0, \ldots, 0)$. More precisely, let $H_{i}$ denote the generator of rotations in the plane $\left(x_{i}, y_{i}\right)$, in the coordinates defined around (2.7). Then the Cartan subalgebra $\mathfrak{h}$ of $\mathfrak{s o}(D)$ is generated by $H_{1}, \ldots, H_{r}$, plus, if $D$ is even, a generator of rotations in the plane $(\tau, z)$. Denoting the dual basis of $\mathfrak{h}^{*}$ by $L_{1}, \ldots, L_{r}$ (plus possibly $L_{r+1}$ if $D$ is even), we can consider the weight $\lambda_{s}=s L_{1}$ whose only non-zero component (in the basis of $L_{i}$ 's) is the first one. The character of the corresponding highest-weight representation of $\mathfrak{s o}(D)$ coincides with expression (A.1):

$$
\chi_{s}[n \vec{\theta}]=\chi_{\lambda_{s}}\left[n \theta_{1}, \ldots, n \theta_{r}\right] \quad \text { or } \quad \chi_{\lambda_{s}}\left[n \theta_{1}, \ldots, n \theta_{r}, 0\right],
$$

for $D$ odd or even, respectively.

We can finally display an explicit formula for the one-loop partition function (2.11). Using expression (2.19) for the one-loop determinant together with property (2.21), we find

$$
Z[\beta, \vec{\theta}]=\exp \left[\sum_{n=1}^{\infty} \frac{n^{-1}}{\prod_{j=1}^{r}\left|1-e^{i n \theta_{j}}\right|^{2}} \times\left\{\begin{array}{l}
\left(\chi_{\lambda_{s}}^{\mathrm{SO}(D)}[n \vec{\theta}]-\chi_{\lambda_{s-1}}^{\mathrm{SO}(D)}[n \vec{\theta}]\right) e^{-n \beta M} \\
\left(\chi_{\lambda_{s}}^{\mathrm{SO}(D)}[n \vec{\theta}, 0]-\chi_{\lambda_{s-1}}^{\mathrm{SO}(D)}[n \vec{\theta}, 0]\right) \frac{M \Delta z}{\pi} K_{1}(n \beta M)
\end{array}\right],\right.
$$

where the upper (resp. lower) line corresponds to the case where $D$ is odd (resp. even). Remarkably, the differences of $\mathrm{SO}(D)$ characters appearing here can be simplified: according to eqs. (A.26a) and (A.27), the difference of two $\mathrm{SO}(D)$ characters with weights $(s, 0, \ldots, 0)$ and $(s-1,0, \ldots, 0)$ is a (sum of) character(s) of $\mathrm{SO}(D-1)$ :

$$
\left.\begin{array}{ll}
\chi_{\lambda_{s}}^{\mathrm{SO}(D)}[\vec{\theta}]-\chi_{\lambda_{s-1}}^{\mathrm{SO}(D)}[\vec{\theta}] & (\mathrm{D} \text { odd }) \\
\chi_{\lambda_{s}}^{\mathrm{SO}(D)}[\vec{\theta}, 0]-\chi_{\lambda_{s-1}}^{\mathrm{SO}(D)}[\vec{\theta}, 0] & \text { (D even) }
\end{array}\right\}=\chi_{\lambda_{s}}^{\mathrm{SO}(D-1)}[\vec{\theta}] .
$$

Since $r=\lfloor(D-1) / 2\rfloor$ is the rank of $\mathrm{SO}(D-1)$, the right-hand side of this equality makes sense regardless of the parity of $D$, and the partition function (2.22) boils down to

$$
Z[\beta, \vec{\theta}]=\exp \left[\sum_{n=1}^{\infty} \frac{1}{n} \frac{\chi_{\lambda_{s}}^{\mathrm{SO}(D-1)}[n \vec{\theta}, \vec{\epsilon}]}{\prod_{j=1}^{r}\left|1-e^{i n\left(\theta_{j}+i \epsilon_{j}\right)}\right|^{2}} \times\left\{\begin{array}{ll}
e^{-n \beta M} & (D \text { odd }) \\
\frac{M \Delta z}{\pi} K_{1}(n \beta M) & (D \text { even })
\end{array}\right] .\right.
$$

In section 2.4 we will show that the function of $n \vec{\theta}$ and $n \beta$ appearing here in the sum over $n$ is in fact the character of an irreducible, unitary representation of the Poincaré group with mass $M$ and spin $s$ (see eq. (2.55)). A similar result holds in Anti-De Sitter space $[12,37,44]$. 
Massless case. We now turn to the one-loop partition function associated with the Fronsdal action (2.9). The gauge symmetry forces one to fix a gauge and introduce ghost fields [4], and leads to the following expression for the one-loop term of the partition function:

$$
\log Z=-\frac{1}{2} \log \operatorname{det}\left(-\Delta^{(s)}\right)+\log \operatorname{det}\left(-\Delta^{(s-1)}\right)-\frac{1}{2} \log \operatorname{det}\left(-\Delta^{(s-2)}\right) .
$$

As before, $\Delta^{(s)}$ is the Laplacian on $\mathbb{R}^{D} / \mathbb{Z}$ acting on periodic, traceless, symmetric fields with $s$ indices. The functional determinants can be evaluated exactly as in the massive case, setting $M=0$. In particular, using $\lim _{x \rightarrow 0} x K_{1}(x)=1$, the massless version of the functional determinant $(2.19)$ is

$$
-\log \operatorname{det}\left(-\Delta^{(s)}\right)=\sum_{n \in \mathbb{Z}^{*}} \frac{1}{|n|} \frac{\chi_{s}[n \vec{\theta}, \vec{\epsilon}]}{\prod_{j=1}^{r}\left|1-e^{i n\left(\theta_{j}+i \epsilon_{j}\right)}\right|^{2}} \times \begin{cases}1 & \text { if } D \text { odd } \\ \frac{\Delta z}{\pi|n| \beta} & \text { if } D \text { even }\end{cases}
$$

which has been regularised as discussed in the massive case. The matching (2.21) between $\chi_{s}$ and a character of $\mathrm{SO}(D)$ remains valid, but a sharp difference arises upon including all three functional determinants in (2.25): the combination of $\chi_{s}$ 's is

$$
\chi_{s}[n \vec{\theta}]-2 \chi_{s-1}[n \vec{\theta}]+\chi_{s-2}[n \vec{\theta}] \stackrel{(2.21)-(2.23)}{=} \chi_{\lambda_{s}}[n \vec{\theta}]-\chi_{\lambda_{s-1}}[n \vec{\theta}] .
$$

It is tempting to use (2.23) once more to rewrite this as a character of $\mathrm{SO}(D-2)$, and indeed this is exactly what happens for even $D$ because in this case the rank of $\operatorname{SO}(D-1)$ equals that of $\mathrm{SO}(D-2)$ :

$$
Z[\beta, \vec{\theta}]=\exp \left[\sum_{n=1}^{\infty} \frac{1}{n} \frac{\chi_{\lambda_{s}}^{\mathrm{SO}(D-2)}[n \vec{\theta}, \vec{\epsilon}]}{\prod_{j=1}^{r}\left|1-e^{i n\left(\theta_{j}+i \epsilon_{j}\right)}\right|^{2}} \frac{\Delta z}{\pi n \beta}\right] \quad(\text { even } D) .
$$

If $D$ is odd, however, in going from $\mathrm{SO}(D-1)$ to $\mathrm{SO}(D-2)$, the rank decreases by one unit: expression (2.27) contains one angle too much to be a character of $\mathrm{SO}(D-2)$. As we show in appendix A.3, one can nevertheless write the difference (2.27) as a sum of $\mathrm{SO}(D-2)$ characters with angle-dependent coefficients (see eq. (A.26b)). Namely, let us define

$$
\mathcal{A}_{k}^{r}(\vec{\theta}) \equiv \frac{\left|\cos \left((r-i) \theta_{j}\right)\right|_{\theta_{k}=0}}{\left|\cos \left((r-i) \theta_{j}\right)\right|}, \quad k=1, \ldots, r
$$

where $\left|A_{i j}\right|$ denotes the determinant of an $r \times r$ matrix. Then the rotating one-loop partition function for a massless field with spin $s$ reads

$$
Z[\beta, \vec{\theta}]=e^{-S^{(0)}} \exp \left[\sum_{n=1}^{\infty} \frac{1}{n} \frac{\sum_{k=1}^{r} \mathcal{A}_{k}^{r}(n \vec{\theta}, \vec{\epsilon}) \chi_{\lambda_{s}}^{\mathrm{SO}(D-2)}\left[n \theta_{1}, \ldots, \widehat{n \theta_{k}}, \ldots, n \theta_{r}, \vec{\epsilon}\right]}{\prod_{j=1}^{r}\left|1-e^{i n\left(\theta_{j}+i \epsilon_{j}\right)}\right|^{2}}\right]
$$


where the hat on top of an argument denotes omission. We have also included a spindependent classical action $S^{(0)}$, whose value is a matter of normalisation and is generally taken to vanish, except in $D=3$. In the latter case, $S^{(0)}=-\beta / 8 G$ for $s=2$ (where $G$ is Newton's constant) ensures invariance of the on-shell action under modular transformations of the vacuum [35, 45], in analogy with the similar choice in $\mathrm{AdS}_{3}$ [34]. On the other hand, $S^{(0)}=0$ for all other spins because their vacuum expectation values are assumed to vanish. For $D=3$ the partition function (2.30) can thus be written as

$$
Z[\beta, \vec{\theta}]=e^{\delta_{s, 2} \frac{\beta c_{2}}{24}} \prod_{n=s}^{\infty} \frac{1}{\left|1-e^{i n(\theta+i \epsilon)}\right|^{2}}, \quad c_{2}=3 / G,
$$

and is the flat limit of the analogous higher-spin partition function in $\mathrm{AdS}_{3}$ [4]. We will return to this formula in section 3.2.

The massless partition functions (2.28) and (2.30) are related to the massless limit of (2.24). Indeed, as we show in appendix A.4, it turns out that

$$
\chi_{\lambda_{s}}^{\mathrm{SO}(D-1)}[\vec{\theta}]=\sum_{j=0}^{s} \begin{cases}\sum_{k=1}^{r} \mathcal{A}_{k}^{r}(\vec{\theta}) \chi_{\lambda_{j}}^{\mathrm{SO}(D-2)}\left[\theta_{1}, \ldots, \widehat{\theta_{k}}, \ldots, \theta_{r}\right] & \text { for odd } D \\ \chi_{\lambda_{j}}^{\mathrm{SO}(D-2)}[\vec{\theta}] & \text { for even } D .\end{cases}
$$

Accordingly, the massless limit of a massive partition function with spin $s$ is a product of massless partition functions with spins ranging from 0 to $s$,

$$
\lim _{M \rightarrow 0} Z_{M, s}=\prod_{j=0}^{s} Z_{\text {massless }, j}
$$

consistently with the structure of the action [41]. This result stresses again the role of the functions $\mathcal{A}_{k}^{r}(\vec{\theta})$ defined in (2.29): when the dimension of the space-time is odd one needs angular dependent coefficients because the rank of the little group of massless particles is smaller than the maximum number of angular velocities, so that a single $\operatorname{SO}(D-2)$ character cannot account for all of them.

\section{$2.3 \quad$ Fermionic higher spins}

We now turn to the fermionic analogue of the analysis of the previous subsection. The Euclidean action for a field with spin $s+1 / 2$ (where $s$ is a non-negative integer) and mass $M>0$ can be given either (i) using a symmetric, $\gamma$-traceless spinor field with $s$ spacetime indices and a set of auxiliary fields with no gauge symmetry [46] or (ii) using a set of symmetric spinor fields with $s, s-1, \ldots, 0$ space-time indices and vanishing triple $\gamma$ trace, subject to a gauge symmetry generated by $\gamma$-traceless parameters with $s-1, \ldots, 0$ space-time indices [47]. In the latter case, the action is given again by a sum of actions for massless fields of each of the involved spins, plus a set of cross-coupling terms proportional to the mass. In the limit $M \rightarrow 0$ the quadratic couplings vanish and one is left with a sum of decoupled Fang-Fronsdal actions [20]

$$
S[\psi, \bar{\psi}]=\frac{1}{2} \int d^{D} x \bar{\psi}^{\mu_{1} \ldots \mu_{s}}\left(\mathcal{S}_{\mu_{1} \ldots \mu_{s}}-\frac{1}{2} \gamma_{\left(\mu_{1}\right.} \phi_{\left.\mu_{2} \cdots \mu_{s}\right)}-\frac{1}{2} \delta_{\left(\mu_{1} \mu_{2}\right.} \mathcal{S}_{\left.\mu_{3} \cdots \mu_{s}\right) \lambda}{ }^{\lambda}+\text { h.c. }\right)
$$


where

$$
\mathcal{S}_{\mu_{1} \ldots \mu_{s}}=i\left(\not \partial \psi_{\mu_{1} \ldots \mu_{s}}-\partial_{\left(\mu_{1}\right.} \psi_{\left.\mu_{2} \ldots \mu_{s}\right)}\right),
$$

and one can consider only the field of highest rank/spin.

To compute the partition function for $\psi, \bar{\psi}$ we need to evaluate a path integral (2.1) with the integration measure $\mathcal{D} \psi \mathcal{D} \bar{\psi}$ and $S$ the action (2.34) or its massive analogue. The fermionic fields live on $\mathbb{R}^{D} / \mathbb{Z}$ as defined by the group action (2.7), but in contrast to bosons, they satisfy antiperiodic boundary conditions along the thermal cycle. For a massive field, one thus finds that the partition function is given by

$$
\log Z=\frac{1}{2} \log \operatorname{det}\left(-\Delta^{(s+1 / 2)}+M^{2}\right)-\frac{1}{2} \log \operatorname{det}\left(-\Delta^{(s-1 / 2)}+M^{2}\right),
$$

where $\Delta^{(s+1 / 2)}$ is the Laplacian acting on antiperiodic, symmetric, $\gamma$-traceless spinor fields with $s$ indices on $\mathbb{R}^{D} / \mathbb{Z}$. For massless fields, the gauge symmetry enhancement requires gauge-fixing and ghosts, leading to [6]

$$
\log Z=\frac{1}{2} \log \operatorname{det}\left(-\Delta^{(s+1 / 2)}\right)-\log \operatorname{det}\left(-\Delta^{(s-1 / 2)}\right)+\frac{1}{2} \log \operatorname{det}\left(-\Delta^{(s-3 / 2)}\right) .
$$

To evaluate the necessary functional determinants, we will rely once more on heat kernels and the method of images.

The heat kernel $\mathcal{K}^{A B}{ }_{\mu_{s}, \nu_{s}}$ associated with the operator $\left(-\Delta^{(s+1 / 2)}+M^{2}\right)$ on $\mathbb{R}^{D}$ is the unique solution of the heat equation

$$
\left(\Delta_{(s+1 / 2)}-M^{2}-\partial_{t}\right) \mathcal{K}_{\mu_{s}, \nu_{s}}^{A B}=0, \quad \mathcal{K}^{A B}{ }_{\mu_{s}, \nu_{s}}\left(t=0, x, x^{\prime}\right)=\mathbb{I}_{\mu_{s}, \nu_{s}}^{(F)} \mathbf{1}^{A B} \delta^{(D)}\left(x-x^{\prime}\right) .
$$

Here $\mathcal{K}^{A B}{ }_{\mu_{s}, \nu_{s}}$ is a bispinor in the indices $A$ and $B$, and a symmetric bitensor in the indices $\mu_{s}$ and $\nu_{s}$. (We use again the shorthand $\mu_{s}$ to denote a set of $s$ symmetrised indices.) It is also $\gamma$-traceless in the sense that

$$
\gamma^{\mu} \mathcal{K}_{\mu_{s}, \nu_{s}}=\mathcal{K}_{\mu_{s}, \nu_{s}} \gamma^{\nu}=0
$$

The solution of (2.38) satisfying this requirement is

$$
\mathcal{K}_{\mu_{s}, \nu_{s}}\left(t, x, x^{\prime}\right)=\frac{1}{(4 \pi t)^{D / 2}} e^{-M^{2} t-\frac{1}{4 t}\left|x-x^{\prime}\right|^{2}} \mathbb{I}_{\mu_{s}, \nu_{s}}^{(F)}
$$

where $\mathbb{I}_{\mu_{s}, \nu_{s}}^{(F)}$ is the following bisymmetric, $\gamma$-traceless tensor:

$$
\mathbb{I}_{\mu_{s}, \nu_{s}}^{(F)}=\sum_{k=0}^{\left\lfloor\frac{s}{2}\right\rfloor} \frac{(-1)^{k} 2^{k} k ![D+2(s-k-1)] ! !}{s ![D+2(k-1)] ! !}\left(\delta_{\mu \mu}^{k} \delta_{\mu \nu}^{s-2 k} \delta_{\nu \nu}^{s}-\frac{\delta_{\mu \mu}^{s} \delta_{\mu \nu}^{s-2 k-1} \delta_{\nu \nu}^{s} \gamma_{\mu} \gamma_{\nu}}{D+2(s-k-1)}\right)
$$

Up to the replacement of $\mathbb{I}$ by $\mathbb{I}^{(F)}$, the fermionic heat kernel $(2.40)$ is the same as the bosonic one in eq. (2.14). In particular, $\mathbb{I}^{(F)}$ carries all tensor and spinor indices of the heat kernel. 
To evaluate the determinant of $\left(-\Delta^{(s+1 / 2)}+M^{2}\right)$ on $\mathbb{R}^{D} / \mathbb{Z}$, we use once more the method of images (2.6). As before, we need to keep track of the non-trivial index structure of $\mathcal{K}^{A B}{ }_{\mu_{s}, \nu_{s}}$, which leads to

$$
\mathcal{K}_{\mu_{s}, \alpha_{s}}^{\mathbb{R}^{D} / \mathbb{Z}}\left(t, x, x^{\prime}\right)=\sum_{n \in \mathbb{Z}}(-1)^{n}\left(J^{n}\right)_{\alpha}^{\beta} \ldots\left(J^{n}\right)_{\alpha}{ }^{\beta} U^{n} \mathcal{K}_{\mu_{s}, \beta_{s}}\left(t, x, \gamma^{n}\left(x^{\prime}\right)\right)
$$

where the factor $(-1)^{n}$ comes from antiperiodic boundary conditions, $J$ is the matrix (2.8), and $U$ is a $2^{\lfloor D / 2\rfloor} \times 2^{\lfloor D / 2\rfloor}$ matrix acting on spinor indices and defined by

$$
J^{\alpha}{ }_{\beta}^{\beta}=U \gamma^{\alpha} U^{-1} .
$$

In other words, $U$ is the matrix corresponding to the transformation (2.8) in the spinor representation of $\mathrm{SO}(D)$, and it can be written as

$$
U=\exp \left[\frac{1}{4} \sum_{j=1}^{r} \theta_{j}\left[\gamma_{2 j-1}, \gamma_{2 j}\right]\right]
$$

In particular, a rotation by $2 \pi$ around any given axis maps $\psi$ on $-\psi$, in accordance with the fact that spinors form a projective representation of $\mathrm{SO}(D)$. Note that, using an explicit $D$-dimensional representation of the $\gamma$ matrices, one gets

$$
\operatorname{Tr}\left(U^{n}\right)=2^{\lfloor D / 2\rfloor} \prod_{i=1}^{r} \cos \left(n \theta_{i} / 2\right) .
$$

Now, plugging (2.42) into formula (2.3) for the determinant of $-\Delta^{(s+1 / 2)}$, one obtains a sum of integrals which can be evaluated exactly as in the bosonic case. The only difference with respect to bosons comes from the spin structure, and the end result is

$$
-\log \operatorname{det}\left(-\Delta^{(s+1 / 2)}+M^{2}\right)=\sum_{n \in \mathbb{Z}^{*}} \frac{(-1)^{n}}{|n|} \frac{\chi_{s}^{(F)}[n \vec{\theta}, \vec{\epsilon}]}{\prod_{j=1}^{r}\left|1-e^{i n\left(\theta_{j}+i \epsilon_{j}\right)}\right|^{2}} \times \begin{cases}e^{-|n| \beta M} & D \text { odd }, \\ \frac{M \Delta z}{\pi} K_{1}(|n| \beta M) & D \text { even },\end{cases}
$$

where we have discarded a volume divergence independent of all chemical potentials (as on page 8 ), and where

$$
\chi_{s}^{(F)}[n \vec{\theta}, \vec{\epsilon}]=\left(J^{\mu \alpha}\right)^{s} \operatorname{Tr}\left[\mathbb{I}_{\mu_{s}, \alpha_{s}}^{(F)}\right]
$$

is the fermionic analogue of (2.20), with the same regularisation as above. This result takes exactly the same form as (2.19), up to the replacement of $\chi_{s}$ by $\chi_{s}^{(F)}$. In appendices B.1 and B.2, we show that

$$
\chi_{s}^{(F)}[n \vec{\theta}] \stackrel{\text { B.1\&B. } 2}{=} \begin{cases}\chi_{\lambda_{s}^{(F)}}[n \vec{\theta}] & \text { for odd } D, \\ \chi_{\lambda_{s}^{(F)}}[n \vec{\theta}, 0] & \text { for even } D,\end{cases}
$$

where the term on the right-hand side is the character of an irreducible representation of $\mathrm{SO}(D)$ with highest weight $\lambda_{s}^{(F)}=(s+1 / 2,1 / 2, \ldots, 1 / 2)$ (written here in the dual basis of the Cartan subalgebra of $\mathfrak{s o}(D)$ described above $(2.21))$. 
Having computed the required functional determinants on $\mathbb{R}^{D} / \mathbb{Z}$, we can now write down the partition functions given by (2.36) and (2.37). In the massive case, the difference of Laplacians acting on fields with spins $(s+1 / 2)$ and $(s-1 / 2)$ produces the difference of two factors (2.48), with labels $s$ and $s-1$. It turns out that identity (2.23) still holds if we replace $\lambda_{s}$ and $\lambda_{s-1}$ by their fermionic counterparts, $\lambda_{s}^{(F)}$ and $\lambda_{s-1}^{(F)}$. (The proof of this statement follows the exact same steps as in the bosonic case described in appendix A.3, up to obvious replacements that account for the change in the highest weight vector.) Accordingly, the rotating one-loop partition function of a massive field with spin $s+1 / 2$ is

$$
Z[\beta, \vec{\theta}]=\exp \left[\sum_{n=1}^{\infty} \frac{(-1)^{n}}{n} \frac{\chi_{\lambda_{s}^{(F)}}^{\mathrm{SO}(D-1)}[n \vec{\theta} \vec{\epsilon}]}{\prod_{j=1}^{r}\left|1-e^{i n\left(\theta_{j}+i \epsilon_{j}\right)}\right|^{2}} \times\left\{\begin{array}{ll}
e^{-n \beta M} & (D \text { odd }) \\
\frac{M \Delta z}{\pi} K_{1}(n \beta M) & (D \text { even })
\end{array}\right] .\right.
$$

In the massless case we must take into account one more difference of characters, namely (2.27) with $\lambda_{s}$ replaced by $\lambda_{s}^{(F)}$. Again, this difference can be written as a combination of $\mathrm{SO}(D-2)$ characters (the proof being almost the same as in appendix A.3), and the partition function of a massless field with spin $s+1 / 2$ exactly takes the form (2.28) or (2.30) (for $D$ even or odd, respectively) with an additional factor of $(-1)^{n}$ in the sum over $n$, and the replacement of $\lambda_{s}$ by $\lambda_{s}^{(F)}$. In particular, for $D=3$, the massless partition function can be written as

$$
Z=\prod_{n=s}^{\infty}\left|1+e^{i(n+1 / 2)(\theta+i \epsilon)}\right|^{2},
$$

an expression that we will use in section 3.3 and that can be recovered as the flat limit of the AdS result [6]. One can also verify that relation (2.33) remains true for fermionic partition functions.

\subsection{Relation to Poincaré characters}

In this subsection we show that all one-loop partition functions displayed above can be written as exponentials of (sums of) Poincaré characters. Along the way we briefly review the construction of induced representations of semi-direct products that will be useful also for section 3. We refer e.g. to $[48,49]$ for a more self-contained presentation.

Representations of semi-direct products. Let $G$ be a group, $A$ an Abelian vector group, $\sigma$ a representation of $G$ in $A$. Then the semi-direct product of $G$ and $A$ (with respect to $\sigma$ ) is the group denoted $G \ltimes_{\sigma} A$ (or simply $G \ltimes A$ ) whose elements are pairs $(f, \alpha) \in G \times A$ and whose group operation is $(f, \alpha) \cdot(g, \beta)=\left(f \cdot g, \alpha+\sigma_{f} \beta\right)$. The Poincaré groups are precisely of that type, with $G$ a Lorentz group or a spin group, and $A$ a group of translations, the action $\sigma$ then being the vector representation of the Lorentz group.

It turns out that all irreducible, unitary representations of a semi-direct product are induced representations analogous to those of the Poincaré group [50-52]. They are classified by orbits of "momenta" belonging to the dual space of the Abelian group. Concretely, take some momentum vector $p \in A^{*}$ and denote by $\mathcal{O}_{p}=\{f \cdot p \mid f \in G\}$ its orbit under 
$G$. Let also $\mathcal{R}$ be some unitary representation of the corresponding little group $G_{p}$, and denote its character by $\chi_{\mathcal{R}}$. Then, according to the Frobenius formula [53], the character of the associated induced representation is

$$
\chi[(f, \alpha)]=\int_{\mathcal{O}_{p}} d \mu(q) e^{i\langle q, \alpha\rangle} \chi_{\mathcal{R}}\left[g_{q}^{-1} f g_{q}\right] \delta(q, f \cdot q),
$$

where $\mu$ is some (quasi-invariant) measure on $\mathcal{O}_{p}, \delta$ is the associated Dirac distribution, and the $g_{q}$ 's are boosts such that $g_{q} \cdot p=q$. One can verify that the value of $\chi[(f, \alpha)]$ is independent of the choice of $\mu$, and that $\chi[(f, \alpha)]$ vanishes if $f$ is not conjugate to an element of the little group (see e.g. [49]). We now apply this formula to the Poincaré group, while in the next section we will use it to evaluate characters of flat $\mathcal{W}_{N}$ algebras and of supersymmetric extensions of $\mathfrak{b m s}_{3}$.

Poincaré groups and induced representations. The connected Poincaré group in $D$ dimensions is a semi-direct product $\operatorname{SO}(D-1,1)^{\uparrow} \ltimes \mathbb{R}^{D}$, where $\operatorname{SO}(D-1,1)^{\uparrow}$ is the proper, orthochronous Lorentz group and $\mathbb{R}^{D}$ is the group of space-time translations. The classification of (projective) irreducible, unitary representations of this group follows from the classification of orbits of momenta in terms of the value of the mass squared [50]. In particular, massive orbits have little group $\mathrm{SO}(D-1)$, while massless orbits have little group $\mathrm{SO}(D-$ $2) \ltimes \mathbb{R}^{D-2}$. We now evaluate the characters of irreducible representations of the Poincaré group. To our knowledge, Poincaré characters have been discussed previously in [49, 54-56].

Massive Poincaré characters. Consider a massive momentum orbit $\mathcal{O}_{p}$ with positive energy, where $p$ is the momentum of a massive particle at rest, say $p=(M, 0, \ldots, 0) \in \mathbb{R}^{D}$. Let $\mathcal{R}$ be an irreducible, unitary representation of the corresponding little group $\mathrm{SO}(D-1)$ labelled by a highest weight $\lambda$ and pick a measure $\mu$ on $\mathcal{O}_{p}$. Since the character (2.51) vanishes whenever $f$ is not conjugate to an element of the little group, we will take $f$ to be the rotation (2.8). Note that we could have chosen any other rotation by the same angles without affecting the result, since the value of the character depends only on the conjugacy class of the group element at which it is evaluated.

When $D$ is odd, and provided all angles $\theta_{1}, \ldots, \theta_{r}$ are non-zero, the delta function $\delta(q, f \cdot q)$ in $(2.51)$ localises the integral over the orbit to a single point - namely the momentum in the rest frame, $p$. The character (2.51) then reduces to

$$
\chi[(f, \alpha)]=e^{i M \alpha^{0}} \chi_{\mathcal{R}}[f] \int_{\mathcal{O}_{p}} d \mu(q) \delta(q, f \cdot q),
$$

where $\chi_{\mathcal{R}}[f]=\chi_{\lambda}^{\mathrm{SO}(D-1)}[\vec{\theta}]$ because the rotation (2.8) belongs to the little group. To integrate the delta function, we use the spatial components of the momentum $q$ as coordinates on the orbit; in terms of these coordinates the integral reads

$$
\int_{\mathcal{O}_{p}} d \mu(q) \delta(q, f \cdot q)=\int_{\mathbb{R}^{D-1}} d q_{1} \ldots d q_{D-1} \delta^{(D-1)}(\mathbf{q}-f \cdot \mathbf{q})=\prod_{j=1}^{r} \frac{1}{\left|1-e^{i \theta_{j}}\right|^{2}}
$$


where we have chosen the flat Lebesgue measure on $\mathcal{O}_{p}$ owing to the $\mu$-independence of the result. The character (2.52) then becomes

$$
\chi[(f, \alpha)]=e^{i M \alpha^{0}} \chi_{\lambda}^{\mathrm{SO}(D-1)}[\vec{\theta}] \prod_{j=1}^{r} \frac{1}{\left|1-e^{i \theta_{j}}\right|^{2}} .
$$

In order to represent a particle with spin $s$, we choose the weight $\lambda$ to be $\lambda_{s}=(s, 0, \ldots, 0)$ (in the dual basis of the Cartan subalgebra of $\mathfrak{s o}(D-1)$ described above $(2.21)$ ). With this choice, expression (2.54) actually appears in the exponent of (2.24): taking $\alpha^{0}=i \beta$, we can rewrite the rotating one-loop partition function for a massive field with spin $s$ (in odd $D$ ) as the exponential of a sum of Poincaré characters:

$$
Z_{M, s}[\beta, \vec{\theta}]=\exp \left[\sum_{n=1}^{\infty} \frac{1}{n} \chi_{M, s}[n \vec{\theta}, i n \beta]\right] .
$$

The series in the exponent is divergent for real $\theta_{i}$ 's. This divergence can be regularised by adding suitable imaginary parts to these angles, as explained below (2.19).

In $D=3$ space-time dimensions, the massive little group is $\mathrm{SO}(2) \cong \mathrm{U}(1)$ and its character for an irreducible representation with spin $s$ is $e^{i s \theta}$, so that (2.54) boils down to

$$
\chi_{M, s}\left[\left(\operatorname{rot}_{\theta}, \alpha=i \beta\right)\right]=e^{-\beta M+i \theta s} \frac{1}{\left|1-e^{i \theta}\right|^{2}},
$$

where we take $\alpha$ to be a Euclidean time translation by $\beta .^{4}$ In the next section we will show that the characters of flat $\mathcal{W}_{N}$ algebras (and of supersymmetric versions of $\mathfrak{b m s}_{3}$ ) are natural extensions of this formula.

When $D$ is even, the situation is more involved because the integral localises to a line instead of a point. For $\alpha$ being an Euclidean time translation by $\beta$, this leads to a non-trivial, infrared-divergent integral

$$
\int_{-\infty}^{+\infty} d k \delta(k-k) e^{-\beta \sqrt{M^{2}+k^{2}}}=\frac{M \Delta z}{\pi} K_{1}(\beta M)
$$

where we interpret $\delta(0)$ as $\Delta z / 2 \pi$, with $\Delta z$ the height of the rotating box along the space direction dual to $k$, and $K_{1}$ is a modified Bessel function of the second kind. Accordingly, for even $D$, the character of a rotation (2.8) accompanied by a Euclidean time translation by $\beta$ in a massive representation of the Poincaré group is

$$
\chi[(f, \alpha=i \beta)]=\frac{M \Delta z}{\pi} K_{1}(\beta M) \chi_{\lambda}^{\mathrm{SO}(D-1)}[\vec{\theta}] \prod_{j=1}^{r} \frac{1}{\mid 1-e^{\left.i \theta_{j}\right|^{2}}} .
$$

For $\lambda=\lambda_{s}=(s, 0, \ldots, 0)$, this expression coincides with the combinations appearing in the partition function (2.24) upon writing the latter as (2.55). The same matching works for massive fermionic fields in all space-time dimensions when replacing $\lambda_{s}$ by $\lambda_{s}^{(F)}=$ $(s+1 / 2,1 / 2, \ldots, 1 / 2)$.

\footnotetext{
${ }^{4} \mathrm{~A}$ parity-invariant version of this expression is obtained upon replacing $e^{i s \theta}$ by $2 \cos (s \theta)$.
} 
Massless Poincaré characters (discrete spin). The little group for particles with vanishing mass is $\mathrm{SO}(D-2) \ltimes \mathbb{R}^{D-2}$. A massless particle is said to have discrete spin if the space of its spin degrees of freedom forms a finite-dimensional representation of the little group, in which all boosts spanning $\mathbb{R}^{D-2}$ are represented trivially. In this subsection we will focus on such particles, relegating some comments on continuous spin particles to section 4. Once more, we will treat separately even and odd space-time dimensions.

In even space-time dimensions, any rotation in $\mathrm{SO}(D-1)$ is conjugate to an element of $\mathrm{SO}(D-2)$ (in accordance with the fact that these groups have the same rank). In fact, for even $D$, the rotation (2.8) belongs to the subgroup $\mathrm{SO}(D-2)$ of the Lorentz group leaving invariant the momentum of a massless particle moving along the $z$ axis, so the massless characters of Poincaré in even $D$ are just the limit $M \rightarrow 0$ of $(2.57)$, with the character of $\mathrm{SO}(D-1)$ replaced by a character of $\mathrm{SO}(D-2)$. Using also $\lim _{x \rightarrow 0} x K_{1}(x)=1$, we get

$$
\chi[\vec{\theta}, \beta]=\frac{\Delta z}{\pi \beta} \chi_{\lambda}^{\mathrm{SO}(D-2)}[\vec{\theta}] \prod_{j=1}^{r} \frac{1}{\left|1-e^{i \theta_{j}}\right|^{2}},
$$

which is indeed the expression appearing in the partition function (2.28) upon writing it as (2.55).

In odd space-time dimensions, $\mathrm{SO}(D-2)$ has lower rank than $\mathrm{SO}(D-1)$, so the rotation (2.8) is not, in general, conjugate to an element of the massless little group: it has one angle too much, and whenever all angles $\theta_{1}, \ldots, \theta_{r}$ are non-zero, the character (2.51) vanishes. The only non-trivial irreducible character arises when at least one of the angles $\theta_{1}, \ldots, \theta_{r}$ vanishes, say $\theta_{r}=0$. Then the arguments explained above can be applied once more, the only subtlety being that the two spatial components $\left(k_{1}, k_{2}\right)$ of momentum that are not rotated produce an integral

$$
\int_{\mathbb{R}^{2} \backslash\{0\}} d k_{1} d k_{2} e^{-\beta \sqrt{k_{1}^{2}+k_{2}^{2}}} \delta\left(k_{1}-k_{1}\right) \delta\left(k_{2}-k_{2}\right)=\frac{\Delta z \Delta z^{\prime}}{2 \pi \beta^{2}},
$$

where we have once more interpreted the delta functions evaluated at zero as infrareddivergent factors. The character of an irreducible massless representation of the Poincaré group in odd space-time dimension $D$ is thus

$$
\chi\left[\theta_{1}, \ldots, \theta_{r-1}, \beta\right]=\chi_{\lambda}^{\mathrm{SO}(D-2)}\left[\theta_{1}, \ldots, \theta_{r-1}\right] \frac{\Delta z \Delta z^{\prime}}{2 \pi \beta^{2}} \prod_{j=1}^{r-1} \frac{1}{\mid 1-e^{\left.i \theta_{j}\right|^{2}}} .
$$

However, comparison with (2.30) reveals a mismatch: the partition function does not take the form (2.55) in terms of the massless characters (2.61); in field theory, all $r$ angles $\theta_{i}$ may be switched on simultaneously! To accommodate for this one can resort to the angle-dependent coefficients $\mathcal{A}_{k}^{r}(\vec{\theta})$ introduced in (2.29). Also in this context, one can understand the origin of these coefficients through the massless limit of the character (2.54). Using relation (2.32), the product of massless partition functions with spins ranging from zero to $s$ can be written as (2.55), where the characters on the right-hand side are massless limits of massive Poincaré characters. However, it is not clear to us how the quantities appearing in the exponent of (2.30) can be related directly to Poincaré characters without invoking a massless limit. 
Remark. The relation (2.55) between one-loop partition functions and characters of the underlying space-time isometry group is not new. From a physical standpoint, it is merely the statement that a free field is a collection of harmonic oscillators, one for each value of momentum: the index $n$ then labels the oscillator modes, while the integral over momenta is the one in the Frobenius character formula (2.51). In particular, standard, nonrotating one-loop partition functions are exponentials of sums of characters of (Euclidean) time translations. This relation has also been observed in AdS [37, 44, 57]; our partition functions are flat limits of these earlier results, up to the even-dimensional regularisation subtlety mentioned below eq. (2.20). Note that this issue already emerges at the level of characters: although most of $(2.58)$ is a flat limit of an $\mathrm{SO}(D-1,2)$ character, it is not clear how to regularise the divergences that pop up when one of the angles vanishes in order to recover our regulators $\Delta z$. This problem would also appear in odd $D$ if one or more angles were set to zero.

\section{$3 \quad$ Three-dimensional applications}

In this section we exhibit the matching between certain combinations of higher-spin partition functions in three dimensions and vacuum characters of suitable asymptotic symmetry algebras. We start by reviewing the purely gravitational setting studied in [35, 48, 49, 58], before moving on to illustrative classes of bosonic (section 3.2) and supersymmetric (section 3.3) higher-spin theories [30-32]. In the latter cases the characterisation of representations of the asymptotic symmetry algebras is subtler; nevertheless, motivated by the analogy with the gravitational setup, we propose to extend to this context several tools of the theory of induced representations recalled in section 2.4 .

\section{1 $\mathrm{BMS}_{3}$ particles and induced $\mathfrak{b m s}_{3}$ modules}

The asymptotic symmetries of three-dimensional gravity without cosmological constant at null infinity are given by the $\mathfrak{b m s}_{3}$ algebra [27-29], whose representations are most conveniently analysed from the viewpoint of the underlying $\mathrm{BMS}_{3}$ group. The unitary representations of this group — or $\mathrm{BMS}_{3}$ particles — have been studied in detail in [48, 58]. Here we briefly recall some results of this analysis that we are going to extend to the higher spin setup. We also present a characterisation of induced representations at the Lie-algebraic level, that allows us to make contact with earlier proposals on the structure of unitary representations of flat $\mathcal{W}$ algebras [38].

$\mathrm{BMS}_{3}$ representations. The $\mathrm{BMS}_{3}$ group is a semi-direct product $G \ltimes \mathfrak{g}$, where $G$ is the Virasoro group (consisting of superrotations) and $\mathfrak{g}$ is its Lie algebra, seen as an Abelian vector group ${ }^{5}$ (consisting of supertranslations):

$$
\mathrm{BMS}_{3}=\operatorname{Diff}\left(S^{1}\right) \ltimes \operatorname{Vect}\left(S^{1}\right) .
$$

\footnotetext{
${ }^{5}$ Below we will use the more accurate notation $G \ltimes_{\mathrm{Ad}} \mathfrak{g}_{\mathrm{Ab}}$, where $\mathfrak{g}_{\mathrm{Ab}}$ is an Abelian vector group isomorphic to $\mathfrak{g}$ as a vector space, and the subscript "Ad" indicates that $G$ acts on $\mathfrak{g}_{\mathrm{Ab}}$ according to the adjoint action.
} 
Accordingly, the duals of (centrally extended) supertranslations are pairs $\left(p(\varphi), c_{2}\right)$, where $p(\varphi)$ is a supermomentum (it is a function on the circle) and $c_{2}$ is a central charge taking the value $c_{2}=3 / G$ in Einstein gravity [28] (see the $\mathfrak{b m s}_{3}$ Lie algebra (3.4)). Each induced representation of $\mathrm{BMS}_{3}$ is associated with the orbit of such a pair $\left(p(\varphi), c_{2}\right)$ under the action of $\operatorname{Diff}\left(S^{1}\right)$, i.e. with a coadjoint orbit of the Virasoro group [59,60]. The states of a $\mathrm{BMS}_{3}$ particle are wavefunctions in supermomentum space, and given any (quasiinvariant) measure on the orbit, the corresponding representation is unitary (see e.g. [49]). The associated character is given by the Frobenius formula (2.51), with the subtlety that the supermomentum integral is taken over an infinite-dimensional manifold. However, as recalled in section 2.4, the character vanishes whenever the element of the group which is used to determine the character in the pair $(f, \alpha)$ is not conjugate to an element of the little group. When $f$ is (conjugate to) a rotation by some non-zero angle $\theta$, the integral in (2.51) localises, so that one can compute the character explicitly [49]. For a massive $\mathrm{BMS}_{3}$ particle with mass $M$ and spin $s$, i.e. for a representation whose orbit contains a constant supermomentum $p_{0}=M-c_{2} / 24$ with $M>0$, the character is given by

$$
\chi_{M, s}\left[\left(f=\operatorname{rot}_{\theta}, \alpha=i \beta\right)\right]=e^{-\beta M+i \theta s} \frac{1}{|1-q|^{2}} \cdot e^{\beta c_{2} / 24} \frac{1}{\prod_{n=2}^{\infty}\left|1-q^{n}\right|^{2}},
$$

where $q=e^{i(\theta+i \epsilon)}$ with a factor $i \epsilon$ added to ensure convergence of the infinite product. This expression is the product of the massive Poincare character (2.56) with the vacuum $\mathrm{BMS}_{3}$ character

$$
\chi_{\operatorname{vac}}^{\mathrm{BMS}}\left[\left(\operatorname{rot}_{\theta}, i \beta\right)\right]=e^{\beta c_{2} / 24} \frac{1}{\prod_{n=2}^{\infty}\left|1-e^{i n(\theta+i \epsilon)}\right|^{2}},
$$

which coincides with the one-loop partition function of gravitons given by eq. (2.31) for $s=2[35]$.

The main lessons to be drawn from the previous considerations are that (i) representations of the $\mathrm{BMS}_{3}$ group are labelled by orbits of supermomenta and (ii) even if the classification of such orbits requires a detailed knowledge of the finite (as opposed to infinitesimal) transformation laws of supermomenta under superrotations, these details are not relevant for evaluating the characters of the corresponding representations in all cases in which the integral localises. We focused here on representations of the $\mathrm{BMS}_{3}$ group, but by differentiating them at the identity one can gain insights on the corresponding representations of the $\mathfrak{b m s}_{3}$ algebra. By suitably generalising the notion of coadjoint orbit to the higher-spin context, in section 3.2 we will indeed propose a (partial) classification of unitary representations of certain flat $\mathcal{W}$ algebras and compute the associated characters, which are to be matched with the one-loop partition functions computed in section 2 . We are now going to investigate the structure of $\mathfrak{b m s}_{3}$ representations in order to simplify the comparison between our group-inspired classification and previous proposals [38].

Induced $\mathfrak{b m s}_{3}$ modules. The $\mathfrak{b m s}_{3}$ algebra is generated by two infinite families of superrotation and supertranslation generators $J_{m}$ and $P_{m}$ (where $m$ is an integer) corresponding to Fourier modes of vector fields on the circle, together with two central charges $Z_{1}$ and $Z_{2}$. In any irreducible representation of the algebra, these charges are proportional to the 
identity operator, so from now on we will replace $Z_{1}$ and $Z_{2}$ by numbers $c_{1}, c_{2}$. In terms of these quantities, the commutation relations of the $\mathfrak{b m s}_{3}$ algebra read $[28,29,61]$

$$
\begin{aligned}
& {\left[J_{m}, J_{n}\right]=(m-n) J_{m+n}+\frac{c_{1}}{12} m\left(m^{2}-1\right) \delta_{m+n, 0},} \\
& {\left[J_{m}, P_{n}\right]=(m-n) P_{m+n}+\frac{c_{2}}{12} m\left(m^{2}-1\right) \delta_{m+n, 0},} \\
& {\left[P_{m}, P_{n}\right]=0 .}
\end{aligned}
$$

Note that this algebra is a semi-direct sum

$$
\mathfrak{b m s}_{3}=\mathfrak{v i r} \oplus_{\mathrm{Ad}}(\mathfrak{v i r})_{\mathrm{Ab}},
$$

where $\mathfrak{v i r}$ is the Virasoro algebra and $(\mathfrak{v i r})_{\mathrm{Ab}}$ denotes an Abelian Lie algebra isomorphic, as a vector space, to $\mathfrak{v i r}$; the action of the Virasoro algebra on its Abelian counterpart is the adjoint action, as indicated by the subscript "Ad". One way to obtain the algebra (3.4) is to take an İnönü-Wigner contraction [62] of two commuting copies of the Virasoro algebra, which can be physically interpreted as a flat/ultrarelativistic limit (see eq. (3.11)). One can indeed define

$$
P_{n} \equiv \frac{1}{\ell}\left(L_{n}+\bar{L}_{-n}\right), \quad J_{n} \equiv L_{n}-\bar{L}_{-n}, \quad c_{1} \equiv c-\bar{c}, \quad c_{2} \equiv \frac{c+\bar{c}}{\ell},
$$

where $L_{n}$ and $\bar{L}_{n}$ denote the generators of the two Virasoro algebras with central charges $c$ and $\bar{c}$ and $\ell$ is a length scale. In the limit $\ell \rightarrow \infty$ one recovers (3.4).

The unitary representations of the group corresponding to the algebra (3.4) are induced, in the sense explained above. Here we wish to understand the differential of these representations at the identity, that is, the associated representations of $\mathfrak{b m s}_{3}$. For definiteness, let us consider a massive $B M S_{3}$ particle, whose orbit contains a constant supermomentum $p_{0}=M-c_{2} / 24$ with $M>0$. A convenient basis of the particle's Hilbert space consists of plane waves, that is, wavefunctions with definite supermomentum (see e.g. section 2.3 of [48]). In particular, there is a wavefunction whose supermomentum is the constant $p_{0}$, representing the state of the particle at rest. We will call this particular wavefunction the rest-frame state of the representation and denote it by $|M, s\rangle$, where $s \in \mathbb{R}$ is the spin of the particle, i.e. the eigenvalue of the $J_{0}$ generator. By construction, it transforms as follows under a finite supertranslation $\alpha$ :

$$
U(\alpha)|M, s\rangle=e^{i M \alpha^{0}}|M, s\rangle, \quad U(\alpha)=\exp \left[i \sum_{n \in \mathbb{Z}} P_{n} \alpha^{n}\right] .
$$

Here $\alpha(\varphi)=\sum_{n \in \mathbb{Z}} e^{i n \varphi} \alpha^{n}$ is a real function on the circle and $U(\alpha)$ is a unitary operator, so that $P_{n}^{\dagger}=P_{-n}$. By differentiating with respect to $\alpha$, one obtains

$$
P_{0}|M, s\rangle=M|M, s\rangle, \quad P_{n}|M, s\rangle=0 \text { for } n \neq 0, \quad J_{0}|M, s\rangle=s|M, s\rangle,
$$

where the last condition comes from the definition of $|M, s\rangle$. The remaining superrotation generators $J_{m}$ (with $m \neq 0$ ), when acting on the rest-frame state, produce new states of the form

$$
J_{n_{1}} \cdots J_{n_{k}}|M, s\rangle
$$


where $n_{1}, \ldots, n_{k}$ are arbitrary non-zero integers such that $n_{1} \geq \ldots \geq n_{k}$, and $k=0,1,2, \ldots$ These additional states arise because finite superrotations act on wavefunctions as unitary operators

$$
U(\omega)=\exp \left[i \sum_{n \in \mathbb{Z}} J_{n} \omega^{n}\right]
$$

where the complex coefficients $\omega^{n}=\left(\omega^{-n}\right)^{*}$ are generalizations of the rapidity parameter of special relativity, and $J_{n}^{\dagger}=J_{-n}$. In particular, in contrast to Virasoro representations, the rest-frame state does not satisfy any highest-weight condition, reflecting the fact that it can be boosted in any direction.

We will call states of the form (3.9) boosted states. We also call induced module (with mass $M$ and spin $s$ ) the space $\mathcal{H}_{\mathfrak{b m s}}$ whose basis consists of the rest-frame state $|M, s\rangle$ and its boosted counterparts (3.9); it forms an irreducible representation of the $\mathfrak{b m s}_{3}$ algebra (3.4), and it is unitary by construction, since it arises from a unitary representation of the $\mathrm{BMS}_{3}$ group. Similarly, the rest-frame state $|0\rangle$ of the vacuum induced module satisfies (3.8) with $M=s=0$ together with the additional condition $J_{ \pm 1}|0\rangle=0$, ensuring Lorentz-invariance. Boosted vacua are again of the form (3.9), but with all $n_{i}$ 's different from $-1,0$ and 1 .

Since the algebra (3.4) emerges from the İnönü-Wigner contraction of the conformal algebra via the redefinitions (3.6) and the limit $\ell \rightarrow \infty$, one can also motivate the representations above by a limiting procedure. For instance, in [49] it has been shown that one can recover the character (3.2) as a flat limit of characters of non-degenerate highest-weight representations of the Virasoro algebras generated by the $L_{n}$ and $\bar{L}_{n}$ of (3.6). To this end, one has to write the modular parameter as $\tau=\frac{1}{2 \pi}(\theta+i \beta / \ell)$ and let the highest weights $h$ and $\bar{h}$ depend on $\ell$ in such a way that the parameters

$$
M=\lim _{\ell \rightarrow \infty} \frac{1}{\ell}(h+\bar{h}), \quad s=\lim _{\ell \rightarrow \infty}(h-\bar{h})-\frac{c_{1}}{24}
$$

be finite. The conditions (3.8) on the rest-frame state $|M, s\rangle$ can be seen to emerge from this limit as well, since the Virasoro highest-weight conditions translate into

$$
\begin{array}{cc}
\frac{1}{\ell} L_{n}|h, \bar{h}\rangle=\frac{1}{2}\left(P_{n}+\frac{1}{\ell} J_{n}\right)|h, \bar{h}\rangle=0 \stackrel{\ell \rightarrow \infty}{\longrightarrow} P_{n}|M, s\rangle=0 \quad \text { for } n>0, \\
\frac{1}{\ell} \bar{L}_{n}|h, \bar{h}\rangle=\frac{1}{2}\left(P_{-n}-\frac{1}{\ell} J_{-n}\right)|h, \bar{h}\rangle=0 \stackrel{\ell \rightarrow \infty}{\longrightarrow} P_{-n}|M, s\rangle=0 \quad \text { for } n>0,
\end{array}
$$

i.e. in the second condition in (3.8), while no constraints are imposed on the $J_{n}$. Thus, in the limit one does not keep the full Virasoro highest-weight conditions, but only their leading term in a large $\ell$ expansion. This is analogous to what one does at the level of the algebra: after the redefinition (3.6) the commutator of two $P_{n}$ 's would be proportional to $\ell^{-2}$, but in the limit $\ell \rightarrow \infty$ one omits the right-hand side to get (3.4c). We stress, however, that the definition of the rest-frame state holds independently of the limit and follows from the theory of induced representations applied to the $\mathrm{BMS}_{3}$ group. In this sense the difference between highest-weight and rest-frame conditions reflects the very different structure of $\mathfrak{b m s}_{3}$ and Virasoro representations, generalising the difference between Poincaré and $\mathfrak{s o}(2,2)$ representations. For a detailed analysis see [63]. 
To complete the characterisation of the induced module one would like to recover the character (3.2) by computing the trace of $e^{-\beta H+i \theta J}$ over the space defined by (3.8) and (3.9). In spite of the link between (3.2) and the flat limit of Virasoro characters recalled above, it is however not clear to us how to define a trace over induced modules that produces the desired result. This is not completely surprising since the character formula (3.2) entails the ad hoc regularisation obtained by adding a small imaginary part to each angle. A natural counterpart of the divergence of the infinite product in (3.2) is the infinite multiplicity of each eigenvalue of $P_{0}$ and $J_{0}$ in the induced module, that should be regularised in some way. One should keep in mind, however, that induced modules do not capture all the features of induced representations. This is mainly due to the fact that the energy spectrum of a $\mathrm{BMS}_{3}$ particle is continuous, so that, for instance, one cannot expect to be able to compute the characters of a pure supertranslation solely from the infinitesimal picture. To avoid pathologies one should stick to the characterisation of the Hilbert space of each representation in terms of wavefunctions on orbits of supermomenta rather than in terms of induced modules.

In spite of its limitations, the previous infinitesimal picture is useful to understand how the representations of the $\mathfrak{b m s}_{3}$ algebra discussed in $[38,64]$ fit within the classification which emerges from that of induced representations of the $\mathrm{BMS}_{3}$ group. As discussed above, unitary $\mathrm{BMS}_{3}$ representations are labelled by orbits of pairs $\left(p(\varphi), c_{2}\right)$, where $c_{2}$ is non-zero in Einstein gravity. Nevertheless, one may consider the induced representation associated with the trivial orbit of $p(\varphi)=c_{2}=0$, whose little group is the whole Virasoro group generated by superrotations. In that representation, all supermomenta are set to zero, and the only non-trivial piece comes from the representation of the little group, which is just a standard Virasoro highest-weight representation obtained by starting from a highest-weight state $|s\rangle$ such that

$$
J_{0}|s\rangle=s|s\rangle, \quad J_{m}|s\rangle=0 \text { for } m>0 .
$$

In the Poincaré group, the analogue of this construction would consist in building a unitary representation where all translations act trivially, while Lorentz transformations are represented in a non-trivial, unitary way. It turns out that all unitary representations of $\mathfrak{b m s}_{3}$ (and its higher-spin extensions) considered in [38,64] were of this type. The authors attempted to build representations by enforcing the conditions (3.14) while replacing $|s\rangle$ by a state $|M, s\rangle$, with $M$ the energy of the state. Upon switching on the central charge $c_{2}$, they concluded that unitary representations arise only if $M=c_{2}=0$. But as we can see from our earlier considerations, this had to be so: the highest-weight conditions (3.14) rely on the assumption that superrotations are represented as in a usual CFT, which occurs only for $M=c_{2}=0$. By contrast, for non-zero $c_{2}$, the suitable conditions are not (3.14), but the rest-frame conditions (3.8).

\subsection{Characters of flat $\mathcal{W}_{N}$ algebras}

We now move to the asymptotic symmetry algebras that arise at null infinity for higher-spin theories in flat space. We propose a way to characterise their unitary representations and compute the associated characters, showing in particular that vacuum characters match 
certain combinations of the one-loop partition functions displayed in section 2.2. The coadjoint representation of standard $\mathcal{W}_{N}$ algebras [65-67] plays a key role in our analysis, so we start by first reviewing results from the AdS context.

Higher spins in $\mathbf{A d S}_{\mathbf{3}}$ and the $\mathcal{W}_{\mathbf{3}}$ algebra. Asymptotic symmetries of higher-spin theories in three dimensions were first studied in $\mathrm{AdS}_{3}$ [23-26], where they typically span the direct sum of two non-linear $\mathcal{W}$ algebras. Here we focus on models including fields with spin ranging from 2 to $N .{ }^{6}$ When $N=3$, the asymptotic symmetries are generated by gauge transformations specified by four arbitrary, $2 \pi$-periodic functions $\left(X\left(x^{+}\right), \xi\left(x^{+}\right)\right)$and $\left(\bar{X}\left(x^{-}\right), \bar{\xi}\left(x^{-}\right)\right)$of the light-cone coordinates $x^{ \pm}$on the boundary of $\mathrm{AdS}_{3}$. In particular, the functions $X\left(x^{+}\right)$and $\bar{X}\left(x^{-}\right)$generate conformal transformations [21, 22]. Since the results are left-right symmetric, we focus on the left-moving sector. The surface charge associated with a transformation $(X, \xi)$ then takes the form [24]

$$
Q_{(X, \xi)}[p, \rho]=\frac{1}{2 \pi} \int_{0}^{2 \pi} d \varphi[X(\varphi) p(\varphi)+\xi(\varphi) \rho(\varphi)]
$$

where $\varphi=\left(x^{+}-x^{-}\right) / 2$, while $p(\varphi)$ and $\rho(\varphi)$ are two arbitrary, $2 \pi$-periodic functions specifying a solution of the field equations at fixed time. In fact, if we think of the pair $(X, \xi)$ as being an element of the $\mathcal{W}_{3}$ algebra, the charge $(3.15)$ is the pairing between $\mathcal{W}_{3}$ and its dual space. Accordingly, $(p, \rho)$ may be seen as a coadjoint vector of the $\mathcal{W}_{3}$ algebra. Its infinitesimal transformation law is given by [24]

$$
\begin{aligned}
\delta_{(X, \xi)} p=X p^{\prime}+2 X^{\prime} p-\frac{c}{12} X^{\prime \prime \prime}+2 \xi \rho^{\prime}+3 \xi^{\prime} \rho & \\
\delta_{(X, \xi)} \rho=X \rho^{\prime}+3 X^{\prime} \rho+\frac{\sigma}{3}[ & -2 \xi p^{\prime \prime \prime}-9 \xi^{\prime} p^{\prime \prime}-15 \xi^{\prime \prime} p^{\prime}-10 \xi^{\prime \prime \prime} p+ \\
& \left.+\frac{c}{12} \xi^{(5)}+\frac{192}{c}\left(\xi p p^{\prime}+\xi^{\prime} p^{2}\right)\right],
\end{aligned}
$$

where $\sigma$ is an irrelevant normalisation factor, prime denotes differentiation with respect to $x^{+}$, and $c=3 \ell / 2 G$ is the Brown-Henneaux central charge [22] (with $\ell$ the AdS radius). The infinitesimal transformations generated by $X$ imply that $p$ is a quasi-primary field with weight 2 with respect to conformal transformations, while $\rho$ is a primary field with weight 3. Together with the surface charges (3.15), these transformation laws yield the Poisson bracket

$$
\left\{Q_{(X, \xi)}[p, \pi], Q_{(Y, \zeta)}[p, \pi]\right\}=-\delta_{(X, \xi)} Q_{(Y, \zeta)}[p, \pi],
$$

which coincides with the non-linear bracket of a $\mathcal{W}_{3}$ algebra with central charge $c$. Similar considerations apply to models including fields with spin ranging from 2 to $N[24,26]$. The resulting asymptotic symmetry algebra is the direct sum of two copies of $\mathcal{W}_{N}$.

\footnotetext{
${ }^{6} \operatorname{In} \mathrm{AdS}_{3}$ this setup can be described by an $\mathfrak{s l}(N, \mathbb{R}) \oplus \mathfrak{s l}(N, \mathbb{R})$ Chern-Simons action with a principally embedded $\mathfrak{s l}(2, \mathbb{R}) \oplus \mathfrak{s l}(2, \mathbb{R})$ gravitational subalgebra.
} 
Flat $\mathcal{W}_{\mathbf{3}}$ algebra. The asymptotic symmetries of higher-spin theories at null infinity in three-dimensional flat space were discussed in [30,31, 38]. For the model describing the gravitational coupling of a field of spin $3,{ }^{7}$ it was found that symmetry transformations are labelled by four arbitrary, $2 \pi$-periodic functions $X(\varphi), \xi(\varphi), \alpha(\varphi)$ and $a(\varphi)$ on the celestial circle at null infinity. Of these, $X(\varphi)$ and $\alpha(\varphi)$ generate standard $\mathrm{BMS}_{3}$ superrotations and supertranslations (respectively), while $\xi$ and $a$ are their higher-spin extensions. The corresponding surface charges read

$$
Q_{(X, \xi, \alpha, a)}[j, \kappa, p, \rho]=\frac{1}{2 \pi} \int_{0}^{2 \pi} d \varphi[X(\varphi) j(\varphi)+\xi(\varphi) \kappa(\varphi)+\alpha(\varphi) p(\varphi)+a(\varphi) \rho(\varphi)],
$$

where the $2 \pi$-periodic functions $j, \kappa, p$ and $\rho$ determine a solution of the equations of motion. $p(\varphi)$ is the standard Bondi mass aspect (supermomentum), while $j(\varphi)$ is the angular momentum aspect (angular supermomentum); the functions $\rho$ and $\kappa$ generalise these quantities for a spin-3 field. As in the AdS case, the quadruple $(j, \kappa, p, \rho)$ may be seen as an element of the dual space of the asymptotic symmetry algebra. In particular, the higher-spin supermomentum $(p, \rho)$ transforms under higher-spin superrotations $(X, \xi)$ as a coadjoint vector of the $\mathcal{W}_{3}$ algebra, that is, according to (3.16), albeit with a central charge $c_{2}=3 / G$ instead of $c=3 \ell / 2 G$.

Inspection of the Poisson brackets satisfied by the surface charges (3.18), as displayed for instance in $[30,31]$, reveals that, in analogy with (3.5), the asymptotic symmetry algebra is a semi-direct sum

$$
\text { "flat } \mathcal{W}_{3} \text { algebra" } \equiv \mathcal{F} \mathcal{W}_{3}=\mathcal{W}_{3} \oplus_{\mathrm{Ad}}\left(\mathcal{W}_{3}\right)_{\mathrm{Ab}},
$$

where $\mathcal{W}_{3}$ is the standard $\mathcal{W}_{3}$ algebra and $\left(\mathcal{W}_{3}\right)_{\text {Ab }}$ denotes an Abelian Lie algebra isomorphic, as a vector space, to $\mathcal{W}_{3}$. This algebra is centrally extended, as the bracket between generators of $\mathcal{W}_{3}$ and those of $\left(\mathcal{W}_{3}\right)_{\mathrm{Ab}}$ includes a central charge $c_{2}$.

Induced representations, unitarity and characters. Since the flat $\mathcal{W}_{3}$ algebra (3.19) has the form $\mathfrak{g} \in \mathfrak{g}_{\mathrm{Ab}}$, with $\mathfrak{g}$ the standard $\mathcal{W}_{3}$ algebra, its unitary representations should be induced representations labelled by orbits of supermomenta under the coadjoint action of elements of a groupoid whose differentiation gives $\mathcal{W}_{3}$. However, the non-linearities that appear in $\mathcal{W}$ algebras make this step subtle. In the cases where the definition of the group is under control, as for $\mathrm{BMS}_{3}$, acting with group elements is required to specify the finite transformation of the supermomenta. This characterises the full orbit on which to define the wavefunctions that give a basis of the Hilbert space of each representation. Fortunately, one can bypass the need to control the group as follows. Generic $\mathcal{W}$ algebras define a Poisson manifold through (3.17) and one can classify the submanifolds on which the Poisson structure is invertible, called symplectic leaves [66]. In the case of the Virasoro algebra (which corresponds to the $\mathcal{W}_{N}$ algebra with $N=2$ ) this concept coincides with that of a coadjoint orbit of the Virasoro group. We thus propose to build unitary representations of

\footnotetext{
${ }^{7}$ Its action can be still written in a Chern-Simons form, with the gauge algebra $\mathfrak{s l}(3, \mathbb{R}) \notin(\mathfrak{s l}(3, \mathbb{R}))_{\mathrm{Ab}}$. The latter can be obtained as the İnönü-Wigner contraction of the $\mathfrak{s l}(3, \mathbb{R}) \oplus \mathfrak{s l}(3, \mathbb{R})$ gauge algebra of the corresponding model in $\mathrm{AdS}_{3}$ [30].
} 
flat $\mathcal{W}_{N}$ algebras as Hilbert spaces of wavefunctions defined on their symplectic leaves, on which we assume that one can define a suitable (quasi-invariant) measure. (See e.g. [68] for the construction of such a measure in the case of the Virasoro group.) One can make the analogy between symplectic leaves and coadjoint orbits even stronger: symplectic leaves of $\mathcal{W}_{N}$ algebras can be obtained as intersections of the coadjoint orbits of $\mathfrak{s l}(N)$-Kac Moody algebras with the constraints that implement the Hamiltonian reduction to $\mathcal{W}_{N}$ algebras [67].

A complete classification of the symplectic leaves of the standard $\mathcal{W}_{3}$ algebra has been worked out in $[66,67]$ and, according to our proposal, this provides the basis for a complete classification of irreducible, unitary representations of the flat $\mathcal{W}_{3}$ algebra. Here, following [59], we restrict instead our analysis to orbits of constant supermomenta, which can be classified from the infinitesimal transformation laws (3.16) given by the algebra. To describe the orbits of constant supermomenta let us pick a pair $(p, \rho)$ where $p(\varphi)=p_{0}$ and $\rho(\varphi)=\rho_{0}$ are constants, and act on it with an infinitesimal higher-spin superrotation $(X, \xi)$. Then, all terms involving derivatives of $p$ or $\rho$ in the transformation law (3.16) vanish, and we find

$$
\begin{aligned}
& \delta_{(X, \xi)} p_{0}=2 X^{\prime} p_{0}-\frac{c_{2}}{12} X^{\prime \prime \prime}+3 \xi^{\prime} \rho_{0}, \\
& \delta_{(X, \xi)} \rho_{0}=3 X^{\prime} \rho_{0}+\frac{\sigma}{3}\left[-10 \xi^{\prime \prime \prime} p_{0}+\frac{c_{2}}{12} \xi^{(5)}+\frac{192}{c_{2}} \xi^{\prime} p_{0}^{2}\right] .
\end{aligned}
$$

The little group for $\left(p_{0}, \rho_{0}\right)$ consists of higher-spin superrotations leaving it invariant. The little algebra is therefore spanned by pairs $(X, \xi)$ such that the right-hand sides of eqs. (3.20) vanish:

$$
\begin{aligned}
2 X^{\prime} p_{0}-\frac{c_{2}}{12} X^{\prime \prime \prime}+3 \xi^{\prime} \rho_{0} & =0, \\
3 X^{\prime} \rho_{0}+\frac{\sigma}{3}\left[-10 \xi^{\prime \prime \prime} p_{0}+\frac{c_{2}}{12} \xi^{(5)}+\frac{192}{c_{2}} \xi^{\prime} p_{0}^{2}\right] & =0 .
\end{aligned}
$$

The solutions of these equations depend on the values of $p_{0}$ and $\rho_{0}$. Here we will take $\rho_{0}=0$ for simplicity, i.e. we only consider cases where all higher-spin charges are switched off. Then, given $p_{0}$, eqs. (3.21) become two decoupled differential equations for the functions $X(\varphi)$ and $\xi(\varphi)$, leading to three different cases:

- For generic values of $p_{0}$, the only pairs $(X, \xi)$ leaving $\left(p_{0}, 0\right)$ invariant are constants, and generate a little group $\mathrm{U}(1) \times \mathbb{R}$.

- For $p_{0}=-n^{2} c_{2} / 96$ where $n$ is a positive odd integer, the pairs $(X, \xi)$ leaving $\left(p_{0}, 0\right)$ invariant are of the form

$$
X(\varphi)=A, \quad \xi(\varphi)=B+C \cos (n \varphi)+D \sin (n \varphi),
$$

where $A, B, C$ and $D$ are real numbers. The corresponding little group is the $n$-fold cover of $\mathrm{GL}(2, \mathbb{R})$.

- For $p_{0}=-n^{2} c_{2} / 24=-(2 n)^{2} c_{2} / 96$ where $n$ is a positive integer, the Lie algebra of the little group is spanned by

$$
\begin{aligned}
X(\varphi) & =A+B \cos (n \varphi)+C \sin (n \varphi), \\
\xi(\varphi) & =D+E \cos (n \varphi)+F \sin (n \varphi)+G \cos (2 n \varphi)+H \sin (2 n \varphi),
\end{aligned}
$$


where $A, B, \ldots, H$ are real coefficients. The little group is thus an $n$-fold cover of $\mathrm{SL}(3, \mathbb{R})$. In particular, $p_{0}=-c_{2} / 24$ realises the absolute minimum of energy among all supermomenta belonging to orbits with energy bounded from below. It is thus the supermomentum of the vacuum state, and indeed, upon using $c_{2}=3 / G$, the field configuration that corresponds to it is the metric of Minkowski space (with the spin-3 field set to zero on account of $\rho_{0}=0$ ).

The previous information on little groups is actually sufficient to evaluate certain characters along the lines of [49]. For instance, consider an induced module based on the orbit of a generic pair $\left(p_{0}, 0\right)$, and call $(s, \sigma)$ the spins of the representation $\mathcal{R}$ of the little group $\mathrm{U}(1) \times \mathbb{R}$. Then take a superrotation which is an element of the $\mathrm{U}(1)$ subalgebra (i.e. a rotation $f(\varphi)=\varphi+\theta$ ), and whose higher-spin supertranslation is an arbitrary combination $(\alpha(\varphi), a(\varphi))$. The only point on the orbit that is left invariant by the rotation is $\left(p_{0}, 0\right)$, and the whole integral over the orbit in (2.51) localises to that point. Therefore, in analogy with the $\mathrm{BMS}_{3}$ example, the detailed knowledge of the orbit is irrelevant to compute the character. In particular, the only components of $\alpha(\varphi)$ and $a(\varphi)$ that survive the integration are their zero-modes $\alpha^{0}$ and $a^{0}$, and the character takes the form

$$
\chi\left[\left(\operatorname{rot}_{\theta}, \alpha, a\right)\right]=e^{i s \theta} e^{i p_{0} \alpha^{0}} \int_{\mathcal{O}_{p_{0}}} \mathcal{D} \mu(q) \delta\left(q, \operatorname{rot}_{\theta} \cdot q\right) .
$$

In writing this we assumed the existence of a (quasi-invariant) measure $\mu$ on the orbit, whose precise expression is unimportant since different measures give representations that are unitarily equivalent [49]. We have manifested that the little group character reduces to $e^{i s \theta}$, so that this expression is an infinite-dimensional counterpart of (2.52). Our remaining task is to integrate the delta function. To do so, we use local coordinates on the orbit, which we choose to be the Fourier modes of higher-spin supermomenta in analogy with (2.53). Because $p_{0}$ is generic, the non-redundant coordinates on the orbit are the non-zero modes. The integral is thus

$$
\int_{\mathcal{O}_{p_{0}}} \mathcal{D} \mu(q) \delta\left(q, \operatorname{rot}_{\theta} \cdot q\right)=\prod_{n \in \mathbb{Z}^{*}}\left(\int d q_{n} \delta\left(q_{n}-e^{i n \theta} q_{n}\right)\right) \prod_{m \in \mathbb{Z}^{*}}\left(\int d \rho_{m} \delta\left(\rho_{m}-e^{i m \theta} \rho_{m}\right)\right),
$$

where we call $q_{n}$ the Fourier modes of the standard (spin 2) supermomentum, while $\rho_{m}$ are the modes of its higher-spin counterpart. Performing the integrals over Fourier modes and adding small imaginary parts $i \epsilon$ to $\theta$ to ensure convergence of the character, one obtains

$$
\chi\left[\left(\operatorname{rot}_{\theta}, \alpha, a\right)\right]=e^{i s \theta} e^{i p_{0} \alpha^{0}}\left(\prod_{n=1}^{\infty} \frac{1}{\left|1-e^{i n(\theta+i \epsilon)}\right|^{2}}\right)^{2} .
$$

This is a natural spin-3 extension of the spin-2 $\left(\mathrm{BMS}_{3}\right)$ massive character (3.2).

A similar computation can be performed for orbits of other higher-spin supermomenta $\left(p_{0}, 0\right)$. The only subtlety is that, for the values of $p_{0}$ for which the little group is larger than $\mathrm{U}(1) \times \mathbb{R}$, the orbit has higher codimension in $\mathcal{W}_{3}^{*}$ than the generic orbit we just discussed. Accordingly, there are fewer coordinates on the orbit and the products of integrals (3.25) 
are truncated. For instance, when $p_{0}=-n^{2} c / 24$ with $n$ a positive integer, the little group is generated by pairs $(X, \xi)$ of the form $(3.23)$, so that the Fourier modes providing nonredundant local coordinates on the orbit (in a neighbourhood of $\left(p_{0}, 0\right)$ ) are the modes $q_{m}$ with $m \notin\{-n, 0, n\}$ and the higher-spin modes $\rho_{m}$ with $m \notin\{-2 n,-n, 0, n, 2 n\}$. Assuming that the representation $\mathcal{R}$ of the little group is trivial, this produces a character

$$
\chi\left[\left(\operatorname{rot}_{\theta}, \alpha, a\right)\right]=e^{-i n^{2} c_{2} \alpha^{0} / 24}\left(\prod_{\substack{m=1, m \neq n}}^{\infty} \frac{1}{\left|1-e^{i m(\theta+i \epsilon)}\right|^{2}}\right) \cdot\left(\prod_{\substack{m=1, m \neq n, m \neq 2 n}}^{\infty} \frac{1}{\left|1-e^{i m(\theta+i \epsilon)}\right|^{2}}\right) .
$$

The choice $n=1$ specifies the vacuum representation of the flat $\mathcal{F} \mathcal{W}_{3}$ algebra; taking $\alpha$ to be a Euclidean time translation by $i \beta$, we get

$$
\chi_{\mathrm{vac}}\left[\left(\operatorname{rot}_{\theta}, \alpha=i \beta, a=0\right)\right]=e^{\beta c_{2} / 24}\left(\prod_{n=2}^{\infty} \frac{1}{\left|1-e^{i n(\theta+i \epsilon)}\right|^{2}}\right) \cdot\left(\prod_{n=3}^{\infty} \frac{1}{\left|1-e^{i n(\theta+i \epsilon)}\right|^{2}}\right) .
$$

Comparing with eq. (2.31), we recognise the product of the rotating one-loop partition functions of massless fields with spins two and three in three-dimensional flat space, including the classical piece $S^{(0)}=-\beta c_{2} / 24{ }^{8}$ This is one of our key results, that provides a first non-trivial check of our proposal to construct unitary representations of flat $\mathcal{W}_{N}$ algebras.

All the induced representations described above are unitary by construction, provided one can define (quasi-invariant) measures on the corresponding orbits. In analogy with representations of the $\mathfrak{b m s}_{3}$ algebra, they can also be described in terms of induced modules that generalise those discussed on page 20. Accordingly, one can again define a rest-frame state as one that is annihilated by all non-zero Fourier modes of the supertranslation generators $p$ and $\rho$ introduced in (3.18). Boosted states are obtained by acting with all Fourier modes of the superrotation generators $j$ and $\kappa$. Our representations thus evade the no-go theorems of [38] that stated the absence of unitary representations of the algebra (3.19) under certain conditions. The reason is that the representations considered in [38] are higher spin generalisations of those described in (3.14), and as such required $c_{2}=0$. Since some of the non-linear terms in (3.20b) depend on inverse powers of $c_{2}$ one had to first properly rescale some of the generators before taking $c_{2} \rightarrow 0$, which in turn rendered all higher-spin excitations to be null states, thus resulting in unitary representations of $\mathcal{F W}_{3}$ without higher-spin states. This argument, however, does not apply to the induced representations considered in this paper as these representations are unitary and allow for $c_{2} \neq 0$ without rendering all the higher-spin states unphysical.

Flat $\mathcal{W}_{N}$ algebras. The considerations of the previous pages can be generalised to higher-spin theories in flat space with spins ranging from 2 to $N$. In $\mathrm{AdS}_{3}$ the asymptotic symmetries of models with this field content are given by two copies of a $\mathcal{W}_{N}$ algebra and it is natural to anticipate that the corresponding theory in flat space $N$ will have an

\footnotetext{
${ }^{8}$ The value of the classical contribution depends on the normalisation of the Bondi mass. For example, if the normalisation is chosen in such a way that the mass of Minkowski space-time vanishes, then the corresponding classical action would vanish, as would the exponential prefactor of the character.
} 
asymptotic symmetry algebra

$$
\text { "flat } \mathcal{W}_{N} \text { algebra" } \equiv \mathcal{F} \mathcal{W}_{N}=\mathcal{W}_{N} \oplus_{\mathrm{Ad}}\left(\mathcal{W}_{N}\right)_{\mathrm{Ab}}
$$

in analogy with (3.5) and (3.19). The surface charges generating these symmetries should coincide with the pairing of the Lie algebra of (3.29) with its dual space, and they should satisfy a centrally extended algebra. Since the presence of higher-spin fields does not affect the value of the central charge in three-dimensional AdS gravity [23, 24], we expect the central charge in this case to be the usual $c_{2}=3 / G$ appearing in mixed brackets [28]. This structure was indeed observed for $N=4$ in [38]. We will now argue that this proposal must hold for any $N$ by showing that the vacuum character of (3.29), computed along the lines followed above for $\mathcal{F W}_{3}$, reproduces the product of one-loop partition functions of fields of spin $2,3, \ldots, N$.

According to our proposal for the characterisation of the representations of semi-direct sums of the type (3.29), unitary representation of flat $\mathcal{W}_{N}$ algebras are classified by their symplectic leaves, that is, by orbits of higher-spin supermomenta $\left(p_{1}, \ldots, p_{N-1}\right)$. (Here $p_{1}(\varphi)$ is the supermomentum that we used to write as $p(\varphi)$, while $p_{2}(\varphi)$ is what we called $\rho(\varphi)$ for $N=3$.) The infinitesimal transformations that generalise (3.16) and that define these orbits locally can be found e.g. in [26]. Here we focus on the vacuum orbit where we set all higher-spin charges to zero and take only $p_{1}=-c_{2} / 24$ to be non-vanishing. This particular supermomentum is left fixed by higher-spin asymptotic symmetries of the form

$$
X_{i}(\varphi)=A_{i}+\sum_{j=1}^{i}\left(B_{i j} \cos (j \varphi)+C_{i j} \sin (j \varphi)\right), \quad i=1, \ldots, N-1,
$$

where the coefficients $A_{i}, B_{i j}, C_{i j}$ are real. In principle, one can obtain such symmetry generators by looking for the stabiliser of the vacuum as in (3.21), using for instance the explicit formulas of [26]. However, a much simpler way to derive the same result is to look for the higher-spin isometries of the vacuum in the Chern-Simons formulation of the dynamics, in which models with fields of spin ranging from 2 to $N$ are described in flat space by a Chern-Simons action with gauge algebra $\mathfrak{s l}(N, \mathbb{R}) \in(\mathfrak{s l}(N, \mathbb{R}))_{\mathrm{Ab}}($ see e.g. $[18,31,38])$. In retarded Bondi coordinates $(r, u, \varphi)$, the vacuum field configuration takes the form

$$
A_{\mu}(x)=b(r)^{-1} g(u, \varphi)^{-1} \partial_{\mu}[g(u, \varphi) b(r)], \quad b(r)=\exp \left[\frac{r}{2} P_{-1}\right],
$$

where $g(u, \varphi)$ is the $\mathrm{SL}(N, \mathbb{R}) \ltimes \mathfrak{s l}(N, \mathbb{R})$ valued field given by

$$
g(u, \varphi)=\exp \left[\left(P_{1}+\frac{1}{4} P_{-1}\right) u+\left(J_{1}+\frac{1}{4} J_{-1}\right) \varphi\right]
$$

in terms of generators of the Poincaré algebra satisfying the commutation relations (3.4) with $m, n=-1,0,1$ (and of course without central extensions). The isometries of this field configuration are generated by gauge parameters of the form $(g \cdot b)^{-1} T_{a}(g \cdot b)$, where $T_{a}$ is any of the basis elements of the gauge algebra. Upon expanding $g^{-1} T_{a} g$ as a positiondependent linear combination of gauge algebra generators, the function multiplying the 
lowest weight generator coincides with the corresponding asymptotic symmetry parameter (see e.g. [24] for more details). The latter can be obtained as follows.

For convenience, let us diagonalise the Lorentz piece of the group element (3.32) as

$$
\exp \left[\left(J_{1}+\frac{1}{4} J_{-1}\right) \varphi\right]=B e^{i J_{0} \varphi} B^{-1}
$$

where $B$ is some $\operatorname{SL}(2, \mathbb{R})$ matrix. Then the gauge parameters generating the little group of the vacuum configuration can be written as

$$
\begin{gathered}
\exp \left[-\left(J_{1}+\frac{1}{4} J_{-1}\right) \varphi\right] \sum_{m=-\ell}^{\ell} \alpha^{m} W_{m}^{(\ell)} \exp \left[\left(J_{1}+\frac{1}{4} J_{-1}\right) \varphi\right] \\
=B e^{-i J_{0} \varphi} \sum_{m=-\ell}^{\ell} \alpha^{m} B^{-1} W_{m}^{(\ell)} B e^{i J_{0} \varphi} B^{-1}
\end{gathered}
$$

where the $\alpha^{m}$ 's are certain real coefficients, while the $W_{m}^{(\ell)}$ (with $2 \leq \ell \leq N$ and $-\ell \leq m \leq$ $\ell)$ are the generators of the $\mathfrak{s l}(N, \mathbb{R})$ algebra (including the $\left.J_{m} \equiv W_{m}^{(2)}\right)$. Note that the matrix $B$ preserves the conformal weight since it is an exponential of $\mathfrak{s l}(2, \mathbb{R})$ generators, so that

$$
\sum_{m=-\ell}^{\ell} \alpha^{m} B W_{m}^{(\ell)} B^{-1}=\sum_{m=-\ell}^{\ell} \tilde{\alpha}^{m} W_{m}^{(\ell)}
$$

for some coefficients $\tilde{\alpha}^{j}$ obtained by acting on the $\alpha^{m}$ 's with an invertible linear map. Because each generator $W_{m}^{(\ell)}$ has weight $m$ under $J_{0}$, expression (3.34b) can be rewritten as

$$
\sum_{m=-\ell}^{\ell} e^{i m \varphi} \tilde{\alpha}^{m} B W_{m}^{(\ell)} B^{-1}=\sum_{m, n=-\ell}^{\ell} \beta^{m n} W_{n}^{(\ell)} e^{i j \varphi}=\sum_{m=-\ell}^{\ell} e^{i m \varphi} \beta^{m \ell} W_{\ell}^{(\ell)}+\cdots
$$

for some coefficients $\beta^{m n}$. In the last step we omitted all terms proportional to $W_{m}^{(\ell)}$ 's with $m<\ell$; the important piece is the term that multiplies the highest-weight generator $W_{\ell}^{(\ell)}$ : it is the function on the circle that generates the asymptotic symmetry corresponding to the generator $\sum_{m=-\ell}^{\ell} \alpha^{m} W_{m}^{(\ell)}$ that we started with in (3.34a). Since the $\beta^{m \ell}$ 's are related to the $\alpha^{m}$ 's by an invertible linear map, and since there are $2 \ell+1$ linearly independent generators of this type, the isometries of the vacuum exactly span the set of functions of the form (3.30). This is what we wanted to prove; there are $N^{2}-1$ linearly independent asymptotic symmetry generators of this form, and they span the Lie algebra of $\operatorname{SL}(N, \mathbb{R})$.

The character associated with the vacuum representation of (3.29) can then be worked out exactly as in the cases $N=2$ and $N=3$ discussed above: using the Fourier modes of the $N-1$ components of supermomentum as coordinates on the orbit, we need to mod out the redundant modes. For the vacuum orbit, these are the modes ranging from $-\ell$ to $\ell$ for the $\ell^{\text {th }}$ component. The integral over the localising delta function in the Frobenius formula (2.51) then produces a character

$$
\chi\left[\left(\operatorname{rot}_{\theta}, a_{1}=i \beta, a_{2}=\ldots=a_{N-1}=0\right)\right]=e^{\beta c_{2} / 24} \prod_{s=2}^{N}\left(\prod_{n=s}^{\infty} \frac{1}{\mid 1-e^{\left.i n(\theta+i \epsilon)\right|^{2}}}\right) .
$$


Comparing with (2.31), we recognise the product of one-loop partition functions of massless higher-spin fields with spins ranging from 2 to $N$, including a classical contribution. This result confirms, on the one hand, our conjecture (3.29) for the asymptotic symmetry algebras of a generic higher-spin theory in three-dimensional flat space, and on the other hand it provides another consistency check of our proposal for the characterisations of the unitary representations of flat $\mathcal{W}_{N}$ algebras.

\subsection{Supersymmetry and super $\mathrm{BMS}_{3}$ characters}

The supersymmetric $\mathrm{BMS}_{3}$ groups describe the symmetries of three-dimensional, asymptotically flat supergravity [32, 69-72]. Here we exhibit the classification of unitary representations of their $\mathcal{N}=1$ version and show that the corresponding vacuum character coincides (in the Neveu-Schwarz sector) with the one-loop partition function of $\mathcal{N}=1$ supergravity. We then extend the matching between vacuum characters and one-loop partition functions to hypergravity theories, describing the gravitational coupling of a massless field of spin $s+1 / 2$. We start by reviewing briefly unitary representations of supersymmetric semi-direct products, referring to $[73,74]$ for details.

Supersymmetric induced representations. A super Lie group is a pair $\left(\Gamma_{0}, \gamma\right)$ where $\Gamma_{0}$ is a Lie group in the standard sense, while $\gamma$ is a super Lie algebra whose even part coincides with the Lie algebra of $\Gamma_{0}$, and whose odd part is a $\Gamma_{0}$-module such that the differential of the $\Gamma_{0}$ action be the bracket between even and odd elements of $\gamma$ [75]. Then a super semi-direct product is a super Lie group of the form [73, 74]

$$
\left(G \ltimes_{\sigma} A, \mathfrak{g} \notin(A+\mathcal{A})\right),
$$

where $G \ltimes A$ is a standard (bosonic) semi-direct product group with Lie algebra $\mathfrak{g} \in A$, and $\mathfrak{g} \in(A+\mathcal{A})$ is a super Lie algebra whose odd subalgebra $\mathcal{A}$ is a $G$-module such that the bracket between elements of $\mathfrak{g}$ and elements of $\mathcal{A}$ be the differential of the action of $G$ on $\mathcal{A}$, and such that $[A, \mathcal{A}]=0$ and $\{\mathcal{A}, \mathcal{A}\} \subseteq A$. By virtue of this definition, the action $\phi$ of $G$ on $\mathcal{A}$ is compatible with the super Lie bracket:

$$
\left\{\phi_{g} S, \phi_{g} T\right\}=\sigma_{g}\{S, T\} \quad \forall S, T \in \mathcal{A},
$$

where $\sigma$ is the action of $G$ on $A$.

It was shown in $[73,74]$ that all irreducible, unitary representations of a super semidirect product are induced in essentially the same sense as for standard, bosonic groups. In particular, they are classified by the orbits and little groups of $G \ltimes_{\sigma} A$. However, there are two important differences with respect to the purely bosonic case:

1. Unitarity rules out all orbits on which energy can be negative, so that the momentum orbits giving rise to unitary representations of the supergroup form a subset of the full menu of orbits available in the purely bosonic case. More precisely, given a momentum $p \in A^{*}$, it must be such that

$$
\langle p,\{S, S\}\rangle \geq 0 \quad \forall S \in \mathcal{A} .
$$


If this condition is not satisfied, the representations of (3.38) associated with the orbit $\mathcal{O}_{p}$ are not unitary. The momenta satisfying condition (3.40) are said to be admissible. Note that admissibility is a $G$-invariant statement: if $f \in G$ and if $p$ is admissible, then so is $f \cdot p$, by virtue of (3.39). For example, for Poincaré, the only admissible momenta are those of massive or massless particles with positive energy (and the trivial momentum $p=0$ ).

2. Given an admissible momentum $p$, the odd piece $\mathcal{A}$ of the supersymmetric translation algebra produces a (generally degenerate) Clifford algebra

$$
\mathcal{C}_{p}=T(\mathcal{A}) /\left\{S^{2}-\langle p,\{S, S\}\rangle \mid S \in \mathcal{A}\right\}
$$

where $T(\mathcal{A})$ is the tensor algebra of $\mathcal{A}$. Quotienting this algebra by its ideal generated by the radical of $\mathcal{A}$, one obtains a non-degenerate Clifford algebra $\overline{\mathcal{C}}_{p}$. Since $\mathcal{A}$ is a $G$-module, there exists an action of the little group $G_{p}$ on $\overline{\mathcal{C}}_{p}$; let us denote this action by $a \mapsto g \cdot a$ for $a \in \overline{\mathcal{C}}_{p}$ and $g \in G_{p}$. To obtain a representation of the full supergroup (3.38), one must find an irreducible representation $\tau$ of $\overline{\mathcal{C}}_{p}$ and a representation $\mathcal{R}_{0}$ of $G_{p}$ in the same space, that is compatible with $\tau$ in the sense that

$$
\tau[g \cdot a]=\mathcal{R}_{0}[g] \cdot \tau[a] \cdot\left(\mathcal{R}_{0}[g]\right)^{-1} .
$$

For finite-dimensional groups, the pair $\left(\tau, \mathcal{R}_{0}\right)$ turns out to be unique up to multiplication of $\mathcal{R}_{0}$ by a character of $G_{p}$ (and possibly up to parity-reversal). Given such a pair, we call it the fundamental representation of the supersymmetric little group.

The Clifford algebra (3.41) leads to a replacement of the irreducible, "spin" representations of the little group, by generally reducible representations $\mathcal{R}_{0} \otimes \mathcal{R}$. This is the multiplet structure of supersymmetry: the restriction of an irreducible unitary representation of a supergroup to its bosonic subgroup is generally reducible, and the various irreducible components account for the combination of spins that gives a SUSY multiplet. In the Poincaré group, an irreducible multiplet contains finitely many spins; by contrast, we will see below that a super-BMS $\mathrm{BM}_{3}$ multiplet contains infinitely many spins. Apart from this difference, the structure of induced representations of super semi-direct products is essentially the same as in the bosonic case: they consist of wavefunctions on an orbit, taking their values in the space of the representation $\mathcal{R}_{0} \otimes \mathcal{R}$. In particular, formula (2.51) for the character remains valid, up to the replacement of $\mathcal{R}$ by $\mathcal{R}_{0} \otimes \mathcal{R}$.

Supersymmetric $\mathbf{B M S}_{3}$ groups. Before turning to super $\mathrm{BMS}_{3}$, recall first that the $\mathcal{N}=1$ super Virasoro algebra is built by adding to $\operatorname{Vect}\left(S^{1}\right)$ an odd subalgebra $\mathcal{F}_{-1 / 2}\left(S^{1}\right)$ of $(-1 / 2)$-densities on the circle [76,77]. This produces a Lie superalgebra, isomorphic to $\operatorname{Vect}\left(S^{1}\right) \oplus \mathcal{F}_{-1 / 2}\left(S^{1}\right)$ as a vector space, which we will write as $\operatorname{sect}\left(S^{1}\right)$. Its elements are pairs $(X, S)$, where $X=X(\varphi) \partial / \partial \varphi$ and $S=S(\varphi)(d \varphi)^{-1 / 2}$, and the super Lie bracket is defined as

$$
[(X, S),(Y, T)] \equiv\left([X, Y]+S \otimes T, \phi_{X} T-\phi_{Y} S\right)
$$


Here $[X, Y]$ is the standard Lie bracket of vector fields and $\phi$ denotes the natural action of vector fields on $\mathcal{F}_{-1 / 2}\left(S^{1}\right)$, so that $\phi_{X} T$ is the $(-1 / 2)$-density whose component is

$$
X T^{\prime}-\frac{1}{2} X^{\prime} T
$$

Upon expanding the functions $X(\varphi)$ and $S(\varphi)$ in Fourier modes, one recovers the standard $\mathcal{N}=1$ supersymmetric extension of the Witt algebra. Choosing $S(\varphi)$ to be periodic or antiperiodic leads to the Ramond or the Neveu-Schwarz sector of the superalgebra, respectively.

The central extension of sVect $\left(S^{1}\right)$ is the super Virasoro algebra, svir. Its elements are triples $(X, S, \lambda)$ where $(X, S) \in \operatorname{sVect}\left(S^{1}\right)$ and $\lambda \in \mathbb{R}$, with a super Lie bracket defined as

$$
[(X, S, \lambda),(Y, T, \mu)\} \equiv\left([X, Y]+S \otimes T, \phi_{X} T-\phi_{Y} S, C(X, Y)+D(S, T)\right)
$$

where we write

$$
C(X, Y) \equiv-\frac{1}{48 \pi} \int_{0}^{2 \pi} d \varphi X Y^{\prime \prime \prime} \quad \text { and } \quad D(S, T) \equiv \frac{1}{12 \pi} \int_{0}^{2 \pi} d \varphi S^{\prime} T^{\prime} .
$$

Here $C$ is the standard Gelfand-Fuchs cocycle of the Virasoro algebra, and $C(X, Y)+$ $D(S, T)$ is its supersymmetric generalisation. Again, upon expanding the functions $X$ and $S$ in Fourier modes, one obtains the usual commutation relations of the $\mathcal{N}=1$ super Virasoro algebra, with the central charge $Z=(0,0,1)$.

We can now define the $\mathcal{N}=1$ super $\mathrm{BMS}_{3}$ group [69, 70]: it is a super semi-direct product (3.38) whose even piece is the $\mathrm{BMS}_{3}$ group (3.1), and whose odd subspace is $\mathcal{F}_{-1 / 2}\left(S^{1}\right)$ with the bracket $\{S, T\}=S \otimes T$. In other words, the (centerless) super $\mathfrak{b m s}_{3}$ algebra is a super semi-direct sum

$$
\mathfrak{s b m s}_{3}=\operatorname{Vect}\left(S^{1}\right) \notin\left(\operatorname{Vect}\left(S^{1}\right)_{\mathrm{Ab}} \oplus \mathcal{F}_{-1 / 2}\right),
$$

where $\operatorname{Vect}\left(S^{1}\right)_{\mathrm{Ab}} \oplus \mathcal{F}_{-1 / 2}$ may be seen as an Abelian version of $\operatorname{siect}\left(S^{1}\right)$. Again, choosing periodic/antiperiodic boundary conditions for $\mathcal{F}_{-1 / 2}$ yields the Ramond/Neveu-Schwarz sector of the theory (respectively). Upon including central extensions, elements of the (now centrally extended) super $\mathfrak{b m s}_{3}$ algebra become 5 -tuples $(X, \alpha, S ; \lambda, \mu)$, where $(X, \alpha, S)$ belongs to $\mathfrak{s b m s}_{3}$ and $\lambda, \mu$ are real numbers, with a super Lie bracket

$$
\begin{aligned}
& {[(X, \alpha, S ; \lambda, \mu),(Y, \beta, T ; \kappa, \nu)\}=} \\
& =\left([X, Y],[X, \beta]-[Y, \alpha], \phi_{X} T-\phi_{Y} S ; C(X, Y), C(X, \beta)-C(Y, \alpha)+D(S, T)\right),
\end{aligned}
$$

with $C(X, Y)$ and $D(S, T)$ written in (3.46). Upon expanding all fields in Fourier modes, one finds the brackets (3.4) where the $J_{m}$ 's and $P_{m}$ 's are modes of $X$ 's and $\alpha$ 's (respectively), supplemented with

$$
\begin{aligned}
{\left[J_{m}, Q_{r}\right] } & =\left(\frac{m}{2}-r\right) Q_{m+r} \\
{\left[P_{m}, Q_{r}\right] } & =0
\end{aligned}
$$




$$
\left\{Q_{r}, Q_{s}\right\}=P_{r+s}+\frac{c_{2}}{6} r^{2} \delta_{r+s, 0},
$$

where the supercharges $Q_{r}$ are the modes of $S$ 's. The indices $r, s$ are integers/half-integers in the Ramond/Neveu-Schwarz sector.

In the gravitational context, the functions $X$ and $\alpha$ generate superrotations and supertranslations, while $S(\varphi)$ generates local supersymmetry transformations that become global symmetries upon enforcing suitable boundary conditions on the fields. The surface charge associated with $(X, \alpha, S)$ then takes the form [69]

$$
Q_{(X, \alpha, S)}[j, p, \psi]=\frac{1}{2 \pi} \int_{0}^{2 \pi} d \varphi[X(\varphi) j(\varphi)+\alpha(\varphi) p(\varphi)+S(\varphi) \psi(\varphi)],
$$

where $j$ and $p$ are the angular momentum and Bondi mass aspects that we already encountered in (3.18), while $\psi(\varphi)$ is one of the subleading components of the gravitino at null infinity. Upon using formula (3.17), these charges satisfy the algebra (3.48)-(3.49) with $c_{2}=$ $3 / G$. Note that the gravitino naturally satisfies Neveu-Schwarz boundary conditions on the celestial circles, as it transforms under a projective representation of the Lorentz group.

The construction of the super $\mathrm{BMS}_{3}$ group can be generalised in a straightforward way. Indeed, let $G$ be a (bosonic) group, $\mathfrak{g}$ its Lie algebra, $\mathfrak{s g}$ a super Lie algebra whose even subalgebra is $\mathfrak{g}$. Then one can associate with $G$ a (bosonic) semi-direct product $G \ltimes \mathfrak{g}-$ the even $\mathrm{BMS}_{3}$ group is of that form, with $G$ the Virasoro group. Now let $\mathfrak{s g}_{\mathrm{Ab}}$ denote the "Abelian" super Lie algebra which is isomorphic to $\mathfrak{s g}$ as a vector space, but where all brackets involving elements of $\mathfrak{g}$ are set to zero. One may then define a super semi-direct product

$$
\left(G \ltimes \mathfrak{g}, \mathfrak{g} \oplus \mathfrak{s g} \mathfrak{g b}_{\mathrm{Ab}}\right)
$$

where we use the notation (3.38). This structure appears to be ubiquitous in threedimensional, asymptotically flat supersymmetric higher-spin theories.

Unitary representations of the super $\mathrm{BMS}_{3}$ group can be classified along the lines briefly explained at the beginning of this subsection. In the remainder of this section we describe this classification in some more detail and use it to evaluate characters of the centrally extended super $\mathrm{BMS}_{3}$ group.

Admissible super $\mathbf{B M S}_{\mathbf{3}}$ orbits. The unitary representations of super $\mathrm{BMS}_{3}$ are classified by the same orbits as in the purely bosonic case. However, supermomenta that do not satisfy condition (3.40) are forbidden, so our first task is to understand which orbits are admissible. To begin, recall that the admissibility condition (3.40) is invariant under superrotations. Thus, if we consider a supermomentum orbit containing a constant $p_{0}$ say, the supermomenta on the orbit will be admissible if and only if $p_{0}$ is. Including the central charge $c_{2}$, we ask: which pairs $\left(p_{0}, c_{2}\right)$ are such that

$$
\left\langle\left(p_{0}, c_{2}\right),\{S, S\}\right\rangle \geq 0 \quad \forall S \in \mathcal{F}_{-1 / 2}\left(S^{1}\right) ?
$$

Here $\langle.,$.$\rangle is the pairing between supermomenta and supertranslations, given by the terms$ pairing $p$ and $\alpha$ in the surface charges (3.18) and (3.50). Using the super Lie bracket (3.48), we find

$$
\left\langle\left(p_{0}, c_{2}\right),\{S, S\}\right\rangle=\frac{1}{2 \pi} \int_{0}^{2 \pi} d \varphi\left(p_{0}(S(\varphi))^{2}+\frac{c_{2}}{6}\left(S^{\prime}(\varphi)\right)^{2}\right) .
$$


Since the term involving $\left(S^{\prime}\right)^{2}$ can be made arbitrarily large while keeping $S^{2}$ arbitrarily small, a necessary condition for $\left(p_{0}, c_{2}\right)$ to be admissible is that $c_{2}$ be non-negative. The admissibility condition on $p_{0}$, on the other hand, depends on the sector under consideration:

- In the Ramond sector, $S(\varphi)$ is a periodic function on the circle. In particular, $X(\varphi)=$ const is part of the supersymmetry algebra, so for expression (3.53) to be non-negative for any $S$, we must impose $p_{0} \geq 0$.

- In the Neveu-Schwarz sector, $S(\varphi)$ is antiperiodic (i.e. $S(\varphi+2 \pi)=-S(\varphi)$ ) and can be expanded in Fourier modes as

$$
S(\varphi)=\sum_{n \in \mathbb{Z}} s_{n+1 / 2} e^{i(n+1 / 2) \varphi} .
$$

Then expression (3.53) becomes

$$
\left\langle\left(p_{0}, c_{2}\right),\{S, S\}\right\rangle=\sum_{n \in \mathbb{Z}}\left[p_{0}+\frac{c_{2}}{6}(n+1 / 2)^{2}\right]\left|s_{n+1 / 2}\right|^{2},
$$

and the admissibility condition amounts to requiring all coefficients in this series to be non-negative, which gives

$$
p_{0} \geq-\frac{c_{2}}{24} .
$$

These bounds are consistent with earlier observations in three-dimensional supergravity [69], according to which Minkowski space-time (corresponding to $p_{0}=-c_{2} / 24$ ) realises the Neveu-Schwarz vacuum, while the Ramond vacuum is realised by the null orbifold (corresponding to $\left.p_{0}=0\right)$. Analogous results hold in $\mathrm{AdS}_{3}$ [78]. More general admissibility conditions can be worked out for non-constant supermomenta by adapting the proof of the positive energy theorem in [60]; we will address this question elsewhere.

Super $\mathbf{B M S}_{\boldsymbol{3}}$ multiplets. As explained around (3.41), a unitary representation of super $\mathrm{BMS}_{3}$ based on an orbit $\mathcal{O}_{p}$ comes equipped with a representation $\tau$ of the Clifford algebra

$$
\mathcal{C}_{p}=T\left(\mathcal{F}_{-1 / 2}\left(S^{1}\right)\right) /\left\{S^{2}-\left\langle\left(p, c_{2}\right),\{S, S\}\right\rangle\right\} .
$$

Let us build such a representation. We will work in the Neveu-Schwarz sector, and we take $p$ to be a constant admissible supermomentum $p_{0}=M-c_{2} / 24$ with $M>0$, whose little group is $\mathrm{U}(1)$. Then the bilinear form (3.55) is non-degenerate and the representation $\tau$ of the Clifford algebra (3.57) must be such that

$$
\tau\left[Q_{r}\right] \cdot \tau\left[Q_{s}\right]+\tau\left[Q_{s}\right] \cdot \tau\left[Q_{r}\right]=\left(\frac{c_{2}}{6}\left(r^{2}-1 / 4\right)+M\right) \delta_{r+s, 0}, \quad r, s \in \mathbb{Z}+1 / 2 .
$$

In order to make $\tau$ irreducible, we start with a highest-weight state $|0\rangle$ such that $\tau\left[Q_{r}\right]|0\rangle=0$ for $r>0$, and generate the space of the representation by its "descendants" $\tau\left[Q_{-r_{1}}\right] \ldots \tau\left[Q_{-r_{n}}\right]|0\rangle, r_{1}>\ldots>r_{n}>0$. It follows from the Lie brackets (3.49) that each descendant state has $\operatorname{spin} s+\sum_{i=1}^{n} r_{i}$, where $s$ is the spin of the state $|0\rangle$; this observation uniquely determines the little group representation $\mathcal{R}_{0}$ satisfying (3.42). Thus, a super $\mathrm{BMS}_{3}$ particle consists of infinitely many particles with spins increasing from $s$ to infinity. 
A similar construction can be carried out for the vacuum supermomentum at $M=0$, with the subtlety that the Clifford algebra (3.57) (or equivalently (3.58)) is degenerate. As explained below (3.41), one needs to quotient (3.57) by the radical of the bilinear form (3.55), resulting in a non-degenerate Clifford algebra $\overline{\mathcal{C}_{p}}$. In the case at hand this algebra is generated by supercharges $Q_{r}$ with $|r|>1$, and the representation $\tau$ must satisfy (3.58) with $M=0$ and $|r|,|s|>1$. The remainder of the construction is straightforward: starting from a state $|0\rangle$ with, say, vanishing spin, we generate the space of the representation by acting on it with $\tau\left[Q_{-r}\right]$ 's, where $r>1$. The vacuum representation of super $\mathrm{BMS}_{3}$ thus contains infinitely many "spinning vacua" with increasing spins.

Characters. The Fock space representations just described can be used to evaluate characters. For example, in the massive case we find

$$
\operatorname{tr}\left[e^{i \theta J_{0}}\right]=e^{i \theta s}\left[1+e^{i \theta / 2}+e^{3 i \theta / 2}+e^{2 i \theta}+\cdots\right]=e^{i \theta s} \prod_{n=1}^{\infty}\left(1+e^{i(n-1 / 2)(\theta+i \epsilon)}\right),
$$

where we have added a small imaginary part to $\theta$ to ensure convergence of the product; the trace is taken in the fermionic Fock space associated with the "highest-weight state" $|0\rangle$. The vacuum case is similar, except that the product would start at $n=2$ rather than $n=1$ (and $s=0$ ). Note that (3.59) explicitly breaks parity invariance; this can be fixed by replacing the parity-breaking Fock space representations $\tau$ described above by parity-invariant tensor products $\tau \otimes \bar{\tau}$, where $\bar{\tau}$ is the same as $\tau$ with the replacement of $Q_{r}$ by $Q_{-r}$. The trace of a rotation operator in the space of $\tau \otimes \bar{\tau}$ then involves the norm squared of the product appearing in (3.59).

As explained on page 31, the character of an induced representation of a super semidirect product takes the same form (2.51) as in the bosonic case, but with the character of $\mathcal{R}$ replaced by that of a (reducible) representation $\mathcal{R}_{0} \otimes \mathcal{R}$ compatible with the Clifford algebra representation $\tau$. We thus find that the character of a rotation by $\theta$ (together with a Euclidean time translation by $\beta$ ), in the parity-invariant vacuum representation of the $\mathcal{N}=1$, Neveu-Schwarz super $\mathrm{BMS}_{3}$ group, reads

$$
\begin{aligned}
\chi_{\text {vac }}^{\text {super BMS }}\left[\left(\operatorname{rot}_{\theta}, i \beta\right)\right] & =\chi_{\text {vac }}^{\mathrm{BMS}}\left[\left(\operatorname{rot}_{\theta}, i \beta\right)\right] \cdot \prod_{n=2}^{\infty}\left|1+e^{i(n-1 / 2)(\theta+i \epsilon)}\right|^{2} \\
& =e^{\beta c_{2} / 24} \prod_{n=2}^{\infty} \frac{\left|1+e^{i(n-1 / 2)(\theta+i \epsilon)}\right|^{2}}{\left|1-e^{i n(\theta+i \epsilon)}\right|^{2}}
\end{aligned}
$$

Comparing with (2.31) and (2.50), we recognise the product of the partition functions of two massless fields with spins 2 and $3 / 2$, that is, the one-loop partition function of $\mathcal{N}=1$ supergravity in three-dimensional flat space.

Higher-spin supersymmetry and hypergravity. In [32, 71], the authors considered a three-dimensional hypergravity theory consisting of a metric coupled to a single field with half-integer spin $s+1 / 2$, with $s$ larger than one. Upon imposing suitable asymptotically flat boundary conditions, they found that the asymptotic symmetry algebra spans a superalgebra that extends the bosonic $\mathfrak{b m s}_{3}$ algebra by generators $Q_{r}$ of spin $s+1 / 2$. 
The one-loop partition function of that system is the product of the graviton partition function (see eq. (2.31) for $s=2$ ) with the fermionic partition function (2.50). We now show that this partition function coincides with the vacuum character of the corresponding asymptotic symmetry group (in the Neveu-Schwarz sector).

The irreducible, unitary representations of the asymptotic symmetry group of [32] are classified by the same orbits and little groups as for the standard $\mathrm{BMS}_{3}$ group. In particular, we can consider the orbit of a constant supermomentum $p_{0}=M-c_{2} / 24$; the associated Clifford algebra representation $\tau$ mentioned below (3.41) then satisfies a natural generalization of eq. (3.58) (see eq. (7.23) in [32])

$$
\tau\left[Q_{r}\right] \tau\left[Q_{\ell}\right]+\tau\left[Q_{\ell}\right] \tau\left[Q_{r}\right]=\prod_{j=0}^{s-1}\left(\frac{c_{2}}{6}\left(r^{2}-\frac{(2 j+1)^{2}}{4}\right)+M\right) \delta_{r+\ell, 0}
$$

where $r$ and $\ell$ are integers or half-integers, depending on the sector under consideration (Ramond or Neveu-Schwarz, respectively). In order for the orbit to be admissible in the sense of (3.40), the value of $M$ must be chosen so as to ensure that all coefficients on the right-hand side of (3.61) are non-negative. In particular, the vacuum value $M=0$ is admissible in the Neveu-Schwarz sector, in which case the anticommutators $\left\{\tau\left[Q_{r}\right], \tau\left[Q_{-r}\right]\right\}$ vanish for $|r|=1 / 2, \ldots, s-1 / 2$. Thus, in the Neveu-Schwarz vacuum, the Clifford algebra (3.61) degenerates and $\tau$ must really be seen as a representation of the non-degenerate subalgebra generated by the $Q_{r}$ 's with $|r| \geq s$. The corresponding Fock space representation can be built as explained below (3.58), and the spins of the basis states in this representation are uniquely determined by the fact that the $Q_{r}$ 's have spin $s+1 / 2$. The corresponding Fock space character is thus

$$
\operatorname{tr}\left[e^{i \theta J_{0}}\right]=\prod_{n=s+1}^{\infty}\left(1+e^{i(n-1 / 2)(\theta+i \epsilon)}\right)
$$

which generalises (3.59). The character for $\tau \otimes \bar{\tau}$ is the squared norm of this expression, and the resulting vacuum character of the hypersymmetric $\mathrm{BMS}_{3}$ group is

$$
\chi_{\text {vac }}^{\text {hyper BMS }}\left[\left(\operatorname{rot}_{\theta}, i \beta\right)\right]=e^{\beta c_{2} / 24} \frac{\prod_{n=s}^{\infty}\left|1+e^{i(n+1 / 2)(\theta+i \epsilon)}\right|^{2}}{\prod_{m=2}^{\infty}\left|1-e^{i m(\theta+i \epsilon)}\right|^{2}} .
$$

As announced earlier, this coincides with the one-loop partition function of asymptotically flat gravity coupled to a massless field with spin $s+1 / 2$.

\section{Further directions}

A first, natural extension of our work will be to compute one-loop partition functions for the missing particles in flat space, i.e. mixed-symmetry and continuous spin particles. The first case corresponds to representations of the little groups $\mathrm{SO}(D-1)$ or $\mathrm{SO}(D-2)$ with arbitrary weights, and goes beyond our simplifying restriction to fully symmetric fields/weights 
of the form $(s, 0, \ldots, 0)$. The second case corresponds instead to generic massless particles, associated with representations of the full little group $\mathrm{SO}(D-2) \ltimes \mathbb{R}^{D-2}$. Both setups may be physically relevant: the vast majority of string excitations leads to mixed-symmetry massive fields, so that any comparison between string models and higher-spin (gauge) theories cannot forgo a good control over mixed symmetry particles. Continuous spin particles are instead more elusive. For a long time, following Wigner's intuition [50], they have been considered as unphysical. Recent analyses have instead provided indications that these particles may even evade the standard no-go arguments against higher-spin interactions [79]. In view of our discussion at the beginning of section 1, these representations are actually quite promising: in field theory they are realised by gauge theories [80]; nevertheless they intrinsically bring in a dimensionful parameter (e.g. the eigenvalue of the square of the PauliLubanski vector in $D=4$ ). In both cases one can easily compute the associated Poincaré characters with the techniques of section 2.4. Moreover, both Bose and Fermi mixed symmetry fields admit a Lagrangian description similar to the one we rely on in this paper (see e.g. $[81,82])$, so that we expect to be able to smoothly extend our considerations to this class of fields. A Lagrangian description of continuous-spin particles has also been proposed recently [80] and it will be interesting to test its structure by computing its one-loop partition function and comparing it with the Poincaré characters of continuous spin particles.

Another possible interesting application of our results will be to study carefully the flat-space limit of AdS partition functions. As we have discussed below (2.19) and in the final remark of section 2.4, it is not straightforward to recover partition functions in flat space and Poincaré characters as limits of partition functions in AdS [44, 83] and characters of the conformal algebra [57]. Therefore, even if considering this limit for free actions is trivial, the corresponding partition functions already give a feeling of the difficulties that become so dramatic when interactions are switched on. Clarifying how one can properly regularise the flat limit of one-loop partition functions may thus give insights on how to address this pathological limit in more general terms.

In three dimensions we also just started to scratch the surface of the representation theory of flat $\mathcal{W}$ algebras. It will be interesting to complete the classification of coadjoint orbits and to interpret the role of classes of representations characterised by different little groups. In addition, one has to systematise the construction of the Hilbert spaces of each representation, both via the wavefunction construction that is typical of the representations of the $\mathrm{BMS}_{3}$ group or via induced module constructions.

\section{Acknowledgments}

We are grateful to G. Barnich, T. Basile, X. Bekaert, N. Boulanger, D. Grumiller, A. Lepage-Jutier and P. Lowdon for illuminating discussions on closely related topics. The research of A.C. and H.A.G. was partially supported by the ERC Advanced Grant "SyDuGraM", by FNRS-Belgium (convention FRFC PDR T.1025.14 and convention IISN 4.4514.08) and by the "Communauté Française de Belgique" through the ARC program. The work of B.O. was supported by a doctoral fellowship of the Wiener-Anspach Foundation and by the Fund for Scientific Research-FNRS under grant number FC-95570. Fi- 
nally, this research of M.R. is supported by the FWF projects P27182-N27 and the START project Y435-N16. M.R. is also supported by a DOC fellowship of the Austrian Academy of Sciences, the Doktoratskolleg Particles and Interactions (FWF project DKW1252-N27) and the FWF project I 1030-N27.

\section{A From mixed traces to bosonic characters}

\section{A.1 Mixed traces and symmetric polynomials}

In this part of the appendix we prove that the mixed trace (2.20) of $\mathbb{I}_{\mu_{s}, \alpha_{s}}$ in $D$ dimensions coincides with a certain difference of complete homogeneous symmetric polynomials in the traces of $J^{n}$ as given by

$$
\chi_{s}[n \vec{\theta}]=h_{s}\left(J^{n}\right)-h_{s-2}\left(J^{n}\right),
$$

where

$$
h_{s}\left(J^{n}\right)=\sum_{\substack{m_{1}, \ldots, m_{s} \in \mathbb{N} \\ m_{1}+2 m_{2}+\ldots+s m_{s}=s}}\left[\prod_{k=1}^{s} \frac{\left(\operatorname{Tr}\left[\left(J^{n}\right)^{k}\right]\right)^{m_{k}}}{m_{k} ! k^{m_{k}}}\right] .
$$

By definition, the complete homogeneous symmetric polynomial of degree $s$ in $D$ complex variables $\lambda_{1}, \ldots, \lambda_{D}$ is

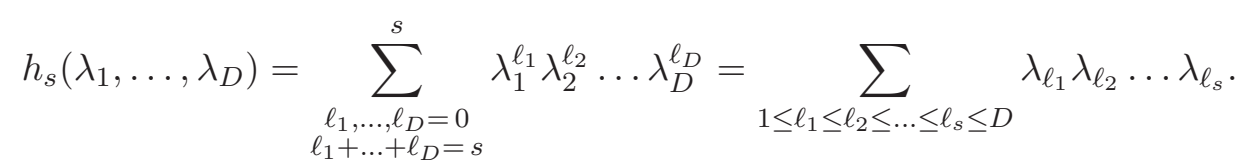

Using the variant of Newton's identities

$$
h_{s}\left(\lambda_{1}, \ldots, \lambda_{D}\right)=\frac{1}{s} \sum_{N=1}^{s} h_{s-N}\left(\lambda_{1}, \ldots, \lambda_{D}\right)\left(\lambda_{1}^{N}+\ldots+\lambda_{D}^{N}\right),
$$

one can show by recursion (see e.g. [84, p. 24f]) that the polynomial (A.3) can equivalently be written as in (A.2):

$$
h_{s}\left(\lambda_{1}, \ldots, \lambda_{D}\right)=\sum_{\substack{m_{1}, \ldots, m_{s} \in \mathbb{N} \\ m_{1}+2 m_{2}+\ldots+s m_{s}=s}} \prod_{k=1}^{s} \frac{\left(\lambda_{1}^{k}+\ldots+\lambda_{D}^{k}\right)^{m_{k}}}{m_{k} ! k^{m_{k}}} .
$$

We will use this relation later. To prove (A.1), we start with the following:

Lemma. Let $J$ be a complex $D \times D$ matrix with eigenvalues $\lambda_{1}, \ldots, \lambda_{D}$. Then,

$$
\left(\delta^{\mu \alpha}\right)^{s} \frac{1}{s !}\left(J_{\mu \alpha}\right)^{s}=h_{s}\left(\lambda_{1}, \lambda_{2}, \ldots, \lambda_{D}\right),
$$

where we use the same notation for contracting symmetrised indices as in (2.13). 
Proof. The left-hand side of (A.6) can be seen as a trace over symmetric tensor powers of $J$. Indeed, $\delta^{\mu \alpha} J_{\mu \alpha}=\operatorname{Tr}(J)$ is clear; as for $\frac{1}{2}\left(\delta^{\mu \alpha}\right)^{2}\left(J_{\mu \alpha}\right)^{2}$, one gets

$$
\frac{1}{2}\left(\delta^{\mu \alpha}\right)^{2}\left(J_{\mu \alpha}\right)^{2}=\frac{1}{2}\left(\operatorname{Tr}(J)^{2}+\operatorname{Tr}\left(J^{2}\right)\right)=\operatorname{Tr}\left(S^{2}(J)\right)=\frac{1}{2} \sum_{i=1}^{2} \operatorname{Tr}\left(J^{i}\right) \operatorname{Tr}\left(S^{2-i}(J)\right),
$$

where $S^{k}(J)$ denotes the $k^{\text {th }}$ symmetric tensor power of $J$. One can then define recursively

$$
\frac{1}{s !}\left(\delta^{\mu \alpha}\right)^{s}\left(J_{\mu \alpha}\right)^{s}=\operatorname{Tr}\left(S^{s}(J)\right)=\frac{1}{s} \sum_{i=1}^{s} \operatorname{Tr}\left(J^{i}\right) \operatorname{Tr}\left(S^{s-i}(J)\right)
$$

so that $\frac{1}{s !}\left(\delta^{\mu \alpha}\right)^{s}\left(J_{\mu \alpha}\right)^{s}$ is just a trace in the $s^{\text {th }}$ symmetric tensor power of the $D$ dimensional vector space $V$ on which $J_{\mu \alpha}$ acts as a linear operator. Now consider an eigenbasis $\left\{e_{1}, \ldots, e_{D}\right\}$ for $J_{\mu \alpha}$, with $J \cdot e_{k}=\lambda_{k} e_{k}$. Since $\frac{1}{s !}\left(J_{\mu \alpha}\right)^{s}$ is the $s^{\text {th }}$ symmetric tensor power of $J_{\mu \alpha}$ one can construct an eigenbasis for $\frac{1}{s !}\left(J_{\mu \alpha}\right)^{s}$ by symmetrising $e_{k_{1}} \otimes e_{k_{2}} \otimes \ldots \otimes e_{k_{D}}$, with $k_{1} \leq k_{2} \leq \ldots \leq k_{D}$. These eigenvectors have eigenvalues $\lambda_{l_{1}} \lambda_{l_{2}} \ldots \lambda_{l_{D}}$, and since $\left(\delta^{\mu \alpha}\right)^{s} \frac{1}{s !}\left(J_{\mu \alpha}\right)^{s}$ is the trace of $\frac{1}{s !}\left(J_{\mu \alpha}\right)^{s}$, relation (A.6) follows upon using the second expression of $h_{s}\left(\lambda_{1}, \ldots, \lambda_{D}\right)$ in (A.3).

We can now turn to the proof of (A.1). To this end we fix conventionally the number of terms entering the contraction of two symmetrised expressions as follows. Objects with lower indices are symmetrized with the minimum number of terms required and without overall normalisation factor, while objects with upper indices are not symmetrised at all, since the symmetrisation is induced by the contraction. This specification is needed because terms with lower and upper indices in a contraction may have a different index structure and therefore the number of terms needed for their symmetrisation may be different. For instance

$$
\begin{aligned}
& A^{\mu} B^{\mu} C^{\mu} D_{\mu \mu} E_{\mu} \equiv A^{\mu} B^{\nu} C^{\rho}\left(D_{\mu \nu} E_{\rho}+D_{\nu \rho} E_{\mu}+D_{\rho \mu} E_{\nu}\right) \\
& =\frac{1}{2}\left(A^{\mu} B^{\nu} C^{\rho}+A^{\nu} B^{\rho} C^{\mu}+A^{\rho} B^{\mu} C^{\nu}+A^{\mu} B^{\rho} C^{\nu}+A^{\rho} B^{\nu} C^{\mu}+A^{\nu} B^{\mu} C^{\rho}\right) D_{\mu \nu} E_{\rho} .
\end{aligned}
$$

In order to simplify computations, we define

$$
T_{\mu_{s}, \alpha_{s}} \equiv J_{\mu \alpha} \ldots J_{\mu \alpha}, \quad T^{[s]} \equiv T_{\mu_{s}, \alpha_{s}}\left(\delta^{\mu \alpha}\right)^{s},
$$

which implies the contraction rules

$$
\delta^{\mu \mu} T_{\mu_{s}, \alpha_{s}}=2 \delta_{\alpha \alpha} T_{\mu_{s-2}, \alpha_{s-2}}, \quad \delta^{\alpha \alpha} T_{\mu_{s}, \alpha_{s}}=2 \delta_{\mu \mu} T_{\mu_{s-2}, \alpha_{s-2}} .
$$

In terms of the tensors $T_{\mu_{s}, \alpha_{s}}$, the mixed trace (2.20) can be written as

$$
\begin{aligned}
& \chi_{s}[n \vec{\theta}]=\frac{1}{s !} T_{\mu_{s}, \beta_{s}}\left[\left(\delta^{\mu \beta}\right)^{s}+\sum_{m=1}^{\left\lfloor\frac{s}{2}\right\rfloor} \frac{(-1)^{m} s ![D+2(s-m-2)] ! !}{2^{m} m !(s-2 m) ![D+2(s-2)] ! !}\left(\delta^{\mu \mu}\right)^{m}\left(\delta^{\mu \beta}\right)^{s-2 m}\left(\delta^{\beta \beta}\right)^{m}\right] \\
& \stackrel{\text { A.11) }}{=} \frac{1}{s !} T^{[s]}+\sum_{m=1}^{\left[\frac{s}{2}\right]} \frac{(-1)^{m}[D+2(s-m-2)] ! !}{2^{m-1} m !(s-2 m) ![D+2(s-2)] ! !}\left(\delta^{\mu \mu}\right)^{m}\left(\delta^{\mu \beta}\right)^{s-2 m}\left(\delta^{\beta \beta}\right)^{m-1} \delta_{\mu \mu} T_{\mu_{s-2}, \beta_{s-2}} .
\end{aligned}
$$


To compute the trace of the $\left(\delta^{\mu \mu}\right)^{m}\left(\delta^{\mu \beta}\right)^{s-2 m}\left(\delta^{\beta \beta}\right)^{m-1}$ terms, we first change our symmetrisation from $\delta_{\mu \mu} T_{\mu_{s-2}, \beta_{s-2}}$ (which contains $\frac{s !}{2(s-2) !}$ terms) to the aforementioned product of $\delta$ 's. In doing so one has to introduce a factor accounting for the number of terms in each structure as

$$
\begin{aligned}
\delta_{\mu \mu} T_{\mu_{s-2}, \beta_{s-2}} & \rightsquigarrow \frac{s !}{2(s-2) !} \text { terms, } \\
\left(\delta^{\mu \mu}\right)^{m}\left(\delta^{\mu \beta}\right)^{s-2 m}\left(\delta^{\beta \beta}\right)^{m-1} & \rightsquigarrow \frac{s !}{2^{m} m !} \times \frac{(s-2) !}{2^{m-1}(m-1) !(s-2 m) !} \text { terms, }
\end{aligned}
$$

which implies

$$
\chi_{s}[n \vec{\theta}]=\frac{1}{s !} T^{[s]}+\sum_{m=1}^{\left\lfloor\frac{s}{2}\right\rfloor} \frac{(-1)^{m} 2^{m-1}(m-1) ![D+2(s-m-2)] ! !}{[(s-2) !]^{2}[D+2(s-2)] ! !} \delta_{\mu \mu}^{m} \delta_{\mu \beta}^{s-2 m} \delta_{\beta \beta}^{m-1} \delta^{\mu \mu} T^{\mu_{s-2}, \beta_{s-2}} .
$$

Taking into account the correct combinatorial factors one obtains

$$
\delta_{\mu \mu}^{m} \delta_{\mu \beta}^{s-2 m} \delta_{\beta \beta}^{m-1} \delta^{\mu \mu}=[D+2(s-m-1)] \delta_{\mu \mu}^{m-1} \delta_{\mu \beta}^{s-2 m} \delta_{\beta \beta}^{m-1}+2 m \delta_{\mu \mu}^{m} \delta_{\mu \beta}^{s-2 m-2} \delta_{\beta \beta}^{m},
$$

which then yields

$$
\begin{gathered}
\chi_{s}[n \vec{\theta}]=\frac{1}{s !} T^{[s]}+\left(\sum_{m=1}^{\left\lfloor\frac{s}{2}\right\rfloor} \frac{(-1)^{m} 2^{m-1}(m-1) ![D+2(s-m-1)] ! !}{[D+2(s-2)] ! !} \delta_{\mu \mu}^{m-1} \delta_{\mu \beta}^{s-2 m} \delta_{\beta \beta}^{m-1}\right. \\
\left.+\sum_{m=1}^{\left\lfloor\frac{s}{2}\right\rfloor-1} \frac{(-1)^{m} 2^{m} m ![D+2(s-m-2)] ! !}{[D+2(s-2)] ! !} \delta_{\mu \mu}^{m} \delta_{\mu \beta}^{s-2 m-2} \delta_{\beta \beta}^{m}\right) \frac{1}{[(s-2) !]^{2}} T^{\mu_{s-2}, \beta_{s-2}} .
\end{gathered}
$$

Shifting $m \rightarrow m+1$ in the upper sum one can see that both sums are identical apart from the overall sign and the lower extremum. Thus (A.16) boils down to

$$
\chi_{s}[n \vec{\theta}]=\frac{1}{s !} T^{[s]}-\frac{1}{[(s-2) !]^{2}} \delta_{\mu \beta}^{s-2} T^{\mu_{s-2}, \beta_{s-2}}=\frac{1}{s !} T^{[s]}-\frac{1}{(s-2) !} T^{[s-2]} .
$$

Now using (A.10) and (A.6) one obtains

$$
\chi_{s}[n \vec{\theta}]=\frac{1}{s !} T^{[s]}-\frac{1}{(s-2) !} T^{[s-2]}=h_{s}\left(\lambda_{1}, \lambda_{2}, \ldots, \lambda_{D}\right)-h_{s-2}\left(\lambda_{1}, \lambda_{2}, \ldots, \lambda_{D}\right),
$$

where $\lambda_{1}, \ldots, \lambda_{D}$ are the eigenvalues of $J^{n}$. (These eigenvalues are $e^{ \pm i n \theta_{j}}$ for $j=1, \ldots, r$, and one or two unit eigenvalues depending on whether $D$ is odd or even, respectively.) This leads to the desired result: since traces of powers of $J^{n}$ can be written as

$$
\operatorname{Tr}\left[\left(J^{n}\right)^{k}\right]=\lambda_{1}^{k}+\ldots+\lambda_{D}^{k}
$$

in terms of the eigenvalues of $J^{n}$, the complete homogeneous symmetric polynomials expressed as (A.5) exactly coincide with the combination (A.2), and equation (A.18) coincides with (A.1). 


\section{A.2 Symmetric polynomials and $\mathrm{SO}(D)$ characters}

In this part of the appendix we review the relation between complete homogeneous symmetric polynomials and characters of orthogonal groups. Most of the explicit proofs can be found in [85], chapter 24, to which we refer for details on the arguments exposed below. We will study separately the cases of odd and even $D$, and we let $r \equiv\lfloor(D-1) / 2\rfloor$, with $\theta_{1}, \ldots, \theta_{r}$ the non-vanishing angles appearing in the rotations (2.8).

Odd $\boldsymbol{D}$. We consider the Lie algebra $\mathfrak{s o}(D)=\mathfrak{s o}(2 r+1)$, with rank $r$. Choosing a basis of $\mathbb{C}^{2 r+1}$ such that the Lie algebra $\mathfrak{s o}(2 r+1)_{\mathbb{C}}$ can be written in terms of complex matrices, we may choose the Cartan subalgebra to be the subalgebra $\mathfrak{h}$ of $\mathfrak{s o}(2 r+1)_{\mathbb{C}}$ consisting of diagonal matrices. As a basis of $\mathfrak{h}$ we choose the matrices $H_{i}$ whose entries all vanish, except the $(i, i)$ and $(r+i, r+i)$ entries which are 1 and -1 , respectively (with $i=1, \ldots, r$ ). In our convention (2.7), the operator $H_{i}$ generates rotations in the plane $\left(x_{i}, y_{i}\right)$. Then, calling $L_{i}$ the elements of the dual basis (such that $\left\langle L_{i}, H_{j}\right\rangle=\delta_{i j}$ ), a dominant weight is one of the form $\lambda=\lambda_{1} L_{1}+\ldots \lambda_{r} L_{r} \equiv\left(\lambda_{1}, \ldots, \lambda_{r}\right)$ with $\lambda_{1} \geq \ldots \geq \lambda_{r} \geq 0$.

Let $\lambda$ be a dominant weight for $\mathfrak{s o}(2 r+1)$. According to formula (24.28) in [85], the character of the irreducible representation of $\mathfrak{s o}(2 r+1)$ with highest weight $\lambda$ is

$$
\chi_{\lambda}^{\mathrm{SO}(2 r+1)}\left[q_{1}, \ldots, q_{r}\right]=\operatorname{Tr}_{\lambda}\left[q_{1}^{H_{1}} \cdots q_{r}^{H_{r}}\right]=\frac{\left|q_{j}^{\lambda_{i}+r-i+\frac{1}{2}}-q_{j}^{-\left(\lambda_{i}+r-i+\frac{1}{2}\right)}\right|}{\left|q_{j}^{r-i+\frac{1}{2}}-q_{j}^{-\left(r-i+\frac{1}{2}\right)}\right|},
$$

where $q_{1}, \cdots q_{r}$ are arbitrary complex numbers, ${ }^{9} \operatorname{Tr}_{\lambda}$ denotes a trace taken in the space of the representation, and $\left|A_{i j}\right|$ denotes the determinant of the matrix $A$ with rows $i$ and columns $j$. This expression is a corollary of the Weyl character formula. Using proposition A.60 and Corollary A.46 of [85], it can be rewritten as

$$
\chi_{\lambda}^{\mathrm{SO}(2 r+1)}\left[q_{1}, \ldots, q_{r}\right]=\left|h_{\lambda_{i}-i+j}-h_{\lambda_{i}-i-j}\right|,
$$

where $h_{j}=h_{j}\left(q_{1}, \ldots, q_{n}, q_{1}^{-1}, \ldots, q_{n}^{-1}, 1\right)$ is a complete homogeneous symmetric polynomial of degree $j$ in $2 r+1$ variables. In particular, for a highest weight $\lambda_{s}=(s, 0, \ldots, 0)$ (where $s$ is a non-negative integer), the matrix appearing on the right-hand side of (A.21) is upper triangular, with the entry at $i=j=1$ given by $h_{s}-h_{s-2}$ and all other entries on the main diagonal equal to one. Accordingly, the determinant in (A.21) boils down to $h_{s}-h_{s-2}$ in that simple case. For the rotation (2.8) we may identify $q_{j}=e^{i n \theta_{j}}$, and we conclude that

$$
\chi_{\lambda_{s}}^{\mathrm{SO}(2 r+1)}[n \vec{\theta}]=\frac{\left|\sin \left[\left(\lambda_{i}+r-i+\frac{1}{2}\right) n \theta_{j}\right]\right|}{\left|\sin \left[\left(r-i+\frac{1}{2}\right) n \theta_{j}\right]\right|}=h_{s}\left(J^{n}\right)-h_{s-2}\left(J^{n}\right),
$$

where $\lambda_{i}=s \delta_{i 1}$. Thus for odd $D$ the difference of symmetric polynomials in (A.1) is just a character of $\mathrm{SO}(D)$.

\footnotetext{
${ }^{9}$ Eventually these numbers will be exponentials of angular potentials, so they are fugacities associated with the rotation generators $H_{i}$.
} 
Even $\boldsymbol{D}$. We now turn to the Lie algebra $\mathfrak{s o}(2 r+2)$, with rank $r+1$. As in the odd case we choose a basis of $\mathbb{C}^{2 r+2}$ such that we can write the Lie algebra $\mathfrak{s o}(2 r+2)$ in terms of complex matrices and the Cartan subalgebra is generated by $r+1$ diagonal matrices $H_{i}$ whose entries all vanish, except $\left(H_{i}\right)_{i i}=1$ and $\left(H_{i}\right)_{r+1+i, r+1+i}=-1$. We call $L_{i}$ the elements of the dual basis, and with these conventions a weight $\lambda=\lambda_{1} L_{1}+\ldots+\lambda_{r+1} L_{r+1} \equiv\left(\lambda_{1}, \ldots, \lambda_{r+1}\right)$ is dominant if $\lambda_{1} \geq \lambda_{2} \geq \ldots \geq \lambda_{r} \geq\left|\lambda_{r+1}\right|$.

Let $\lambda$ be a dominant weight for $\mathfrak{s o}(2 r+2)$. Then formula (24.40) in [85] gives the character of the associated highest-weight representation as

$$
\begin{aligned}
& \chi_{\lambda}^{\mathrm{SO}(2 r+2)}\left[q_{1}, \ldots, q_{r+1}\right]=\operatorname{Tr}_{\lambda}\left[q_{1}^{H_{1}} \cdots q_{r+1}^{H_{r+1}}\right] \\
& =\frac{\left|q_{j}^{\lambda_{i}+r+1-i}+q_{j}^{-\left(\lambda_{i}+r+1-i\right)}\right|+\left|q_{j}^{\lambda_{i}+r+1-i}-q_{j}^{-\left(\lambda_{i}+r+1-i\right)}\right|}{\left|q_{j}^{r+1-i}+q_{j}^{-(r+1-i)}\right|},
\end{aligned}
$$

where we use the same notations as in (A.20), except that now $i, j=1, \ldots, r+1$. Note that the second term in the numerator of this expression vanishes whenever $\lambda_{r+1}=0$ (because the $(r+1)^{\text {th }}$ row of the matrix $q_{j}^{\lambda_{i}+r+1-i}-q_{j}^{-\left(\lambda_{i}+r+1-i\right)}$ vanishes). Since this is the case that we will be interested in, we may safely forget about that second term from now on. Alternatively, for the mixed traces (2.20) that we need, we may take $q_{j}=e^{i n \theta_{j}}$ for $j=1, \ldots, r$ and $q_{r+1}=1$ without loss of generality, so that this second term vanishes again. Using proposition A.64 of [85], one can then rewrite (A.23) as

$$
\chi_{\lambda}^{\mathrm{SO}(2 r+2)}\left[q_{1}, \ldots, q_{r}, 1\right]=\left|h_{\lambda_{i}-i+j}-h_{\lambda_{i}-i-j}\right|,
$$

where $h_{j}=h_{j}\left(q_{1}, \ldots, q_{r}, 1, q_{1}^{-1}, \ldots, q_{r}^{-1}, 1\right)$. Finally, using the same arguments as for odd $D$, one easily verifies that the determinant on the right-hand side of (A.24) reduces once more to $h_{s}-h_{s-2}$ for a highest weight $\lambda_{s}=(s, 0, \ldots, 0)$. Writing again $q_{j}=e^{i n \theta_{j}}$, one concludes that, for even $D$,

$\chi_{\lambda_{s}}^{\mathrm{SO}(2 r+2)}\left[n \theta_{1}, \ldots, n \theta_{r}, n \theta_{r+1}=0\right]=\left.\frac{\left|\cos \left[\left(\lambda_{i}+r+1-i\right) n \theta_{j}\right]\right|}{\left|\cos \left[(r+1-i) n \theta_{j}\right]\right|}\right|_{\theta_{r+1}=0}=h_{s}\left(J^{n}\right)-h_{s-2}\left(J^{n}\right)$,

where $\lambda_{i}=s \delta_{i 1}$. This concludes the proof of (2.21). Note that, for non-vanishing $\theta_{r+1}$, the quotient of denominators in the middle of (A.25) is actually the character $\chi_{\lambda_{s}}^{\mathrm{SO}(2 r+2)}\left(n \theta_{1}, \ldots, n \theta_{r}, n \theta_{r+1}\right)$. This detail will be useful in appendix A.3.

\section{A.3 Differences of $\mathrm{SO}(D)$ characters}

In this part of the appendix we prove the following relations between characters of orthogonal groups:

$$
\begin{aligned}
\chi_{\lambda_{s}}^{\mathrm{SO}(2 r+1)}[\vec{\theta}]-\chi_{\lambda_{s-1}}^{\mathrm{SO}(2 r+1)}[\vec{\theta}] & =\chi_{\lambda_{s}}^{\mathrm{SO}(2 r)}[\vec{\theta}] \\
\chi_{\lambda_{s}}^{\mathrm{SO}(2 r)}[\vec{\theta}]-\chi_{\lambda_{s-1}}^{\mathrm{SO}(2 r)}[\vec{\theta}] & =\sum_{k=1}^{r} \mathcal{A}_{k}^{r}[\vec{\theta}] \chi_{\lambda_{s}}^{\mathrm{SO}(2 r-1)}\left[\theta_{1}, \ldots, \widehat{\theta_{k}}, \ldots, \theta_{r}\right] .
\end{aligned}
$$


Here $\vec{\theta}=\left(\theta_{1}, \ldots, \theta_{r}\right), \lambda_{s}$ is the weight with components $(s, 0, \ldots, 0)$ in the basis defined above equations (A.20) and (A.23), and the hat denotes omission of an argument, while the coefficients $\mathcal{A}_{k}^{r}$ are the quotients of determinants defined in (2.29). Note that, when one of the angles $\theta_{1}, \ldots, \theta_{r}$ vanishes, say $\theta_{\ell}=0$, then $\mathcal{A}_{k}^{r}=\delta_{k \ell}$ and relation (A.26b) reduces to

$$
\left.\chi_{\lambda_{s}}^{\mathrm{SO}(2 r)}[\vec{\theta}]\right|_{\theta_{\ell}=0}-\left.\chi_{\lambda_{s-1}}^{\mathrm{SO}(2 r)}[\vec{\theta}]\right|_{\theta_{\ell}=0}=\chi_{\lambda_{s}}^{\mathrm{SO}(2 r-1)}\left[\theta_{1}, \ldots, \widehat{\theta_{\ell}}, \ldots, \theta_{r}\right] .
$$

Proof of (A.26). We start by defining the matrices

$$
\left(A^{r}\right)_{i j}=\sin \left[\left(r-i+\frac{1}{2}\right) \theta_{j}\right], \quad\left(B^{r}\right)_{i j}=\cos \left[(r-i) \theta_{j}\right]
$$

so that in particular

$$
\mathcal{A}_{k}^{r}(\vec{\theta})=\frac{\left|B^{r}\right|_{\theta_{k}=0}}{\left|B^{r}\right|}
$$

We will also use the shorthand notation

$$
M^{r}\left[\theta_{k}\right] \equiv\left|M_{i j}\left(\theta_{1}, \ldots, \theta_{k-1}, \theta_{k+1}, \ldots, \theta_{r+1}\right)\right|
$$

to denote the determinant of the $r \times r$ matrix missing the angle $\theta_{k}$ of any of the matrices defined in (A.28). As a preliminary step towards the proof, we list the four following identities:

$$
\begin{aligned}
\frac{\left|A^{r}\right|}{\prod_{j=1}^{r} \sin \left(\theta_{j} / 2\right)} & =2^{r-1}\left|B^{r}\right| \\
\left|\cos \left[(r-i) \theta_{j}\right]\right| & =2^{\frac{(r-1)(r-2)}{2}} \prod_{\substack{1 \leq i<j \leq r \\
\text { i }}}^{r}\left(\cos \left(\theta_{i}\right)-\cos \left(\theta_{j}\right)\right) \\
\frac{\left|B^{r}\right|_{\theta_{k}=0}}{A^{r-1}\left[\theta_{k}\right]} & =2^{r-1}(-1)^{k+1} \prod_{\substack{j=1 \\
j \neq k}}^{r i n}\left(\theta_{j} / 2\right) \\
\left|B^{r}\right| & =\sum_{k=1}^{r}\left|B^{r}\right|_{\theta_{k}=0}
\end{aligned}
$$

Here (A.31a) can be proven by induction on $r$ upon expanding the determinant $\left|A^{r}(\vec{\theta})\right|$ along the first line of the matrix $A^{r}$. Property (A.31b) can be shown by observing that

$$
\cos \left[(r-i) \theta_{j}\right]=2^{r-i-1} \cos ^{r-i}\left(\theta_{j}\right)+\sum_{k=1}^{r-i-1} c_{k} \cos \left(k \theta_{j}\right)
$$

with some irrelevant real coefficients $c_{k}$, and that the contribution of the second term of this expression to the determinant $\left|\cos \left[(r-i) \theta_{j}\right]\right|$ vanishes by linear dependence. Equation (A.31c) then follows from (A.31a) and (A.31b), while property (A.31d) can again be proved by induction on $r$.

Equipped with eqs. (A.31), we can tackle the proof of (A.26). Equation (A.26a) is easy: using expression (A.22) for the character $\chi_{\lambda_{s}}^{\mathrm{SO}(2 r+1)}$, we can write the difference of 
characters on the left-hand side of (A.26a) as

$$
\chi_{\lambda_{s}}^{\mathrm{SO}(2 r+1)}-\chi_{\lambda_{s-1}}^{\mathrm{SO}(2 r+1)}=\frac{\sum_{k=1}^{r}(-1)^{k+1} 2 \cos \left[(s+r-1) \theta_{k}\right] \sin \left(\theta_{k} / 2\right) A^{r-1}\left[\theta_{k}\right]}{\left|A^{r}\right|} .
$$

Property (A.31a) then allows us to reduce this expression to the quotient of denominators appearing in the middle of eq. (A.25) (with the replacement of $r+1$ by $r$ and all angles non-zero), which is indeed the sought-for character $\chi_{\lambda_{s}}^{\mathrm{SO}(2 r)}[\vec{\theta}]$.

Equation (A.26b) requires more work. Using once more the expression in the middle of (A.25), we first rewrite the left-hand side of (A.26b) as

$$
\chi_{\lambda_{s}}^{\mathrm{SO}(2 r)}-\chi_{\lambda_{s-1}}^{\mathrm{SO}(2 r)}=\frac{\sum_{k=1}^{r}(-1)^{k+1}\left(-2 \sin \left[\left(s+r-\frac{3}{2}\right) \theta_{k}\right] \sin \left(\theta_{k} / 2\right) B^{r-1}\left[\theta_{k}\right]\right.}{\left|B^{r}\right|} .
$$

Let us now recover this expression as a combination of characters $\mathrm{SO}(2 r-1)$ : using formula (A.22) and the identities (A.31), one finds

$$
\begin{aligned}
& \sum_{k=1}^{r} \chi_{\lambda_{s}}^{\mathrm{SO}(2 r-1)}\left[\theta_{1}, \ldots, \widehat{\theta_{k}}, \ldots, \theta_{r}\right]\left|B^{r}\right|_{\theta_{k}=0} \\
& \stackrel{(\text { A.31c) }}{=} \sum_{k=1}^{r}(-1)^{k+1} 2^{r-1} \prod_{\substack{j=1 \\
j \neq k}}^{r} \sin \left(\theta_{j} / 2\right) \times \times\left[\sum_{j=1}^{k-1}(-1)^{j+1} \sin \left[\left(s+r-\frac{3}{2}\right) \theta_{j}\right] A^{r-2}\left[\theta_{j}, \theta_{k}\right]+\sum_{j=k+1}^{r}(-1)^{j} \sin \left[\left(s+r-\frac{3}{2}\right) \theta_{j}\right] A^{r-2}\left[\theta_{j}, \theta_{k}\right]\right] \\
& \stackrel{\text { (A.31a) }}{=} \sum_{k=1}^{r}(-1)^{k+1} 2^{2 r-4} \sin \left[\left(s+r-\frac{3}{2}\right) \theta_{k}\right] \sin \left(\theta_{k} / 2\right) \times \\
& \times\left[\sum_{j=1}^{k-1}(-1)^{j} B^{r-2}\left[\theta_{j}, \theta_{k}\right] \prod_{\substack{i=1 \\
i \notin\{j, k\}}}^{r} \sin ^{2}\left(\theta_{i} / 2\right)+\sum_{j=k+1}^{r}(-1)^{j+1} B^{r-2}\left[\theta_{j}, \theta_{k}\right] \prod_{i=1}^{r} \sin ^{2}\left(\theta_{i} / 2\right)\right] \\
& \stackrel{\text { (A.31b) }}{=} \sum_{k=1}^{r}(-1)^{k+1}(-2) \sin \left[\left(s+r-\frac{3}{2}\right) \theta_{k}\right] \sin \left(\theta_{k} / 2\right)\left[\left.\sum_{j=1}^{k-1}(-1)^{j} B^{r-1}\left[\theta_{k}\right]\right|_{\theta_{j}=0}+\left.\sum_{j=k+1}^{r} B^{r-1}\left[\theta_{k}\right]\right|_{\theta_{j}=0}\right] \\
& \stackrel{\text { (A.31d) }}{=} \sum_{k=1}^{r}(-1)^{k+1}(-2) \sin \left[\left(s+r-\frac{3}{2}\right) \theta_{k}\right] \sin \left(\theta_{k} / 2\right) B^{r-1}\left[\theta_{k}\right] .
\end{aligned}
$$

This coincides with the numerator of the right-hand side of (A.34), so identity (A.26b) follows with $\mathcal{A}_{k}^{r}$ given by (A.29).

\section{A.4 From $\mathrm{SO}(D)$ to $\mathrm{SO}(D-1)$}

In this appendix we prove relation (2.32) between characters of $\mathrm{SO}(D)$ and $\mathrm{SO}(D-1)$ :

\section{Lemma.}

$$
\chi_{\lambda_{s}}^{\mathrm{SO}(2 r+1)}\left[\theta_{1}, \ldots, \theta_{r}\right]=\sum_{j=0}^{s} \chi_{\lambda_{j}}^{\mathrm{SO}(2 r)}\left(\theta_{1}, \ldots, \theta_{r}\right),
$$




$$
\chi_{\lambda_{s}}^{\mathrm{SO}(2 r)}\left[\theta_{1}, \ldots, \theta_{r}\right]=\sum_{j=0}^{s} \sum_{k=1}^{r} \mathcal{A}_{k}^{r}(\vec{\theta}) \chi_{\lambda_{j}}^{\mathrm{SO}(2 r-1)}\left[\theta_{1}, \ldots, \widehat{\theta_{k}}, \ldots, \theta_{r}\right] .
$$

Here $\lambda_{j}$ is the weight $(j, 0, \ldots, 0)$ as explained above (2.21) or below (A.21), and $\mathcal{A}_{k}^{r}(\vec{\theta})$ is the quotient (2.29) or (A.29). Since the proofs of these two identities are very similar, we will only display the proof of (A.36a).

Proof of (A.36a). Eq. (A.36a) can be written as

$$
\frac{\sum_{k=1}^{r}(-1)^{k+1} \sin \left[\left(s+r-\frac{1}{2}\right) \theta_{k}\right] A^{r-1}\left[\theta_{k}\right]}{\left|A^{r}\right|}(\mathrm{A} .31 \mathrm{a}) \underset{\&}{=}(\mathrm{A} .31 \mathrm{c}) \sum_{j=0}^{s} \frac{\sum_{k=1}^{r}(-1)^{k+1} \cos \left[(j+r-1) \theta_{k}\right] B^{r-1}\left[\theta_{k}\right]}{\left|B^{r}\right|},
$$

where we used formulas (A.22) and (A.25) for the characters, as well as the definition (A.28) of $A^{r}$ and $B^{r}$. One can then use identities (A.31a) and (A.31c) to match the right-hand side of this expression with the left-hand side, proving the desired identity.

\section{B From mixed traces to fermionic characters}

\section{B.1 Mixed traces and symmetric polynomials}

Our goal here is to prove the first equality of (2.48), following the same method as in appendix A.1 for the bosonic case. First, using the definition (2.43) of $U$ and the contraction rules (A.11), one can write (2.47) as

$$
\begin{aligned}
\chi_{s}^{(F)}[n \vec{\theta}] & =\left[\frac{1}{s !} T^{[s]}+\sum_{m=1}^{\left\lfloor\frac{s}{2}\right\rfloor} \frac{(-1)^{m}[D+2(s-m-1)] ! !}{2^{m-1} m !(s-2 m) ![D+2(s-1)] ! !}\left(\delta^{\mu \mu}\right)^{m}\left(\delta^{\mu \beta}\right)^{s-2 m}\left(\delta^{\beta \beta}\right)^{m-1} \delta_{\mu \mu} T_{\mu_{s-2}, \beta_{s-2}}\right] \operatorname{Tr}\left[U^{n}\right] \\
& \left.+\sum_{m=0}^{\left\lfloor\frac{s-1}{2}\right\rfloor} \frac{(-1)^{m+1}[D+2(s-m-2)] ! !}{2^{m} m !(s-2 m-1) ![D+2(s-1)] ! !} \operatorname{Tr}\left[T_{\mu_{s-1}, \beta_{s-1}} \gamma_{\mu} \gamma^{\mu}\left(\delta^{\mu \mu}\right)^{m}\left(\delta^{\mu \beta}\right)^{s-2 m-1}\left(\delta^{\beta \beta}\right)^{m} U^{n}\right], \quad \text { B. }\right)
\end{aligned}
$$

where $T^{[s]}$ is the notation (A.10). In the first term of this expression, we shift the symmetrisation on the $\delta$ 's i.e. we exchange upper and lower indices while taking into account the change in multiplicities of the terms involved; in all other terms, we compute one contraction with $\delta^{\beta \beta}$. Eq. (B.1) then simplifies to

$$
\begin{aligned}
& \chi_{s}^{(F)}[n \vec{\theta}]=\frac{1}{s !} T^{[s]} \operatorname{Tr}\left[U^{n}\right]-\frac{1}{[(s-1) !]^{2}[D+2(s-1)]} \operatorname{Tr}\left[T^{\mu_{s-1}, \beta_{s-1}} \gamma_{\mu} \gamma^{\mu} \delta_{\mu \beta}^{s-1} U^{n}\right] \\
& +\sum_{m=1}^{\left\lfloor\frac{s}{2}\right\rfloor}\left[\frac{(-1)^{m} 2^{m-1}(m-1) ![D+2(s-m-1)] ! !}{[(s-2) !]^{2}[D+2(s-1)] ! !} T^{\mu_{s-2}, \beta_{s-2}} \delta^{\mu \mu} \delta_{\mu \mu}^{m} \delta_{\mu \beta}^{s-2 m} \delta_{\beta \beta}^{m-1} \operatorname{Tr}\left[U^{n}\right]\right. \\
& \left.+\frac{(-1)^{m+1} 2 m-1(m-1) ![D+2(s-m-2)] ! !}{[(s-2) !]^{2}[D+2(s-1)] ! !} \operatorname{Tr}\left[T^{\mu_{s-2}, \beta_{s-2}} \delta^{\mu \mu} \delta_{\mu \mu}^{m} \delta_{\mu \beta}^{s-2 m-1} \delta_{\beta \beta}^{m} \gamma_{\mu} \gamma_{\beta} U^{n}\right]\right] .
\end{aligned}
$$

The $\gamma$ traces and mixed traces can now be evaluated using

$$
\begin{gathered}
\gamma^{\mu} \gamma_{\mu} \delta_{\mu \beta}^{s-1}=[D+2(s-1)] \delta_{\mu \beta}^{s-1}-\gamma_{\mu} \gamma_{\beta} \delta_{\mu \beta}^{s-2}, \\
\delta^{\mu \mu} \delta_{\mu \mu}^{m} \delta_{\mu \beta}^{s-2 m} \delta_{\beta \beta}^{m-1}=[D+2(s-m-1)] \delta_{\mu \mu}^{m-1} \delta_{\mu \beta}^{s-2 m} \delta_{\beta \beta}^{m-1}
\end{gathered}
$$




$$
\begin{aligned}
& +2 m \delta_{\mu \mu}^{m} \delta_{\mu \beta}^{s-2 m-2} \delta_{\beta \beta}^{m}, \\
\delta^{\mu \mu} \delta_{\mu \mu}^{m} \delta_{\mu \beta}^{s-2 m-1} \delta_{\beta \beta}^{m-1} \gamma_{\mu} \gamma_{\beta}= & {[D+2(s-m-1)] \delta_{\mu \mu}^{m-1} \delta_{\mu \beta}^{s-2 m-1} \delta_{\beta \beta}^{m-1} \gamma_{\mu} \gamma_{\beta} } \\
& +4 m \delta_{\mu \mu}^{m} \delta_{\mu \beta}^{s-2 m-2} \delta_{\beta \beta}^{m}+2 m \delta_{\mu \mu}^{m} \delta_{\mu \beta}^{s-2 m-3} \delta_{\beta \beta}^{m} \gamma_{\mu} \gamma_{\beta},
\end{aligned}
$$

which yields

$$
\begin{aligned}
\chi_{s}^{(F)}[n \vec{\theta}]= & {\left[\frac{1}{s !} T^{[s]}-\frac{1}{(s-1) !} T^{[s-1]}\right] \operatorname{Tr}\left[U^{n}\right]+\frac{1}{[(s-1) !]^{2}[D+2(s-1)]} \operatorname{Tr}\left[T^{\mu_{s-1}, \beta_{s-1}} \delta_{\mu \beta}^{s-2} \gamma_{\mu} \gamma_{\beta} U^{n}\right] } \\
& -\frac{D+2(s-2)}{[(s-2) !]^{2}[D+2(s-1)]} T^{\mu_{s-2}, \beta_{s-2}} \delta_{\mu \beta}^{s-2} \operatorname{Tr}\left[U^{n}\right] \\
& +\frac{1}{[(s-2) !]^{2}[D+2(s-1)]} \operatorname{Tr}\left[T^{\mu_{s-2}, \beta_{s-2}} \delta_{\mu \beta}^{s-3} \gamma_{\mu} \gamma_{\beta} U^{n}\right] .
\end{aligned}
$$

Using (A.18) and the definition (2.43) of $U$, together with some careful counting, one verifies that this expression matches $\left[h_{s}\left(J^{n}\right)-h_{s-1}\left(J^{n}\right)\right] \operatorname{Tr}\left[U^{n}\right]$, which was to be proven.

\section{B.2 Symmetric polynomials and $\mathrm{SO}(D)$ characters}

In this part of the appendix we prove the second equality in (2.48), following essentially the same steps as in appendix A.2. We refer again to [85] for details, and we write the components of weights in the dual basis of the Cartan subalgebra described above (A.20) and (A.23). We will consider separately odd and even space-time dimensions.

Odd $\boldsymbol{D}$. The character of a half-spin representation of $\mathfrak{s o}(2 r+1)$ with a dominant highest weight $\lambda=\left(\lambda_{1}+\frac{1}{2}, \lambda_{2}+\frac{1}{2}, \ldots, \lambda_{r}+\frac{1}{2}\right)$ is $[86$, p.258f $]$

$$
\chi_{\lambda}^{\mathrm{SO}(2 r+1)}\left[\theta_{1}, \ldots, \theta_{r}\right]=\frac{\left|\sin \left[\left(\lambda_{i}+r-i+1\right) \theta_{j}\right]\right|}{\left|\sin \left[\left(r-i+\frac{1}{2}\right) \theta_{j}\right]\right|}=\left(\prod_{i=1}^{r} 2 \cos \left(\frac{\theta_{i}}{2}\right)\right) \frac{\left|\sin \left[\left(\lambda_{i}+r-i+1\right) \theta_{j}\right]\right|}{\left|\sin \left[(r-i+1) \theta_{j}\right]\right|} .
$$

Owing to expression (2.45) for the trace of $U^{n}$, the second equality in (2.48) is equivalent to

$$
h_{s}(J)-h_{s-1}(J)=\frac{\left|\sin \left[\left(\lambda_{i}+r-i+1\right) \theta_{j}\right]\right|}{\left|\sin \left[(r-i+1) \theta_{j}\right]\right|}
$$

for $\lambda_{i}=s \delta_{i 1}$. To prove this, consider the difference of the bosonic character (A.22) and the right-hand side of (B.6):

$$
\frac{\left|\sin \left[\left(\lambda_{i}+r-i+\frac{1}{2}\right) \theta_{j}\right]\right|}{\left|\sin \left[\left(r-i+\frac{1}{2}\right) \theta_{j}\right]\right|}-\frac{\left|\sin \left[\left(\lambda_{i}+r-i+1\right) \theta_{j}\right]\right|}{\left|\sin \left[(r-i+1) \theta_{j}\right]\right|} .
$$

Introducing the notation

$$
\left(\mathcal{A}^{r}\right)_{i j}=2 \sin \left[(r-i+1) \theta_{j}\right], \quad\left(\mathcal{B}^{r}\right)_{i j}=2 \cos \left[\left(r-i+\frac{1}{2}\right) \theta_{j}\right]
$$

and in terms of (A.28), this difference can be written as

$$
\frac{\sum_{k=1}^{r}(-1)^{k+1} \sin \left[\left(s+r-\frac{1}{2}\right) \theta_{k}\right] A^{r-1}\left[\theta_{k}\right]}{\left|A^{r}\right|}-\frac{\sum_{k=1}^{r}(-1)^{k+1} 2 \sin \left[(s+r) \theta_{k}\right] \mathcal{A}^{r-1}\left[\theta_{k}\right]}{\left|\mathcal{A}^{r}\right|}
$$


upon expanding the determinants along the first row. Now it turns out that ${ }^{10}$

$$
2^{r}\left|A^{r}\right| \prod_{i=1}^{r} 2 \cos \left(\theta_{i} / 2\right)=\left|\mathcal{A}^{r}\right|, \quad 2^{r-1}\left|B^{r}\right| \prod_{i=1}^{r} 2 \cos \left(\theta_{i} / 2\right)=\left|\mathcal{B}^{r}\right|,
$$

and plugging this property in (B.9) one sees that (B.7) is just $h_{s-1}(J)-h_{s-2}(J)$. Since the first term of (B.7) equals $h_{s}(J)-h_{s-2}(J)$ by virtue of (A.22), this proves (B.6).

Even $\boldsymbol{D}$. The character of an irreducible representation of $\mathfrak{s o}(2 r+2)$ with (dominant) highest-weight $\lambda=\left(\lambda_{1}+1 / 2, \ldots, \lambda_{r+1}+1 / 2\right)$ can be written as [86, p.258-259]

$$
\chi_{\lambda}^{\mathrm{SO}(2 r+2)}\left[\theta_{1}, \ldots, \theta_{r}\right]=\frac{\left|\cos \left[\left(\lambda_{i}+r-i+\frac{3}{2}\right) \theta_{j}\right]\right|}{\left|\cos \left[(r-i+1) \theta_{j}\right]\right|}=\prod_{i=1}^{r+1} 2 \cos \left(\frac{\theta_{i}}{2}\right) \frac{\left|\cos \left[\left(\lambda_{i}+r-i+\frac{3}{2}\right) \theta_{j}\right]\right|}{\left|\cos \left[\left(r-i+\frac{3}{2}\right) \theta_{j}\right]\right|}
$$

where we are including the possibility of a non-zero angles $\theta_{r+1}$ (while in (2.48) we take $\left.\theta_{r+1}=0\right)$. Taking into account $(2.45)$, proving the second equality in (2.48) amounts to showing that

$$
h_{s}(J)-h_{s-1}(J)=\left.\frac{\left|\cos \left[\left(\lambda_{i}+r-i+\frac{3}{2}\right) \theta_{j}\right]\right|}{\left|\cos \left[\left(r-i+\frac{3}{2}\right) \theta_{j}\right]\right|}\right|_{\theta_{r+1}=0}
$$

for $\lambda_{i}=s \delta_{i 1}$. To prove this we proceed as in the odd-dimensional case: the difference of the bosonic character (A.25) and the right-hand side of (B.12),

$$
\left.\frac{\left|\cos \left[\left(\lambda_{i}+r-i+1\right) \theta_{j}\right]\right|}{\left|\cos \left[(r-i+1) \theta_{j}\right]\right|}\right|_{\theta_{r+1}=0}-\left.\frac{\left|\cos \left[\left(\lambda_{i}+r-i+\frac{3}{2}\right) \theta_{j}\right]\right|}{\left|\cos \left[\left(r-i+\frac{3}{2}\right) \theta_{j}\right]\right|}\right|_{\theta_{r+1}=0},
$$

can be written as

$$
\left[\frac{\sum_{k=1}^{r+1}(-1)^{k+1} \cos \left[(s+r) \theta_{k}\right] B^{r}\left[\theta_{k}\right]}{\left|B^{r+1}\right|}-\frac{\sum_{k=1}^{r+1}(-1)^{k+1} 2 \cos \left[\left(s+r+\frac{1}{2}\right) \theta_{k}\right] \mathcal{B}^{r}\left[\theta_{k}\right]}{\left|\mathcal{B}^{r+1}\right|}\right]_{\theta_{r+1}=0}
$$

upon expanding the determinants along the first row and using the notation (A.28)-(B.8). One can then verify that this reduces to $h_{s-1}(J)-h_{s-2}(J)$ by the same argument as in the odd-dimensional case. By virtue of the second equality in (A.25), this proves (B.12).

Open Access. This article is distributed under the terms of the Creative Commons Attribution License (CC-BY 4.0), which permits any use, distribution and reproduction in any medium, provided the original author(s) and source are credited.

\section{References}

[1] M.A. Vasiliev, Nonlinear equations for symmetric massless higher spin fields in (A)d $S_{d}$, Phys. Lett. B 567 (2003) 139 [hep-th/0304049] [INSPIRE].

\footnotetext{
${ }^{10}$ See e.g. [86, p.259].
} 
[2] X. Bekaert, N. Boulanger and P. Sundell, How higher-spin gravity surpasses the spin two barrier: no-go theorems versus yes-go examples, Rev. Mod. Phys. 84 (2012) 987 [arXiv: 1007.0435] [INSPIRE].

[3] A. Sagnotti, Notes on strings and higher spins, J. Phys. A 46 (2013) 214006 [arXiv:1112.4285] [INSPIRE].

[4] M.R. Gaberdiel, R. Gopakumar and A. Saha, Quantum $W$-symmetry in $A d S_{3}$, JHEP 02 (2011) 004 [arXiv: 1009.6087] [INSPIRE].

[5] M.R. Gaberdiel, R. Gopakumar, T. Hartman and S. Raju, Partition functions of holographic minimal models, JHEP 08 (2011) 077 [arXiv: 1106.1897] [INSPIRE].

[6] T. Creutzig, Y. Hikida and P.B. Ronne, Higher spin AdS supergravity and its dual CFT, JHEP 02 (2012) 109 [arXiv:1111.2139] [INSPIRE].

[7] M.R. Gaberdiel and R. Gopakumar, Minimal model holography, J. Phys. A 46 (2013) 214002 [arXiv:1207.6697] [inSPIRE].

[8] S. Giombi and I.R. Klebanov, One loop tests of higher spin AdS/CFT, JHEP 12 (2013) 068 [arXiv: 1308.2337] [INSPIRE].

[9] S. Giombi, I.R. Klebanov and B.R. Safdi, Higher spin $A d S_{d+1} / C F T_{d}$ at one loop, Phys. Rev. D 89 (2014) 084004 [arXiv: 1401.0825] [inSPIRE].

[10] S. Giombi, I.R. Klebanov and A.A. Tseytlin, Partition functions and Casimir energies in higher spin $A d S_{d+1} / C F T_{d}$, Phys. Rev. D 90 (2014) 024048 [arXiv:1402.5396] [INSPIRE].

[11] M. Beccaria and A.A. Tseytlin, Higher spins in $A d S_{5}$ at one loop: vacuum energy, boundary conformal anomalies and AdS/CFT, JHEP 11 (2014) 114 [arXiv:1410.3273] [INSPIRE].

[12] M. Beccaria and A.A. Tseytlin, On higher spin partition functions, J. Phys. A 48 (2015) 275401 [arXiv:1503.08143] [INSPIRE].

[13] I.R. Klebanov and A.M. Polyakov, AdS dual of the critical $O(N)$ vector model, Phys. Lett. B 550 (2002) 213 [hep-th/0210114] [INSPIRE].

[14] A.A. Tseytlin, On partition function and Weyl anomaly of conformal higher spin fields, Nucl. Phys. B 877 (2013) 598 [arXiv: 1309.0785] [INSPIRE].

[15] M. Beccaria, X. Bekaert and A.A. Tseytlin, Partition function of free conformal higher spin theory, JHEP 08 (2014) 113 [arXiv: 1406.3542] [INSPIRE].

[16] T. Basile, X. Bekaert and N. Boulanger, Flato-Fronsdal theorem for higher-order singletons, JHEP 11 (2014) 131 [arXiv:1410.7668] [INSPIRE].

[17] M.P. Blencowe, A consistent interacting massless higher spin field theory in $D=(2+1)$, Class. Quant. Grav. 6 (1989) 443 [INSPIRE].

[18] A. Campoleoni, Higher spins in $D=2+1$, Subnucl. Ser. 49 (2013) 385 [arXiv:1110.5841] [INSPIRE].

[19] C. Fronsdal, Massless fields with integer spin, Phys. Rev. D 18 (1978) 3624 [inSPIRE].

[20] J. Fang and C. Fronsdal, Massless fields with half integral spin, Phys. Rev. D 18 (1978) 3630 [INSPIRE].

[21] A. Campoleoni and M. Henneaux, Asymptotic symmetries of three-dimensional higher-spin gravity: the metric approach, JHEP 03 (2015) 143 [arXiv: 1412.6774] [INSPIRE]. 
[22] J.D. Brown and M. Henneaux, Central charges in the canonical realization of asymptotic symmetries: an example from three-dimensional gravity,

Commun. Math. Phys. 104 (1986) 207 [INSPIRE].

[23] M. Henneaux and S.-J. Rey, Nonlinear $W_{\infty}$ as asymptotic symmetry of three-dimensional higher spin anti-de Sitter gravity, JHEP 12 (2010) 007 [arXiv:1008.4579] [INSPIRE].

[24] A. Campoleoni, S. Fredenhagen, S. Pfenninger and S. Theisen, Asymptotic symmetries of three-dimensional gravity coupled to higher-spin fields, JHEP 11 (2010) 007 [arXiv: 1008.4744] [INSPIRE].

[25] M.R. Gaberdiel and T. Hartman, Symmetries of holographic minimal models, JHEP 05 (2011) 031 [arXiv:1101.2910] [INSPIRE].

[26] A. Campoleoni, S. Fredenhagen and S. Pfenninger, Asymptotic $W$-symmetries in three-dimensional higher-spin gauge theories, JHEP 09 (2011) 113 [arXiv:1107.0290] [INSPIRE].

[27] A. Ashtekar, J. Bicak and B.G. Schmidt, Asymptotic structure of symmetry reduced general relativity, Phys. Rev. D 55 (1997) 669 [gr-qc/9608042] [INSPIRE].

[28] G. Barnich and G. Compere, Classical central extension for asymptotic symmetries at null infinity in three spacetime dimensions, Class. Quant. Grav. 24 (2007) F15 [gr-qc/0610130] [INSPIRE].

[29] G. Barnich and C. Troessaert, Aspects of the BMS/CFT correspondence, JHEP 05 (2010) 062 [arXiv: 1001.1541] [INSPIRE].

[30] H. Afshar, A. Bagchi, R. Fareghbal, D. Grumiller and J. Rosseel, Spin-3 gravity in three-dimensional flat space, Phys. Rev. Lett. 111 (2013) 121603 [arXiv:1307.4768] [INSPIRE].

[31] H.A. Gonzalez, J. Matulich, M. Pino and R. Troncoso, Asymptotically flat spacetimes in three-dimensional higher spin gravity, JHEP 09 (2013) 016 [arXiv:1307.5651] [INSPIRE].

[32] O. Fuentealba, J. Matulich and R. Troncoso, Asymptotically flat structure of hypergravity in three spacetime dimensions, JHEP 10 (2015) 009 [arXiv: 1508.04663] [INSPIRE].

[33] P. Bouwknegt and K. Schoutens, $W$ symmetry in conformal field theory, Phys. Rept. 223 (1993) 183 [hep-th/9210010] [INSPIRE].

[34] S. Giombi, A. Maloney and X. Yin, One-loop partition functions of $3 D$ gravity, JHEP 08 (2008) 007 [arXiv:0804.1773] [INSPIRE].

[35] G. Barnich, H.A. Gonzalez, A. Maloney and B. Oblak, One-loop partition function of three-dimensional flat gravity, JHEP 04 (2015) 178 [arXiv:1502.06185] [INSPIRE].

[36] J.R. David, M.R. Gaberdiel and R. Gopakumar, The heat kernel on $A d S_{3}$ and its applications, JHEP 04 (2010) 125 [arXiv:0911.5085] [INSPIRE].

[37] R. Gopakumar, R.K. Gupta and S. Lal, The heat kernel on AdS, JHEP 11 (2011) 010 [arXiv: 1103.3627] [INSPIRE].

[38] D. Grumiller, M. Riegler and J. Rosseel, Unitarity in three-dimensional flat space higher spin theories, JHEP 07 (2014) 015 [arXiv: 1403.5297] [INSPIRE].

[39] D.V. Vassilevich, Heat kernel expansion: user's manual, Phys. Rept. 388 (2003) 279 [hep-th/0306138] [INSPIRE]. 
[40] L.P.S. Singh and C.R. Hagen, Lagrangian formulation for arbitrary spin. 1. The boson case, Phys. Rev. D 9 (1974) 898 [inSPIRE].

[41] Yu. M. Zinoviev, On massive high spin particles in AdS, hep-th/0108192 [INSPIRE].

[42] R. Rahman, Higher spin theory — part I, PoS (Modave VIII) 004 [arXiv:1307.3199] [INSPIRE].

[43] S. Prohazka, J. Salzer and F. Schöller, One-loop partition function of three-dimensional flat topologically massive gravity, in preparation.

[44] G.W. Gibbons, M.J. Perry and C.N. Pope, Partition functions, the Bekenstein bound and temperature inversion in anti-de Sitter space and its conformal boundary, Phys. Rev. D 74 (2006) 084009 [hep-th/0606186] [INSPIRE].

[45] A. Bagchi, S. Detournay, D. Grumiller and J. Simon, Cosmic evolution from phase transition of three-dimensional flat space, Phys. Rev. Lett. 111 (2013) 181301 [arXiv:1305.2919] [INSPIRE].

[46] L.P.S. Singh and C.R. Hagen, Lagrangian formulation for arbitrary spin. 2. The fermion case, Phys. Rev. D 9 (1974) 910 [INSPIRE].

[47] R.R. Metsaev, Gauge invariant formulation of massive totally symmetric fermionic fields in (A)dS space, Phys. Lett. B 643 (2006) 205 [hep-th/0609029] [INSPIRE].

[48] G. Barnich and B. Oblak, Notes on the BMS group in three dimensions: I. Induced representations, JHEP 06 (2014) 129 [arXiv: 1403.5803] [INSPIRE].

[49] B. Oblak, Characters of the BMS group in three dimensions, Commun. Math. Phys. 340 (2015) 413 [arXiv:1502.03108] [INSPIRE].

[50] E.P. Wigner, On unitary representations of the inhomogeneous Lorentz group, Annals Math. 40 (1939) 149 [INSPIRE].

[51] G.W. Mackey, On induced representations of groups, Amer. J. Math. 73 (1951) 576.

[52] G.W. Mackey, Induced representations of locally compact groups I, Ann. Math. 55 (1952) 101.

[53] A. Kirillov, Elements of the theory of representations, Grundlehren der mathematischen Wissenschaften 220, Springer-Verlag, Germany (1976).

[54] H. Joos and R. Schrader, On the primitive characters of the Poincaré group, Commun. Math. Phys. 7 (1968) 21.

[55] H.X. Nghiem, Harmonic analysis on the Poincaré group. I. Generalized matrix elements, Commun. Math. Phys. 12 (1969) 331.

[56] A. Garbarz and M. Leston, Quantization of $B M S_{3}$ orbits: a perturbative approach, arXiv: 1507.00339 [INSPIRE].

[57] F.A. Dolan, Character formulae and partition functions in higher dimensional conformal field theory, J. Math. Phys. 47 (2006) 062303 [hep-th/0508031] [INSPIRE].

[58] G. Barnich and B. Oblak, Notes on the BMS group in three dimensions: II. Coadjoint representation, JHEP 03 (2015) 033 [arXiv:1502.00010] [INSPIRE].

[59] E. Witten, Coadjoint orbits of the Virasoro group, Commun. Math. Phys. 114 (1988) 1 [INSPIRE]. 
[60] J. Balog, L. Feher and L. Palla, Coadjoint orbits of the Virasoro algebra and the global Liouville equation, Int. J. Mod. Phys. A 13 (1998) 315 [hep-th/9703045] [INSPIRE].

[61] G. Barnich and C. Troessaert, Supertranslations call for superrotations, PoS (CNCFG2010) 010 [arXiv:1102.4632] [INSPIRE].

[62] E. İnönü and E.P. Wigner, On the contraction of groups and their represenations, Proc. Nat. Acad. Sci. 39 (1953) 510 [inSPIRE].

[63] A. Campoleoni, H.A. Gonzalez, B. Oblak and M. Riegler, BMS modules in three dimensions, arXiv: 1603.03812 [INSPIRE].

[64] A. Bagchi, R. Gopakumar, I. Mandal and A. Miwa, GCA in 2d, JHEP 08 (2010) 004 [arXiv:0912.1090] [INSPIRE].

[65] J. Balog, L. Feher, L. O'Raifeartaigh, P. Forgacs and A. Wipf, Toda theory and W algebra from a gauged WZNW point of view, Annals Phys. 203 (1990) 76 [INSPIRE].

[66] B.A. Khesin and B.Z. Shapiro, Nondegenerate curves on $S^{2}$ and orbit classification of the Zamolodchikov algebra, Commun. Math. Phys. 145 (1992) 357 [InSPIRE].

[67] Z. Bajnok and D. Nogradi, Geometry of $W$ algebras from the affine Lie algebra point of view, J. Phys. A 34 (2001) 4811 [hep-th/0012190] [INSPIRE].

[68] E. Shavgulidze, An example of a measure quasi-invariant under the action of the diffeomorphism group of the circle, Funct. Anal. Appl. 12 (1978) 203 [Funkts. Anal. Prilozh. 12 (1978) 55].

[69] G. Barnich, L. Donnay, J. Matulich and R. Troncoso, Asymptotic symmetries and dynamics of three-dimensional flat supergravity, JHEP 08 (2014) 071 [arXiv:1407.4275] [INSPIRE].

[70] G. Barnich, L. Donnay, J. Matulich and R. Troncoso, Super-BMS 3 invariant boundary theory from three-dimensional flat supergravity, arXiv:1510.08824 [INSPIRE].

[71] O. Fuentealba, J. Matulich and R. Troncoso, Extension of the Poincaré group with half-integer spin generators: hypergravity and beyond, JHEP 09 (2015) 003 [arXiv: 1505.06173] [INSPIRE].

[72] I. Mandal, Supersymmetric extension of GCA in 2d, JHEP 11 (2010) 018 [arXiv:1003.0209] [INSPIRE].

[73] C. Carmeli, G. Cassinelli, A. Toigo and V.S. Varadarajan, Unitary representations of super Lie groups and applications to the classification and multiplet structure of super particles, Commun. Math. Phys. 263 (2006) 217 [Erratum ibid. 307 (2011) 565] [hep-th/0501061] [INSPIRE].

[74] C. Carmeli, Super Lie groups: structure and representations, Ph.D. thesis, available on http://www.ge.infn.it/ carmeli/publication/publication.html, Università degli Studi Di Genova, Genova Italy (2006).

[75] P. Deligne and I. Study, Quantum fields and strings: a course for mathematicians, volume 2, American Mathematical Soc., U.S.A. (1999).

[76] J. Dai and D. Pickrell, The orbit method and the Virasoro extension of Diff+(S1) - I. Orbital integrals, J. Geom. Phys. 44 (2003) 623.

[77] L. Guieu and C. Roger, L'algèbre et le groupe de Virasoro (in French), Publications du CRM, Université de Montréal, Montréal Canada (2007). 
[78] O. Coussaert and M. Henneaux, Supersymmetry of the $(2+1)$ black holes, Phys. Rev. Lett. 72 (1994) 183 [hep-th/9310194] [INSPIRE].

[79] P. Schuster and N. Toro, On the theory of continuous-spin particles: wavefunctions and soft-factor scattering amplitudes, JHEP 09 (2013) 104 [arXiv:1302.1198] [INSPIRE].

[80] P. Schuster and N. Toro, Continuous-spin particle field theory with helicity correspondence, Phys. Rev. D 91 (2015) 025023 [arXiv: 1404.0675] [INSPIRE].

[81] A. Campoleoni, D. Francia, J. Mourad and A. Sagnotti, Unconstrained higher spins of mixed symmetry. I. Bose fields, Nucl. Phys. B 815 (2009) 289 [arXiv:0810.4350] [INSPIRE].

[82] A. Campoleoni, D. Francia, J. Mourad and A. Sagnotti, Unconstrained higher spins of mixed symmetry. II. Fermi fields, Nucl. Phys. B 828 (2010) 405 [arXiv: 0904.4447] [inSPIRE].

[83] R.K. Gupta and S. Lal, Partition functions for higher-spin theories in AdS, JHEP 07 (2012) 071 [arXiv:1205.1130] [INSPIRE].

[84] I. Macdonald, Symmetric functions and Hall polynomials, second edition, Oxford Mathematical Monographs, Oxford Science Publications, Oxford U.K. (1995).

[85] W. Fulton and J. Harris, Representation theory: a first course, Springer Science \& Business Media, Germany (1991).

[86] D.E. Littlewood, The theory of group characters and matrix representations of groups, American Mathematical Soc., U.S.A. (1950). 